\title{
Limit laws and automorphism groups of random nonrigid structures
}

\author{
OVE AHLMAN \\ VERA KOPONEN
}

\begin{abstract}
A systematic study is made, for an arbitrary finite relational language with at least one symbol of arity at least 2 , of classes of nonrigid finite structures. The well known results that almost all finite structures are rigid and that the class of finite structures has a zero-one law are, in the present context, the first layer in a hierarchy of classes of finite structures with increasingly more complex automorphism groups. Such a hierarchy can be defined in more than one way. For example, the $k$ th level of the hierarchy can consist of all structures having at least $k$ elements which are moved by some automorphism. Or we can consider, for any finite group $G$, all finite structures $\mathcal{M}$ such that $G$ is a subgroup of the group of automorphisms of $\mathcal{M}$; in this case the "hierarchy" is a partial order. In both cases, as well as variants of them, each "level" satisfies a logical limit law, but not a zero-one law (unless $k=0$ or $G$ is trivial). Moreover, the number of (labelled or unlabelled) $n$-element structures in one place of the hierarchy divided by the number of $n$-element structures in another place always converges to a rational number or to $\infty$ as $n \rightarrow \infty$. All instances of the respective result are proved by an essentially uniform argument.
\end{abstract}

2010 Mathematics Subject Classification $03 \mathrm{C} 13$ (primary); 60C05, 60F20 (secondary)

Keywords: finite model theory, limit law, zero-one law, random structure, automorphism group

\section{Introduction}

In a sequence of articles by Erdôs-Rényi [7], Fagin [9], Ford-Uhlenbeck [10], Harary [12] and Oberschelp [18] it has been shown that for any finite relational vocabulary (also called signature), the proportion of labelled (as well as unlabelled) $n$-element structures which are rigid, ie have no nontrivial automorphism, approaches 1 as (the positive integer) $n$ approaches infinity. By the work of Glebskii et. al. [11] and Fagin [8], for any sentence $\varphi$ the proportion of $n$-element structures (labelled or unlabelled) 
in which $\varphi$ is true approaches either 0 or 1 as $n$ tends to infinity. In other words, the class of finite structures satisfy a (labelled and unlabelled) zero-one law.

However, the asymptotic behaviour of nonrigid $n$-element structures appears to have been neglected, besides work of Cameron $[2,3]$ in the case of unlabelled undirected graphs. Possibly this is because the class of nonrigid finite structures make up to only a "measure zero" subclass of the class of all finite structures. Nevertheless, for any integer $k$ the number of (nonisomorphic) $n$-element structures with at least $k$ elements which are moved by some automorphism grows exponentially with $n$, and the same holds for the number of $n$-element structures whose automorphism group contains some specified group. (This follows from the proofs in Section 2.) But, more interestingly, consideration of finite structures whose automorphism group has a certain (minimum) complexity gives rise to an infinitude of natural classes of finite (nonrigid) structures with logical limit laws (Theorem 1.2). Each such class has the property that there are more than one but only finitely many "convergence points", all of which are rational; that is, there is a finite set $A$ of rational numbers such that $|A|>1$ and, for every sentence $\varphi$, the proportion of $n$-element structures in the class which satisfy $\varphi$ converges to a number in $A$. Moreover, in a sense that can be made precise, there are only finitely many (but more than one) "limit theories" of any such class, all of which are $\aleph_{0}$-categorical and simple with SU-rank one. ${ }^{1}$ It appears like the classes of nonrigid structures considered here are the first nontrivial and "naturally occurring" classes of finite structures with such limit law behaviour. ${ }^{2}$

Furthermore, for any two classes $\mathbf{C}$ and $\mathbf{K}$ of finite structures that are associated with some (minimum) complexity of the automorphism group, the number of (labelled or unlabelled) $n$-element structures which belong to $\mathbf{C}$ divided by the number of $n$-element structures which belong to $\mathbf{K}$ converges to a rational number or to $\infty$ as $n \rightarrow \infty$ (Theorem 1.1 and Remark 5.17).

In general, this study gives fairly complete answers, for any finite relational vocabulary with at least one relation symbol with arity at least 2 and for labelled as well as

\footnotetext{
${ }^{1}$ For any finite relational language with at least one symbol of arity at least 2 and integer $l \geq 2$, the class of all finite structures and the class of all (strongly) $l$-colourable finite structures (Kolaitis, Prömel and Rothschild [14] and Koponen [15]) have a zero-one law with a "limit/almost sure" theory which is $\aleph_{0}$-categorical and simple with SU-rank 1 . The class of all finite partial orders has a zero-one law (Compton [4]) with a limit theory which is probably $\aleph_{0}$-categorical (because the "height" of a finite partial order is almost always 3, see Kleitman and Rothschild [13]), although we have not checked this.

${ }^{2}$ A trivial example can constructed by adding a new unary relation symbol $R$ to a vocabulary with some relation symbol of arity at least 2 and letting the interpretation of $R$ be a singleton set in half of all $n$-element structures in the initial vocabulary and the empty set in the other half.
} 
unlabelled structures, to questions initiated by Cameron long ago (in particular Cameron [2, Theorems 1 and 2]), but also to other natural variations of his questions and to the problem of whether logical limit laws hold for classes of structures defined in terms of the complexity of their automorphism group.

A more detailed study, for any $m \in \mathbb{N}$, of the typical automorphism groups of finite structures such that at least $m$ elements are moved by some automorphism is carried out by the second author of this article in [16]. Roughly speaking, [16] shows that almost all finite structures with some minimum complexity of their automorphism group have as simple automorphism group as the minimum complexity allows.

Before stating the main results we introduce some basic terminology, notation and assumptions that will be used throughout. We fix a finite vocabulary, also called signature, $\left\{R_{1}, \ldots, R_{\rho}\right\}$ of (only) relation symbols where $R_{i}$ has arity $r_{i}$. Let $r=$ $\max \left\{r_{1}, \ldots, r_{\rho}\right\}$ and call $r$ the maximal arity. We always assume that $r \geq 2$, although this assumption is sometimes repeated. By a structure, we mean a structure for the above vocabulary, that is, a tuple $\mathcal{M}=\left(M, R_{1}^{\mathcal{M}}, \ldots, R_{\rho}^{\mathcal{M}}\right)$ where $M$ is a set, called the universe of $\mathcal{M}$, and, for each $i=1, \ldots, \rho, R_{i}^{\mathcal{M}} \subseteq M^{r_{i}}$. The relation $R_{i}^{\mathcal{M}}$ is called the interpretation of $R_{i}$ in $\mathcal{M}$. Many of the results depend only on the vocabulary, and in these cases they depend only on the sequence of arities $r_{1}, \ldots, r_{\rho}$. For every positive integer $n$ let $[n]=\{1, \ldots, n\}$, let $\mathbf{S}_{n}$ be the set of all structures with universe $[n]$, and let $\mathbf{S}=\bigcup_{n=1}^{\infty} \mathbf{S}_{n}$. For every structure $\mathcal{M}$, let $\operatorname{Aut}(\mathcal{M})$ denote the group of automorphisms of $\mathcal{M}$. (For basic model theory, see Marker [17] or Rothmaler [19].)

For groups $G$ and $H, G \cong H$ means that they are isomorphic (as abstract groups) and $G \leq H$ means that $G$ is isomorphic to a subgroup of $H$. For structures $\mathcal{M}$ and $\mathcal{N}$, $\mathcal{M} \cong \mathcal{N}$ means that they are isomorphic. Let $\mathbb{N}, \mathbb{N}^{+}, \mathbb{Q}$ and $\mathbb{R}$ denote the sets of nonnegative integers, positive integers, rational and real numbers, respectively.

Theorem 1.1 For any two finite groups $G$ and $H$, each one of the following limits exists in $\mathbb{Q} \cup\{\infty\}$ :

$$
\begin{aligned}
& \lim _{n \rightarrow \infty} \frac{\left|\left\{\mathcal{M} \in \mathbf{S}_{n}: H \leq \operatorname{Aut}(\mathcal{M})\right\}\right|}{\left|\left\{\mathcal{M} \in \mathbf{S}_{n}: G \leq \operatorname{Aut}(\mathcal{M})\right\}\right|}, \quad \lim _{n \rightarrow \infty} \frac{\left|\left\{\mathcal{M} \in \mathbf{S}_{n}: H \cong \operatorname{Aut}(\mathcal{M})\right\}\right|}{\left|\left\{\mathcal{M} \in \mathbf{S}_{n}: G \cong \operatorname{Aut}(\mathcal{M})\right\}\right|} \quad \text { and } \\
& \lim _{n \rightarrow \infty} \frac{\left|\left\{\mathcal{M} \in \mathbf{S}_{n}: G \cong \operatorname{Aut}(\mathcal{M})\right\}\right|}{\left|\left\{\mathcal{M} \in \mathbf{S}_{n}: G \leq \operatorname{Aut}(\mathcal{M})\right\}\right|}
\end{aligned}
$$

We introduce some more notation which will be used throughout the article. For a set $A,|A|$ denotes its cardinality and $\operatorname{Sym}(A)$ denotes the group of all permutations of $A$. If $f_{1}, \ldots, f_{k} \in \operatorname{Sym}(A)$ then $\left\langle f_{1}, \ldots, f_{k}\right\rangle$ denotes the subgroup of $\operatorname{Sym}(A)$ generated by 
$f_{1}, \ldots, f_{k}$,

$$
\operatorname{Spt}\left(f_{1}, \ldots, f_{k}\right)=\left\{a \in A: g(a) \neq a \text { for some } g \in\left\langle f_{1}, \ldots, f_{k}\right\rangle\right\}
$$

and $\operatorname{spt}\left(f_{1}, \ldots, f_{k}\right)=\left|\operatorname{Spt}\left(f_{1}, \ldots, f_{k}\right)\right|$. We call $\operatorname{Spt}\left(f_{1}, \ldots, f_{k}\right)$ the support of the sequence $f_{1}, \ldots, f_{k}$. For a finite structure $\mathcal{M}$ we let

$$
\begin{aligned}
\operatorname{spt}(\mathcal{M}) & =\max \{\operatorname{spt}(f): f \in \operatorname{Aut}(\mathcal{M})\}, \\
\operatorname{Spt}^{*}(\mathcal{M}) & =\{a \in M: a \in \operatorname{Spt}(f) \text { for some } f \in \operatorname{Aut}(\mathcal{M})\}, \text { and } \\
\operatorname{spt}^{*}(\mathcal{M}) & =\left|\operatorname{Spt}^{*}(\mathcal{M})\right| .
\end{aligned}
$$

The set $\operatorname{Spt}^{*}(\mathcal{M})$ is called the support of $\mathcal{M}$. Note that we always have $\operatorname{spt}(\mathcal{M}) \leq$ $\operatorname{spt}^{*}(\mathcal{M})$. Throughout, we use the following notation for $p, p^{\prime} \in \mathbb{N}$ :

$$
\begin{aligned}
\mathbf{S}_{n}(\mathrm{spt}=p) & =\left\{\mathcal{M} \in \mathbf{S}_{n}: \operatorname{spt}(\mathcal{M})=p\right\}, \\
\mathbf{S}_{n}(\mathrm{spt} \geq p) & =\left\{\mathcal{M} \in \mathbf{S}_{n}: \operatorname{spt}(\mathcal{M}) \geq p\right\}, \\
\mathbf{S}_{n}(\mathrm{spt} \leq p) & =\left\{\mathcal{M} \in \mathbf{S}_{n}: \operatorname{spt}(\mathcal{M}) \leq p\right\}, \\
\mathbf{S}_{n}\left(\operatorname{spt}^{*}=p\right) & =\left\{\mathcal{M} \in \mathbf{S}_{n}: \operatorname{spt}^{*}(\mathcal{M})=p\right\}, \\
\mathbf{S}_{n}\left(\mathrm{spt}^{*} \geq p\right) & =\left\{\mathcal{M} \in \mathbf{S}_{n}: \operatorname{spt}^{*}(\mathcal{M}) \geq p\right\}, \\
\mathbf{S}_{n}\left(\operatorname{spt}^{*} \leq p\right) & =\left\{\mathcal{M} \in \mathbf{S}_{n}: \operatorname{spt}^{*}(\mathcal{M}) \leq p\right\}, \\
\mathbf{S}_{n}\left(p \leq \operatorname{spt} \leq p^{\prime}\right) & =\left\{\mathcal{M} \in \mathbf{S}_{n}: p \leq \operatorname{spt}(\mathcal{M}) \leq p^{\prime}\right\} .
\end{aligned}
$$

Whenever $\mathbf{S}_{n}^{\prime} \subseteq \mathbf{S}_{n}$ is defined for $n \in \mathbb{N}^{+}$we let $\mathbf{S}^{\prime}=\bigcup_{n=1}^{\infty} \mathbf{S}_{n}^{\prime}$. The expression almost all $\mathcal{M} \in \mathbf{S}^{\prime}$ has property $P$ means that

$$
\lim _{n \rightarrow \infty} \frac{\mid\left\{\mathcal{M} \in \mathbf{S}_{n}^{\prime}: \mathcal{M} \text { has } P\right\} \mid}{\left|\mathbf{S}_{n}^{\prime}\right|}=1
$$

Suppose that $\mathbf{S}_{n}^{\prime} \subseteq \mathbf{S}_{n}$ for all $n \in \mathbb{N}^{+}$. We say that $\mathbf{S}^{\prime}=\bigcup_{n \in \mathbb{N}^{+}} \mathbf{S}_{n}^{\prime}$ has a limit law if for every first-order sentence $\varphi$ over the vocabulary, the proportion of $\mathcal{M} \in \mathbf{S}_{n}^{\prime}$ which satisfy $\varphi$ converges as $n \rightarrow \infty$. If the limit converges to 0 or 1 for every first-order sentence $\varphi$, then we say that $\mathbf{S}^{\prime}$ has a zero-one law.

Theorem 1.2 (i) For every finite group $G,\{\mathcal{M} \in \mathbf{S}: G \cong \operatorname{Aut}(\mathcal{M})\}$ and $\{\mathcal{M} \in \mathbf{S}: G \leq \operatorname{Aut}(\mathcal{M})\}$ have a limit law.

(ii) For every integer $m \geq 2, \mathbf{S}\left(\mathrm{spt}^{*}=m\right), \mathbf{S}(\mathrm{spt} \geq m)$ and $\mathbf{S}\left(\mathrm{spt}^{*} \geq m\right)$ have a limit law.

(iii) In each case of the previous parts there is a finite set $A \subseteq \mathbb{Q}$ such that, for every first-order sentence $\varphi$, the proportion of $n$-element structures of the kind considered which satisfy $\varphi$ converges to some $a \in A$ as $n \rightarrow \infty$. 
However, in each case of Theorem 1.2 we do not we have a zero-one law if $G$ is nontrivial, as explained in Remark 6.9.

Theorem 1.3 Theorems 1.1 and 1.2. also hold in the unlabelled case, that is if we only count structures up to isomorphism.

Remark 1.4 (Asymptotic estimates) The results, in particular Propositions 4.4 and 5.9 and Lemmas 4.2, 4.3, 5.3 and 5.8 give, in principle, a method of finding, for any finite group $G$, an asymptotic formula of the number of $\mathcal{M} \in \mathbf{S}_{n}$ such that $G \leq \operatorname{Aut}(\mathcal{M})$. The same is true if ' $\leq$ ' is replaced by ' $\cong$ ' or if we instead consider, for some arbitrary fixed integer $m \geq 2,\left|\mathbf{S}_{n}(\mathrm{spt} \geq m)\right|,\left|\mathbf{S}_{n}\left(\mathrm{spt}^{*} \geq m\right)\right|$ or $\left|\mathbf{S}_{n}\left(\mathrm{spt}^{*}=m\right)\right|$ as $n \rightarrow \infty$.

Remark 1.5 (Irreflexive and symmetric relations) (i) Suppose that every relation symbol is always interpreted as an irreflexive relation, that is, if $\mathcal{M} \models R_{i}\left(a_{1}, \ldots, a_{r_{i}}\right)$ then $a_{j} \neq a_{j^{\prime}}$ whenever $j \neq j^{\prime}$. Then Theorems $1.1-1.3$ remain true, but some modifications have to be made in some proofs and in some technical results of the article.

(ii) Suppose that every relation symbol is always interpreted as an irreflexive and symmetric relation, where the later means that if $\mathcal{M} \models R_{i}\left(a_{1}, \ldots, a_{r_{i}}\right)$ then $\mathcal{M} \models$ $R_{i}\left(a_{\pi(1)}, \ldots, a_{\pi\left(r_{i}\right)}\right)$ for every permutation $\pi$ of $\left[r_{i}\right]$. Again Theorems $1.1-1.3$ remain true, with minor modifications in some proofs and technical results.

Here follows an outline of the article. We deal with labelled structures until the last section, where we show why the main results also hold for unlabelled structures. In Section 2 we show that for every $m \in \mathbb{N}$ there is a number $t$, depending only on $m$ and the vocabulary, such that almost all $\mathcal{M} \in \mathbf{S}(\mathrm{spt} \geq m)$ have no automorphism the support of which contains more than $t$ elements. In Section 3 we show, by a Ramsey type argument, that if $\mathcal{M}$ is finite and for every $f \in \operatorname{Aut}(\mathcal{M}), \operatorname{spt}(f) \leq t$, then there are at most $t^{t+2}$ elements $a \in M$ such that $g(a) \neq a$ for some $g \in \operatorname{Aut}(\mathcal{M})$. More briefly, with the notation after Theorem 1.1: if $\operatorname{spt}(\mathcal{M}) \leq t$ then $\operatorname{spt}^{*}(\mathcal{M}) \leq t^{t+2}$. A consequence of these results is that for every $m \in \mathbb{N}$ there is $T \in \mathbb{N}$ such that almost all $\mathcal{M} \in \mathbf{S}(\mathrm{spt} \geq m)$ have the property that at most $T$ elements are moved by some automorphism. (In the case of unlabelled undirected graphs this was proved, in a different way by Cameron [2].)

Section 4 considers asymptotic estimates that are needed later. In this section, a structure $\mathcal{A} \in \mathbf{S}$ and subgroup $H$ of $\operatorname{Aut}(\mathcal{A})$ are given and an asymptotic estimate is proved for the number of $\mathcal{M} \in \mathbf{S}_{n}$ such that $\mathcal{M}\left\lceil\operatorname{spt}^{*}(\mathcal{M}) \cong \mathcal{A}\right.$ and there is an isomorphism $f: \mathcal{A} \rightarrow \mathcal{M}\left\lceil\operatorname{spt}^{*}(\mathcal{M})\right.$ such that $H_{f}=\left\{f \sigma f^{-1}: \sigma \in H\right\}$ is a subgroup of the group 
$\left\{g\left\lceil\operatorname{Spt}^{*}(\mathcal{M}): g \in \operatorname{Aut}(\mathcal{M})\right\}\right.$, which implies that $\operatorname{Aut}(\mathcal{M})$ contains a copy of $H$. The set of such structures is denoted $\mathbf{S}_{n}(\mathcal{A}, H)$. Sets of this sort are the "building blocks" of other sets of structures considered here, in the sense that almost all structures of any set of structures in the main theorems belong to a finite union of sets of the form $\bigcup_{n=1}^{\infty} \mathbf{S}_{n}(\mathcal{A}, H)$. In Section 5 we use the results from previous sections, in particular the asymptotic estimate of $\mathbf{S}_{n}(\mathcal{A}, H)$, to prove Theorem 1.1, in the form of Propositions 5.10, 5.15 and 5.16 .

Theorem 1.2, about logical limit laws, is proved in Section 6. Again, the set $\mathbf{S}_{n}(\mathcal{A}, H)$ plays a central role. In fact, the main task is to prove that $\mathbf{S}(\mathcal{A}, H)$ has a zero-one law. This and Proposition 5.9 implies Theorem 1.2. The final Section 7 shows why all main results also hold for unlabelled structures. This is summarised in Theorem 7.7 which implies Theorem 1.3.

Terminology and notation 1.6 We use the calligraphic letters $\mathcal{A}, \mathcal{B}, \mathcal{C}, \mathcal{M}, \mathcal{N}$ to denote structures and the corresponding noncalligraphic letters $A, B, C, M, N$ to denote their universes. Usually the universe will be $[n]=\{1, \ldots, n\}$ for some $n \in \mathbb{N}^{+}$. We sometimes write $\bar{a}$ to denote a finite tuple $\left(a_{1}, \ldots, a_{n}\right)$, and if $\bar{a}=\left(a_{1}, \ldots, a_{n}\right)$ and $\bar{b}=\left(b_{1}, \ldots, b_{m}\right)$, then we let $\bar{a} \bar{b}=\left(a_{1}, \ldots, a_{n}, b_{1}, \ldots, b_{m}\right)$. If $\mathcal{M}$ is a structure and $A \subseteq M$, then $\mathcal{M} \uparrow A$ denotes the substructure of $\mathcal{M}$ with universe $A$.

Let $H$ and $H^{\prime}$ be permutation groups on sets $\Omega$ and $\Omega^{\prime}$, respectively. A bijection $f: \Omega \rightarrow \Omega^{\prime}$ is called an isomorphism from $H$ to $H^{\prime}$ as permutation groups if $H^{\prime}=\left\{f h f^{-1}: h \in H\right\}$. We say that $H$ and $H^{\prime}$ are isomorphic as permutation groups if such $f$ exists; this clearly implies that they are isomorphic as abstract groups. We let $H \cong_{P} H^{\prime}$ mean that $H$ and $H^{\prime}$ are isomorphic as permutation groups. If $f: A \rightarrow B$ is a function and $X \subseteq A$, then $f\lceil X$ denotes the restriction of $f$ to $X$. If $H$ is a permutation group on $\Omega$ and $X \subseteq \Omega$ is the union of some of the orbits of $H$ on $\Omega$, then we define $H\lceil X=\{h\lceil X: h \in H\}$, which is a permutation group on $X$, and we call $H\lceil X$ the restriction of $H$ to $X$.

If $f$ is a permutation of $\Omega$ then $a \in \Omega$ is called a fixed point of $f$ if $f(a)=a$. If $H$ is a group of permutations of $\Omega$ then $a \in \Omega$ is called a fixed point of $H$ if $a$ is a fixed point of every $h \in H$. For a structure $\mathcal{A}, a \in A$ is called a fixed point of $\mathcal{A}$ if $a$ is a fixed point of $\operatorname{Aut}(\mathcal{A})$. For any nonempty set $\Omega, \operatorname{Sym}(\Omega)$ denotes the symmetric group of $\Omega$, ie the group of all permutations of $\Omega$, and $\operatorname{Sym}_{n}=\operatorname{Sym}([n])$.

If $G$ is a group and $g_{1}, \ldots, g_{n} \in G$ then $\left\langle g_{1}, \ldots, g_{n}\right\rangle$ denotes the subgroup of $G$ generated by $g_{1}, \ldots, g_{n}$. For a permutation group $G$ on a set $\Omega$, if $x \in \Omega$ then the orbit of $x$ (with respect to $G$ ) is the set $\{g(x): g \in G\}$ and any set 
of this form is called an orbit of $G$. Let $\operatorname{Orb}(G)$ be the set of orbits of $G$ and $\operatorname{orb}(G)=|\operatorname{Orb}(G)|$. Such $G$ also acts on $\Omega^{m}$, the set of ordered $m$-tuples of elements from $\Omega$, by the action $g\left(a_{1}, \ldots, a_{m}\right)=\left(g\left(a_{1}\right), \ldots, g\left(a_{m}\right)\right)$ for every $g \in G$ and $\left(a_{1}, \ldots, a_{m}\right) \in \Omega^{m}$. When referring to "the orbits of $G$ on $\Omega^{m}$ " we mean the orbits with respect to this action, unless something else is said. We let $\operatorname{Orb}^{m}(G)$ be the set of orbits of $G$ on $\Omega^{m}$ and $\operatorname{orb}^{m}(G)=\left|\operatorname{Orb}^{m}(G)\right|$. For $\pi_{1}, \ldots, \pi_{k} \in \operatorname{Sym}_{n}$ we let $\operatorname{Orb}\left(\pi_{1}, \ldots, \pi_{k}\right)=\operatorname{Orb}\left(\left\langle\pi_{1}, \ldots, \pi_{k}\right\rangle\right), \operatorname{orb}\left(\pi_{1}, \ldots, \pi_{k}\right)=\operatorname{orb}\left(\left\langle\pi_{1}, \ldots, \pi_{k}\right\rangle\right)$, $\operatorname{Orb}^{m}\left(\pi_{1}, \ldots, \pi_{k}\right)=\operatorname{Orb}^{m}\left(\left\langle\pi_{1}, \ldots, \pi_{k}\right\rangle\right)$ and

$\operatorname{orb}^{m}\left(\pi_{1}, \ldots, \pi_{k}\right)=\operatorname{orb}^{m}\left(\left\langle\pi_{1}, \ldots, \pi_{k}\right\rangle\right)$. For unexplained notions such as 'action, orbit' etc., see for example Dixon and Mortimer [5].

We will also use the terminology and notation that was introduced between Theorems 1.1 -1.3 as well as the following notation: if $f_{1}, \ldots, f_{k}$ are permutations of $[n]$, then

$$
\mathbf{S}_{n}\left(f_{1}, \ldots, f_{k}\right)=\left\{\mathcal{M} \in \mathbf{S}_{n}: f_{1}, \ldots, f_{k} \in \operatorname{Aut}(\mathcal{M})\right\} .
$$

By $f(n) \sim g(n)($ as $n \rightarrow \infty)$ we mean that $f(n) / g(n) \rightarrow 1$ as $n \rightarrow \infty$. The parameter $n$ will (with some exceptions in Section 3) always be the number of elements in structures we consider and any $o(\ldots)$ or $\mathcal{O}(\ldots)$ will be with respect to $n$ as it approaches infinity. It will be convenient to use the notation $\exp _{2}(x)=2^{x}$.

\section{Upper bounds of the support of automorphisms}

The main result of this section, Proposition 2.3, is that for any $m \in \mathbb{N}$ there is $t \in \mathbb{N}$ such that the proportion of $\mathcal{M} \in \mathbf{S}_{n}(\operatorname{spt} \geq m)$ such that $\operatorname{spt}(\mathcal{M}) \leq t$ approaches 1 as $n \rightarrow \infty$. We also derive a couple of corollaries of this which are important for the rest of the article. The following elementary result, often called Burnside's Lemma or Theorem $^{3}$, will be used. Proofs are found in Burnside [1] and Dixon and Mortimer [5], for example.

Proposition 2.1 If $G$ is a group of permutations of a finite set $M$ then

$$
\operatorname{orb}(G)=\frac{1}{|G|} \sum_{g \in G}|\{a \in M: g(a)=a\}|
$$

Recall that $[n]=\{1, \ldots, n\}$ and by $[n]^{r}$ we denote the set of ordered $r$-tuples of elements from $[n]$.

\footnotetext{
${ }^{3}$ But was actually proved earlier by Cauchy and Frobenius, according to Dixon and Mortimer [5]
} 
Lemma 2.2 Suppose that $d, n \in \mathbb{N}^{+}, \pi_{1}, \ldots, \pi_{s} \in \operatorname{Sym}_{n}$ and $\operatorname{spt}\left(\pi_{1}, \ldots, \pi_{s}\right)=p$. Then

$$
\frac{n^{d}+(p !-1)(n-p)^{d}}{p !} \leq \operatorname{orb}^{d}\left(\pi_{1}, \ldots, \pi_{s}\right) \leq n^{d}-\frac{p n^{d-1}}{2} .
$$

Proof For each $\pi \in \operatorname{Sym}_{n}$ let $\tilde{\pi} \in \operatorname{Sym}\left([n]^{d}\right)$ be defined by $\tilde{\pi}\left(x_{1}, \ldots, x_{d}\right)=$ $\left(\pi\left(x_{1}\right), \ldots, \pi\left(x_{d}\right)\right)$. We consider the subgroup $G=\left\langle\pi_{1}, \ldots, \pi_{s}\right\rangle$ of $S_{y m}$ and the subgroup $\widetilde{G}=\left\langle\widetilde{\pi}_{1}, \ldots, \widetilde{\pi}_{s}\right\rangle$ of $\operatorname{Sym}\left([n]^{d}\right)$. The map $\pi \mapsto \widetilde{\pi}$ is an isomorphism from $G$ onto $\widetilde{G}$, so $\operatorname{spt}(\widetilde{G})=p$, and hence $|\widetilde{G}| \leq p$ !. Note that $\operatorname{orb}(\widetilde{G})=\operatorname{orb}^{d}(G)=$ $\operatorname{orb}^{d}\left(\pi_{1}, \ldots, \pi_{s}\right)$. Also observe that, by the assumption that $\operatorname{spt}(G)=\operatorname{spt}\left(\pi_{1}, \ldots, \pi_{s}\right)=$ $p$, every $g \in G$ has at least $n-p$ fixed points. Therefore every $g \in \widetilde{G}$ has at least $(n-p)^{d}$ fixed points. In particular, the identity permutation has $n^{d}$ fixed points. Therefore we get, by also using Proposition 2.1,

$$
\begin{aligned}
\operatorname{orb}^{d}\left(\pi_{1}, \ldots, \pi_{s}\right) & =\operatorname{orb}(\widetilde{G})=\frac{1}{|\widetilde{G}|} \sum_{\widetilde{g} \in \widetilde{G}}\left|\left\{\bar{a} \in[n]^{d}: \widetilde{g}(\bar{a})=\bar{a}\right\}\right| \\
& \geq \frac{(|\widetilde{G}|-1)(n-p)^{d}+n^{d}}{|\widetilde{G}|}=(n-p)^{d}+\frac{n^{d}-(n-p)^{d}}{|\widetilde{G}|} \\
& \geq(n-p)^{d}+\frac{n^{d}-(n-p)^{d}}{p !}=\frac{n^{d}+(p !-1)(n-p)^{d}}{p !}
\end{aligned}
$$

On the other hand we also have that

$$
\operatorname{orb}(G) \leq(n-p)+\frac{p}{2}=n-\frac{p}{2}
$$

which implies that

$$
\operatorname{orb}^{d}\left(\pi_{1}, \ldots, \pi_{s}\right)=\operatorname{orb}(\widetilde{G}) \leq \operatorname{orb}(G) \cdot n^{d-1} \leq n^{d}-\frac{p n^{d-1}}{2},
$$

because if $\left(a_{1}, \ldots, a_{d}\right)$ and $\left(b_{1}, \ldots, b_{d}\right)$ belong to the same orbit of $\widetilde{G}$ then $a_{1}$ and $b_{1}$ belong to the same orbit of $G$.

Recall that $r \geq 2$ is the maximal arity among relation symbols in the vocabulary.

Proposition 2.3 Suppose that $m, t \in \mathbb{N}, f_{1}, \ldots, f_{s} \in \operatorname{Sym}_{n}$ and $\operatorname{spt}\left(f_{1}, \ldots, f_{s}\right)=m$. For all sufficiently large $n$ the following holds, where $k$ is the number of $r$-ary relation symbols and the bound $\mathcal{O}()$ depends only on $m, t$ and the vocabulary:

$$
\frac{\left|\mathbf{S}_{n}(\mathrm{spt} \geq t)\right|}{\left|\mathbf{S}_{n}\left(f_{1}, \ldots, f_{s}\right)\right|} \leq \exp _{2}\left(k \frac{(2(m !-1) r m-(t-1) m !) n^{r-1}}{2(m !)} \pm \mathcal{O}\left(n^{r-2}\right)\right) .
$$

Hence, if $t>2 r(m !-1) m / m !+1$ then the quotient approaches 0 as $n \rightarrow \infty$. 
Proof For each $i=1, \ldots, r$, let $k_{i}$ be the number of $i$-ary relation symbols. Suppose that $m, t \in \mathbb{N}, f_{1}, \ldots, f_{s} \in \operatorname{Sym}_{n}$ and $\operatorname{spt}\left(f_{1}, \ldots, f_{s}\right)=m$. Observe that for every $i$ and every $i$-ary relation symbol $R$ we have: if $\bar{a}, \bar{b} \in[n]^{i}$ belong to the same orbit of $\left\langle f_{1}, \ldots, f_{s}\right\rangle$ and $\mathcal{M} \in \mathbf{S}_{n}\left(f_{1}, \ldots, f_{s}\right)$, then $\mathcal{M} \models R(\bar{a})$ if and only if $\mathcal{M} \models R(\bar{b})$. Since this is the only restriction on members of $\mathbf{S}_{n}\left(f_{1}, \ldots, f_{s}\right)$ we get

$$
\left|\mathbf{S}_{n}\left(f_{1}, \ldots, f_{s}\right)\right|=\exp _{2}\left(\sum_{i=1}^{r} k_{i} \operatorname{orb}^{i}\left(f_{1}, \ldots, f_{s}\right)\right) .
$$

For every $\mathcal{M} \in \mathbf{S}_{n}(\operatorname{spt} \geq t)$ there exists $\pi \in \operatorname{Aut}(\mathcal{M})$ such that $\operatorname{Spt}(\pi) \geq t$ and therefore

$$
\left|\mathbf{S}_{n}(\mathrm{spt} \geq t)\right| \leq \sum_{\substack{\pi \in S y m_{n} \\ \operatorname{spt}(\pi) \geq t}}\left|\mathbf{S}_{n}(\pi)\right|=\sum_{\substack{\pi \in S y m_{n} \\ \operatorname{spt}(\pi) \geq t}} \exp _{2}\left(\sum_{i=1}^{r} k_{i} \operatorname{orb}^{i}(\pi)\right) .
$$

By first applying Lemma 2.2 on $f_{1}, \ldots, f_{s}$ and then on an arbitrary $\pi \in S y m_{n}$ we get, for each $i=1, \ldots, r$,

$$
\begin{gathered}
\frac{n^{i}+(m !-1)(n-m)^{i}}{m !} \leq \operatorname{orb}^{i}\left(f_{1}, \ldots, f_{s}\right), \quad \text { and } \\
\operatorname{orb}^{i}(\pi) \leq n^{i}-\frac{n^{i-1} \operatorname{spt}(\pi)}{2} \quad \text { for every } \pi \in \text { Sym }_{n} .
\end{gathered}
$$

A straightforward computation ${ }^{4}$ shows that for all sufficiently large $n$

$$
\begin{aligned}
& \sum_{j=t}^{n} \exp _{2}\left(j \log _{2} n-j \sum_{i=1}^{r} k_{i} \frac{n^{i-1}}{2}\right) \\
\leq & \exp _{2}\left(-k_{r} \frac{(t-1) n^{r-1}}{2} \pm \mathcal{O}\left(n^{r-2}+\log _{2} n\right)\right),
\end{aligned}
$$

where the bound $\mathcal{O}()$ depends only on the vocabulary. Notice that the number of $\pi \in \operatorname{Sym}_{n}$ with $\operatorname{spt}(\pi)=j$ is $\left(\begin{array}{l}n \\ j\end{array}\right) j ! \leq n^{j}$. By also using (2-1)-(2-5) we now get

\footnotetext{
${ }^{4}$ Set $a=\exp _{2}\left(\log _{2} n-\sum_{i=1}^{r} k_{i} \frac{n^{i-1}}{2}\right)$ and we have $\sum_{j=t}^{n} a^{j} \leq a^{t} /(1-a) \leq a^{t-1}$ if $n$ is large enough.
} 


$$
\begin{aligned}
& \frac{\left|\mathbf{S}_{n}(\operatorname{spt} \geq t)\right|}{\left|\mathbf{S}_{n}\left(f_{1}, \ldots, f_{s}\right)\right|} \leq \sum_{\substack{\pi \in S_{\operatorname{Sym}} \\
\operatorname{spt}(\pi) \geq t}} \exp _{2}\left(\sum_{i=1}^{r} k_{i} \operatorname{orb}^{i}(\pi)-\sum_{i=1}^{r} k_{i} \operatorname{orb}^{i}\left(f_{1}, \ldots, f_{s}\right)\right) \\
& \leq \sum_{\substack{\pi \in S y m_{n} \\
\operatorname{spt}(\pi) \geq t}} \exp _{2}\left(\sum_{i=1}^{r} k_{i}\left[n^{i}-\frac{n^{i-1} \operatorname{spt}(\pi)}{2}\right]-\sum_{i=1}^{r} k_{i} \frac{n^{i}+(m !-1)(n-m)^{i}}{m !}\right) \\
& \leq \sum_{j=t}^{n} n^{j} \exp _{2}\left(\sum_{i=1}^{r} k_{i}\left[n^{i}-\frac{j n^{i-1}}{2}\right]-\sum_{i=1}^{r} k_{i} \frac{n^{i}+(m !-1)(n-m)^{i}}{m !}\right) \\
& =\exp _{2}\left(\sum_{i=1}^{r} k_{i}\left[n^{i}-\frac{n^{i}+(m !-1)(n-m)^{i}}{m !}\right]\right) \sum_{j=t}^{n} \exp _{2}\left(j \log _{2} n-j \sum_{i=1}^{r} k_{i} \frac{n^{i-1}}{2}\right) \\
& \leq \exp _{2}\left(k_{1} \frac{(m !-1) m}{m !}+\sum_{i=2}^{r} k_{i} \frac{(m !-1) i m n^{i-1} \pm \mathcal{O}\left(n^{i-2}\right)}{m !}\right) . \\
& \quad \exp _{2}\left(-k_{r} \frac{(t-1) n^{r-1}}{2} \pm \mathcal{O}\left(n^{r-2}+\log _{2} n\right)\right) \\
& =\exp _{2}\left(k_{r} \frac{(2(m !-1) r m-(t-1) m !) n^{r-1}}{2(m !)} \pm \mathcal{O}\left(n^{r-2}+\log _{2} n\right)\right) .
\end{aligned}
$$

Remark 2.4 Suppose that we require that a relation symbol $R_{i}$ of arity $r_{i} \geq 2$ is always interpreted as an irreflexive and symmetric relation. Then we need to use a modification of Lemma 2.2 where, for $\pi_{1}, \ldots, \pi_{s} \in S y m_{n}$, we consider the orbits of $G=\left\langle\pi_{1}, \ldots, \pi_{s}\right\rangle$ on the set of $r_{i}$-subsets of $[n]$ by the action $g\left(\left\{a_{1}, \ldots, a_{r_{i}}\right\}\right)=\left\{g\left(a_{1}\right), \ldots, g\left(a_{s}\right)\right\}$ for every $g \in G$ and $r_{i}$-subset $\left\{a_{1}, \ldots, a_{r_{i}}\right\} \subseteq[n]$. By slightly modifying the proof of Lemma 2.2 one gets that if $q$ is the number of orbits of $G$ by its action on the set of $r_{i}$-subsets of $[n]$, then

$$
\frac{\left(\begin{array}{l}
n \\
d
\end{array}\right)-(p !-1)\left(\begin{array}{c}
n-p \\
d
\end{array}\right)}{p !} \leq q \leq n\left(\begin{array}{l}
n \\
d
\end{array}\right)-\frac{p}{2}\left(\begin{array}{c}
n \\
d-1
\end{array}\right) .
$$

By using this when estimating (the appropriate analogues of $\operatorname{orb}^{i}(\pi)$ and $\operatorname{orb}^{i}\left(f_{1}, \ldots, f_{s}\right)$ in the proof of Proposition 2.3 for each $i$-ary relation symbol (where $i \geq 2$ ) that is always interpreted as an irreflexive and symmetric relation, one gets a similar upper bound, by a bit more involved computations. Similar adaptations work if we require that some relation symbols are always interpreted as irreflexive, but not necessarily symmetric, relations. 
Corollary 2.5 Let $m \in \mathbb{N}$. If $t>2 r(m !-1) m / m !+1$ then

$$
\lim _{n \rightarrow \infty} \frac{\left|\mathbf{S}_{n}(\mathrm{spt} \geq t)\right|}{\left|\mathbf{S}_{n}(\mathrm{spt} \geq m)\right|}=\lim _{n \rightarrow \infty} \frac{\left|\mathbf{S}_{n}(\mathrm{spt} \geq t)\right|}{\left|\mathbf{S}_{n}\left(\mathrm{spt}^{*} \geq m\right)\right|}=0 .
$$

Proof This follows immediately from Proposition 2.3, because if $f \in S y m_{n}$ and $\operatorname{spt}(f)=m$, then $\mathbf{S}_{n}(f) \subseteq \mathbf{S}_{n}(\mathrm{spt} \geq m) \subseteq \mathbf{S}_{n}\left(\mathrm{spt}^{*} \geq m\right)$.

Corollary 2.6 Suppose that $G$ is a finite group which is isomorphic to a group of permutations of $[m]$. If $t=2 r(m !-1) m / m !+1$ then

$$
\lim _{n \rightarrow \infty} \frac{\left.\mid\left\{\mathcal{M} \in \mathbf{S}_{n}: G \leq \operatorname{Aut}(\mathcal{M}) \text { and } \operatorname{spt}(\mathcal{M}) \leq t\right)\right\} \mid}{\left|\left\{\mathcal{M} \in \mathbf{S}_{n}: G \leq \operatorname{Aut}(\mathcal{M})\right\}\right|}=1
$$

Proof Let $H=\left\{h_{1}, \ldots, h_{s}\right\}$ be a permutation group on [m] such that $H \cong G$. Let $t=2 r(m !-1) m / m !+1$. Extend each $h_{i}$ to a permutation $h_{i}^{\prime}$ of $[n]$ by letting $h_{i}^{\prime}(j)=j$ for every $j>m$ and $h_{i}^{\prime}(j)=h_{i}(j)$ for every $j \leq m$. Observe that for every $\mathcal{M} \in \mathbf{S}_{n}\left(h_{1}^{\prime}, \ldots, h_{s}^{\prime}\right), G \leq \operatorname{Aut}(\mathcal{M})$. From Proposition 2.3 we get

$$
\frac{\mid\left\{\mathcal{M} \in \mathbf{S}_{n}: G \leq \operatorname{Aut}(\mathcal{M}) \text { and } \operatorname{spt}(\mathcal{M})>t\right\} \mid}{\left|\left\{\mathcal{M} \in \mathbf{S}_{n}: G \leq \operatorname{Aut}(\mathcal{M})\right\}\right|} \leq \frac{\left|\mathbf{S}_{n}(\mathrm{spt}>t)\right|}{\left|\mathbf{S}_{n}\left(h_{1}^{\prime}, \ldots, h_{s}^{\prime}\right)\right|} \rightarrow 0,
$$

as $n \rightarrow \infty$.

\section{Upper bounds of the support of structures}

In this section we prove that for every $t \in \mathbb{N}$ there is $T \in \mathbb{N}$, depending only on $t$, such that for every finite structure $\mathcal{M}$, if $\operatorname{spt}(\mathcal{M}) \leq t$ then $\operatorname{spt}^{*}(\mathcal{M}) \leq T$. In other words, if no automorphism of $\mathcal{M}$ moves more than $t$ elements, then not more than $T$ elements of $\mathcal{M}$ are moved by some automorphism. This is stated by Proposition 3.5. Corollaries 3.7 and 3.8 will be used in later sections.

Definition 3.1 Let $\mathcal{M} \in \mathbf{S}$ and $X \subseteq M$.

(i) For $f \in \operatorname{Aut}(\mathcal{M})$ let $d(f, X)=|\operatorname{Spt}(f)-X|$.

(ii) We call $f \in \operatorname{Aut}(\mathcal{M})$ maximal if for all $g \in \operatorname{Aut}(\mathcal{M})$, if $\operatorname{Spt}(f) \subseteq \operatorname{Spt}(g)$ then $\operatorname{Spt}(f)=\operatorname{Spt}(g)$.

(iii) Let $\operatorname{Aut}^{*}(\mathcal{M})=\{f \in \operatorname{Aut}(\mathcal{M}): f$ is maximal $\}$.

(iv) For $\mathcal{M} \in \mathbf{S}$, a sequence $f_{0}, \ldots, f_{n} \subseteq \operatorname{Aut}^{*}(\mathcal{M})$ is called a special sequence of automorphisms of $\mathcal{M}$ if it satisfies the following condition: 
For each $k=0, \ldots, n-1$,

$$
d\left(f_{k+1}, \operatorname{Spt}\left(f_{0}, \ldots, f_{k}\right)\right)=\max _{g \in \operatorname{Aut}^{*}(\mathcal{M})} d\left(g, \operatorname{Spt}\left(f_{0}, \ldots, f_{k}\right)\right) .
$$

Notation 3.2 Whenever a special sequence of automorphisms $f_{0}, \ldots, f_{n} \in \operatorname{Aut}^{*}(\mathcal{M})$, $k \leq n$ and $g \in \operatorname{Aut}(\mathcal{M})$ are given, then we may use the abbreviation

$$
d_{k}(g)=d\left(g, \operatorname{Spt}\left(f_{0}, \ldots, f_{k}\right)\right) .
$$

The following lemma states some basic facts about special sequences of automorphisms.

Lemma 3.3 Let $\mathcal{M} \in \mathbf{S}$ and let $f_{0}, \ldots, f_{n} \in \operatorname{Aut}^{*}(\mathcal{M})$ be a special sequence of automorphisms. Then

(1) for all $0 \leq k \leq n$ and all $g \in \operatorname{Aut}(\mathcal{M}), d_{k}(g) \geq d_{k+1}(g)$,

(2) if $k+1 \leq p \leq n$ then $d_{k}\left(f_{k+1}\right) \geq d_{k}\left(f_{p}\right)$ and

(3) if $0 \leq k<n$ and $d_{k}\left(f_{k+1}\right)=0$ then for all $g \in \operatorname{Aut}^{*}(\mathcal{M}), \operatorname{Spt}(g) \subseteq$ $\operatorname{Spt}\left(f_{1}, \ldots, f_{k}\right)$.

Proof Let $\mathcal{M} \in \mathbf{S}$ and let $f_{0}, \ldots, f_{n} \in \operatorname{Aut}^{*}(\mathcal{M})$ be a special sequence of automorphisms.

(1) Suppose that $g \in \operatorname{Aut}(\mathcal{M})$. As $\operatorname{Spt}\left(f_{0}, \ldots, f_{k}\right) \subseteq \operatorname{Spt}\left(f_{0}, \ldots, f_{k+1}\right)$ we get

$$
\left|\operatorname{Spt}(g) \backslash \operatorname{Spt}\left(f_{0}, \ldots, f_{k}\right)\right| \geq\left|\operatorname{Spt}(g) \backslash \operatorname{Spt}\left(f_{0}, \ldots, f_{k+1}\right)\right|,
$$

that is, $d_{k}(g) \geq d_{k+1}(g)$.

(2) Suppose that $k+1 \leq p \leq n$. Since

$$
d_{k}\left(f_{k+1}\right)=\max _{g \in \operatorname{Aut}^{*}(\mathcal{M})} d_{k}(g)
$$

we get $d_{k}\left(f_{k+1}\right) \geq d_{k}\left(f_{p}\right)$.

(3) If $0 \leq k<n$ and $d_{k}\left(f_{k+1}\right)=0$, then $\max _{g \in \operatorname{Aut}^{*}(\mathcal{M})} d_{k}(g)=0$, so $\operatorname{Spt}(g) \subseteq$ $\operatorname{Spt}\left(f_{1}, \ldots, f_{k}\right)$ for every $g \in \operatorname{Aut}^{*}(\mathcal{M})$.

Now to a less obvious claim:

Lemma 3.4 Let $\mathcal{M} \in \mathbf{S}$. Suppose that $f_{0}, \ldots, f_{n} \in \operatorname{Aut}^{*}(\mathcal{M})$ is a special sequence and $1 \leq k<p \leq n$. If $d_{k}\left(f_{p}\right)>0$ then there is $x \in \operatorname{Spt}\left(f_{k}\right) \backslash \operatorname{Spt}\left(f_{0}, \ldots, f_{k-1}\right)$ such that $x \notin \operatorname{Spt}\left(f_{p}\right)$. 
Proof Let $\mathcal{M} \in \mathbf{S}$, let $f_{0}, \ldots, f_{n} \in \operatorname{Aut}^{*}(\mathcal{M})$ be a special sequence and suppose that $1 \leq k<p$ and $d_{k}\left(f_{p}\right)>0$. We use the abbreviations $\operatorname{Spt}(k)=\operatorname{Spt}\left(f_{k}\right)$ and $\operatorname{Spt}(0, \ldots, k)=\operatorname{Spt}\left(f_{0}, \ldots, f_{k}\right)$. Let

$$
X=\operatorname{Spt}(k) \backslash \operatorname{Spt}(0, \ldots, k-1) .
$$

For a contradiction, we assume that $X \subseteq \operatorname{Spt}(p)$. Since $d_{k}\left(f_{p}\right)>0$ we know that there is an element $a \in \operatorname{Spt}(p)$ such that $a \notin \operatorname{Spt}(0, \ldots, k)$. Then Lemma 3.3 (1) together with $d_{k}\left(f_{p}\right)>0$ gives us that $d_{k-1}\left(f_{p}\right)>0$. By Lemma 3.3 (2) we get $d_{k-1}\left(f_{k}\right)>0$, which implies that $X \neq \emptyset$. Also notice that $a \notin X$, by the choice of $a$. From the definition of $X$ and the assumption that $X \subseteq \operatorname{Spt}(p)$ it follows that $X \subseteq \operatorname{Spt}(p) \backslash \operatorname{Spt}(0, \ldots, k-1)$. By the choice of $a$ we have $a \in \operatorname{Spt}(p) \backslash \operatorname{Spt}(0, \ldots, k-1)$, so we get

$$
X \cup\{a\} \subseteq \operatorname{Spt}(p) \backslash \operatorname{Spt}(0, \ldots, k-1),
$$

and recall that $a \notin X$. Hence we get

$$
\begin{aligned}
d_{k-1}(k) & =|\operatorname{Spt}(k)-\operatorname{Spt}(0, \ldots, k-1)|=|X| \\
& <|X \cup\{a\}| \leq|\operatorname{Spt}(p) \backslash \operatorname{Spt}(0, \ldots, k-1)|=d_{k-1}(p),
\end{aligned}
$$

ie $d_{k-1}(k)<d_{k-1}(p)$ which contradicts Lemma $3.3(2)$.

The next proposition tells that, for each $k \geq 2, \mathbf{S}(\operatorname{spt} \leq k) \subseteq \mathbf{S}\left(\mathrm{spt}^{*} \leq k^{k+2}\right)$.

Proposition 3.5 For every integer $k \geq 2$ and every $\mathcal{M} \in \mathbf{S}($ spt $\leq k)$ we have $\operatorname{spt}^{*}(\mathcal{M}) \leq k^{k+2}$.

Proof Fix any integer $k \geq 2$. For $i=0, \ldots, k$, let $l_{i}=k^{k-i+1}$. Note that $l_{0}=k^{k+1}$ and $l_{i}=k l_{i+1}$ for each $i$. Suppose that $\mathcal{M} \in \mathbf{S}(\mathrm{spt} \leq k)$ and, for a contradiction, that $\operatorname{spt}^{*}(\mathcal{M})>k^{k+2}$.

By definition, any $f_{0} \in \operatorname{Aut}^{*}(\mathcal{M})$ is a special sequence of length 1 . Now let $f_{0}, \ldots, f_{n} \in$ $\operatorname{Aut}^{*}(\mathcal{M})$ be any special sequence and suppose that $n<l_{0}$. By the assumption that $\mathcal{M} \in \mathbf{S}($ spt $\leq k)$ we have $\left|\operatorname{Spt}\left(f_{0}, \ldots, f_{n}\right)\right| \leq k l_{0}=k^{k+2}$. From the assumption that $\operatorname{spt}^{*}(\mathcal{M})>k^{k+2}$ it now follows that there is $g \in \operatorname{Aut}(\mathcal{M})$ such that $d_{n}(g)=$ $\left|\operatorname{Spt}(g) \backslash \operatorname{Spt}\left(f_{0}, \ldots, f_{n}\right)\right|>0$. Hence there is also a maximal $f \in \operatorname{Aut}^{*}(\mathcal{M})$ such that $d_{n}(f)>0$. If we choose $f_{n+1} \in \operatorname{Aut}^{*}(\mathcal{M})$ so that $d_{n}\left(f_{n+1}\right)=\max _{g \in \operatorname{Aut}^{*}(\mathcal{M})} d_{n}(g)$, then $f_{0}, \ldots, f_{n+1}$ is a special sequence. This proves that there is a special sequence $f_{0}, \ldots, f_{l_{0}} \in \operatorname{Aut}^{*}(\mathcal{M})$ such that $d_{p}\left(f_{p+1}\right)>0$ for every $p=0, \ldots, l_{0}-1$. We fix this special sequence for the rest of the proof and use the abbreviations $\operatorname{Spt}(p)=\operatorname{Spt}\left(f_{p}\right)$ and $\operatorname{Spt}(0, \ldots, p)=\operatorname{Spt}\left(f_{0}, \ldots, f_{p}\right)$. 
We will prove that there are a subsequence (of distinct numbers) $t_{1}, \ldots, t_{k+1}$ of the sequence $0, \ldots, l_{0}$ and elements $b_{i} \in \operatorname{Spt}\left(f_{t_{i}}\right)$, for $i=1, \ldots, k+1$, such that $b_{i} \notin \operatorname{Spt}\left(f_{t_{j}}\right)$ if $j \neq i$; so $i \neq j$ implies $b_{i} \neq b_{j}$. Then $b_{1}, \ldots, b_{k+1} \in \operatorname{Spt}\left(f_{t_{1}} \circ \ldots \circ f_{t_{k+1}}\right)$, where of course the composition $f_{t_{1}} \circ \ldots \circ f_{t_{k+1}}$ belongs to $\operatorname{Aut}(\mathcal{M})$. This contradicts the assumption that $\mathcal{M} \in \mathbf{S}(\mathrm{spt} \leq k)$.

We will inductively define sequences $t_{0}^{i}, \ldots, t_{l_{i}}^{i}$, for $i=0, \ldots, k+1$, of indices from which we can extract a sequence $t_{1}, \ldots, t_{k+1}$ as above. Let $t_{j}^{0}=j$ for $j=0, \ldots, l_{0}=$ $k^{k+1}$. For each $p=2, \ldots, l_{0}$, there is, by Lemma 3.4, $a_{p} \in \operatorname{Spt}(1) \backslash \operatorname{Spt}(0)$ such that $a_{p} \notin \operatorname{Spt}(p)$. As $|\operatorname{Spt}(1)| \leq k$ there are $b_{1} \in \operatorname{Spt}(1) \backslash \operatorname{Spt}(0)$ and a subsequence of distinct numbers $t_{1}^{1}, \ldots, t_{l_{1}}^{1}$ of the sequence $2, \ldots, l_{0}$ such that, for all $p=t_{1}^{1}, \ldots, t_{l_{1}}^{1}$, $a_{p}=b_{1}$. Let $t_{1}=t_{0}^{1}=1$.

Now suppose that $m \leq k$ and that, for $i=1, \ldots, m, t_{0}^{i}, \ldots, t_{l_{i}}^{i}$ is a subsequence (of distinct numbers) of $t_{0}^{i-1}, \ldots, t_{l_{i-1}}^{i-1}, b_{i} \in \operatorname{Spt}\left(t_{0}^{i}\right) \backslash \operatorname{Spt}\left(0, \ldots, t_{0}^{i}-1\right)$ and $b_{i} \notin \operatorname{Spt}(p)$ for all $p=t_{1}^{i}, \ldots, t_{l_{i}}^{i}$. By Lemma 3.4, there is for each $p=t_{2}^{m}, \ldots, t_{l_{m}}^{m}$ an element $a_{p} \in \operatorname{Spt}\left(t_{1}^{m}\right) \backslash \operatorname{Spt}\left(0, \ldots, t_{1}^{m}-1\right)$ such that $a_{p} \notin \operatorname{Spt}(p)$. Since $\left|\operatorname{Spt}\left(t_{1}^{m}\right)\right| \leq k$ there are $b_{m+1} \in \operatorname{Spt}\left(t_{1}^{m}\right)$ and a subsequence $t_{1}^{m+1}, \ldots, t_{l_{m+1}}^{m+1}$ of $t_{2}^{m}, \ldots, t_{l_{m}}^{m}$ such that, for all $p=t_{1}^{m+1}, \ldots, t_{l_{m+1}}^{m+1}, a_{p}=b_{m+1}$. Let $t_{m+1}=t_{0}^{m+1}=t_{1}^{m}$. When $t_{0}^{i}, \ldots, t_{l_{i}}^{i}$ are defined for every $i=0, \ldots, k+1$ and $b_{i}$ for every $i=1, \ldots, k+1$, then, as already indicated, we take $t_{i}=t_{0}^{i}$ for $i=1, \ldots, k+1$.

Remark 3.6 Notice that the proofs up to now of this section do not need the assumption that we have considered a structure $\mathcal{M}$ and its automorphisms. We could, more generally, have considered a set $M$ and a group of permutations $H$ of $M$. If we do this, we get the following version of Proposition 3.5: If $k \geq 2$ is an integer and $H$ is a group of permutations of a set $M$ such that $\operatorname{spt}(h) \leq k$ for every $h \in H$, then

$$
\mid\{a \in M: h(a) \neq \text { a for some } h \in H\} \mid \leq k^{k+2} .
$$

Corollary 3.7 Let $m \in \mathbb{N}$. If $k=2 r(m !-1) m / m !+1$ and $T=k^{k+2}$ then

$$
\lim _{n \rightarrow \infty} \frac{\left|\mathbf{S}_{n}(\mathrm{spt} \geq m) \cap \mathbf{S}_{n}\left(\mathrm{spt}^{*} \leq T\right)\right|}{\left|\mathbf{S}_{n}(\mathrm{spt} \geq m)\right|}=\lim _{n \rightarrow \infty} \frac{\left|\mathbf{S}_{n}\left(\mathrm{spt}^{*} \geq m\right) \cap \mathbf{S}_{n}\left(\mathrm{spt}^{*} \leq T\right)\right|}{\left|\mathbf{S}_{n}\left(\mathrm{spt}^{*} \geq m\right)\right|}=1 .
$$

Proof Let $k=2 r(m !-1) m / m !+1$ and $T=k^{k+2}$. By Corollary 2.5,

$$
\left|\mathbf{S}_{n}(\mathrm{spt} \geq m)\right|=(1+o(1))\left|\mathbf{S}_{n}(m \leq \mathrm{spt} \leq k)\right|
$$

and by Proposition 3.5,

$$
\left|\mathbf{S}_{n}(m \leq \mathrm{spt} \leq k)\right|=\left|\mathbf{S}_{n}(m \leq \mathrm{spt} \leq k) \cap \mathbf{S}_{n}\left(\mathrm{spt}^{*} \leq T\right)\right|,
$$

so we get $\left|\mathbf{S}_{n}(\mathrm{spt} \geq m)\right|=(1+o(1))\left|\mathbf{S}_{n}(m \leq \mathrm{spt} \leq k) \cap \mathbf{S}_{n}\left(\mathrm{spt}^{*} \leq T\right)\right|$. The other limit is proved in the same way. 
Corollary 3.8 Suppose that $G$ is a finite group which is isomorphic to a group of permutations of $[m]$ where $m \in \mathbb{N}^{+}$. Then there is $T \in \mathbb{N}$, depending only on $G$ and the vocabulary, such that

$$
\lim _{n \rightarrow \infty} \frac{\mid\left\{\mathcal{M} \in \mathbf{S}_{n}: G \leq \operatorname{Aut}(\mathcal{M}) \text { and } \operatorname{spt}^{*}(\mathcal{M}) \leq T\right\} \mid}{\left|\left\{\mathcal{M} \in \mathbf{S}_{n}: G \leq \operatorname{Aut}(\mathcal{M})\right\}\right|}=1 .
$$

Proof By Corollary 2.6 we know that if $k=2 r(m !-1) m / m !+1$ then

$$
\lim _{n \rightarrow \infty} \frac{\left.\mid\left\{\mathcal{M} \in \mathbf{S}_{n}: G \leq \operatorname{Aut}(\mathcal{M}) \text { and } \operatorname{spt}(\mathcal{M}) \leq k\right)\right\} \mid}{\left|\left\{\mathcal{M} \in \mathbf{S}_{n}: G \leq \operatorname{Aut}(\mathcal{M})\right\}\right|}=1 .
$$

Let $T=k^{k+2}$. As Proposition 3.5 says that $\mathbf{S}_{n}(\mathrm{spt} \leq k) \subseteq \mathbf{S}_{n}\left(\mathrm{spt}^{*} \leq T\right)$ we are done.

\section{Asymptotic estimates of the number of structures with bounded support}

By Corollary 3.7, for arbitrary fixed $m \in \mathbb{N}$ and all large enough $n$, an overwhelming part of the members of $\mathbf{S}_{n}(\mathrm{spt} \geq m)$ belong $\mathbf{S}_{n}\left(\mathrm{spt}^{*} \leq T\right)$ for some $T$ depending only on $m$ and the vocabulary. We will show that an overwhelming part of the members of, for example, $\mathbf{S}_{n}$ (spt $\geq m$ ) for large enough $n$, belong to a finite union of sets of the form $\mathbf{S}_{n}(\mathcal{A}, H)$, defined below, where the structure $\mathcal{A}$ and permutation group $H$ depend only on the vocabulary and $m$. In order to understand the asymptotic behaviour of $\mathbf{S}_{n}(\mathrm{spt} \geq m)$ we will therefore, in this section, find asymptotic estimates of $\left|\mathbf{S}_{n}(\mathcal{A}, H)\right|$ as $n \rightarrow \infty$. As will become clear in the sequel, the sets of the form $\mathbf{S}_{n}(\mathcal{A}, H)$ are the "atomic" pieces of our analysis, and questions about, for example, $\mathbf{S}_{n}(\mathrm{spt} \geq m)$ or $\left\{\mathcal{M} \in \mathbf{S}_{n}: G \leq \operatorname{Aut}(\mathcal{M})\right\}$, for a fixed $G$, will be reduced to analysing quotients of the form $\left|\mathbf{S}_{n}\left(\mathcal{A}^{\prime}, H^{\prime}\right)\right| /\left|\mathbf{S}_{n}(\mathcal{A}, H)\right|$ as $n \rightarrow \infty$.

Recall that if $H$ is a group of permutations of $\Omega$ and $X \subseteq \Omega$ is the union of some of the orbits of $H$ on $\Omega$, then $H\lceil X=\{h \mid X: h \in H\}$ which is a permutation group on $X$. For every structure $\mathcal{M}, \operatorname{Spt}^{*}(\mathcal{M})$ is the union of all nonsingleton orbits of $\operatorname{Aut}(\mathcal{M})$ on $M$, so it always makes sense to speak about $\operatorname{Aut}(\mathcal{M})\left\lceil\operatorname{Spt}^{*}(\mathcal{M})\right.$ and we always have $\operatorname{Aut}(\mathcal{M})\left\lceil\operatorname{Spt}^{*}(\mathcal{M}) \cong \operatorname{Aut}(\mathcal{M})\right.$.

Definition 4.1 Let $\mathcal{A} \in \mathbf{S}$ be such that $\operatorname{Aut}(\mathcal{A})$ has no fixed point. Suppose that $H$ is a subgroup of $\operatorname{Aut}(\mathcal{A})$ such that $H$ has no fixed point. For each integer $n>0, \mathbf{S}_{n}(\mathcal{A}, H)$ is the set of $\mathcal{M} \in \mathbf{S}_{n}$ such that there is an isomorphism $f: \mathcal{A} \rightarrow \mathcal{M}\left\lceil\operatorname{Spt}^{*}(\mathcal{M})\right.$ such 
that $H_{f}=\left\{f \sigma f^{-1}: \sigma \in H\right\}$ is a subgroup of $\operatorname{Aut}(\mathcal{M})\left\lceil\operatorname{Spt}^{*}(\mathcal{M})\right.$. Note that $H_{f} \cong_{P} H$, so $\operatorname{Aut}(\mathcal{M})$ contains a copy of $H$ if $\mathcal{M} \in \mathbf{S}_{n}(\mathcal{A}, H)$. Let $\mathbf{S}(\mathcal{A}, H)=\bigcup_{n \in \mathbb{N}^{+}} \mathbf{S}_{n}(\mathcal{A}, H)$.

Lemma 4.2 Let $m \geq 2$ be an integer. There are $\mathcal{A}_{1}, \ldots, \mathcal{A}_{l} \in \mathbf{S}_{m}$ without any fixed point and, for each $i=1, \ldots, l$, subgroups $H_{i, 1}, \ldots, H_{i, l_{i}} \subseteq \operatorname{Aut}\left(\mathcal{A}_{i}\right)$ without any fixed point such that

$$
\mathbf{S}\left(\mathrm{spt}^{*}=m\right)=\bigcup_{i=1}^{l} \bigcup_{j=1}^{l_{i}} \mathbf{S}\left(\mathcal{A}_{i}, H_{i, j}\right)
$$

Proof Let $\mathcal{A}_{1}, \ldots, \mathcal{A}_{l}$ enumerate all structures of $\mathbf{S}_{m}$ that do not have any fixed point. Suppose that $\mathcal{M} \in \mathbf{S}\left(\operatorname{spt}^{*}=m\right)$. Then $\mathcal{M}\left\lceil\operatorname{Spt}^{*}(\mathcal{M}) \cong \mathcal{A}_{i}\right.$ for some $i$. If $K=\operatorname{Aut}(\mathcal{M})\left\lceil\operatorname{Spt}^{*}(\mathcal{M}), f: \mathcal{A}_{i} \rightarrow \mathcal{M}\left\lceil\operatorname{Spt}^{*}(\mathcal{M})\right.\right.$ is an isomorphism and $H=\left\{f^{-1} \sigma f:\right.$ $\sigma \in K\}$, then $H$ is a subgroup of $\operatorname{Aut}\left(\mathcal{A}_{i}\right)$ without any fixed point. From the definition of $\mathbf{S}\left(\mathcal{A}_{i}, H\right)$ it follows that $\mathcal{M} \in \mathbf{S}\left(\mathcal{A}_{i}, H\right)$. Hence every $\mathcal{M} \in \mathbf{S}\left(\mathrm{spt}^{*}=m\right)$ belongs to $\mathbf{S}\left(\mathcal{A}_{i}, H\right)$ for some $i$ and some subgroup $H \subseteq \operatorname{Aut}\left(\mathcal{A}_{i}\right)$. Conversely, for every $i=1, \ldots, l$ and every subgroup $H \subseteq \operatorname{Aut}\left(\mathcal{A}_{i}\right)$ we have $\mathbf{S}\left(\mathcal{A}_{i}, H\right) \subseteq \mathbf{S}\left(\mathrm{spt}^{*}=m\right)$, since $\operatorname{spt}^{*}(\mathcal{M})=m$ for every $\mathcal{M} \in \mathbf{S}\left(\mathcal{A}_{i}, H\right)$.

Lemma 4.3 (i) Let $m \geq 2$ be an integer. There are finitely many $\mathcal{A}_{1}, \ldots, \mathcal{A}_{l} \in \mathbf{S}$ without any fixed point and, for each $i=1, \ldots, l$, subgroups $H_{i, 1}, \ldots, H_{i, l_{i}} \subseteq \operatorname{Aut}\left(\mathcal{A}_{i}\right)$ without any fixed point such that

$$
\left|\mathbf{S}_{n}\left(\mathrm{spt}^{*} \geq m\right)\right| \sim\left|\bigcup_{i=1}^{l} \bigcup_{j=1}^{l_{i}} \mathbf{S}_{n}\left(\mathcal{A}_{i}, H_{i, j}\right)\right| \quad \text { as } n \rightarrow \infty .
$$

(ii) Part (ii) holds if ' $\mathrm{spt}^{*} \geq m$ ' is replaced by 'spt $\geq m$ '.

(iii) Let $G$ be a nontrivial finite group. There are finitely many $\mathcal{A}_{1}, \ldots, \mathcal{A}_{l} \in \mathbf{S}$ without any fixed point and, for each $i=1, \ldots, l$, subgroups $H_{i, 1}, \ldots, H_{i, l_{i}} \subseteq \operatorname{Aut}\left(\mathcal{A}_{i}\right)$ without any fixed point such that $G \leq H_{i, j}$ for all $i$ and $j$ and

$$
\left|\left\{\mathcal{M} \in \mathbf{S}_{n}: G \leq \operatorname{Aut}(\mathcal{M})\right\}\right| \sim\left|\bigcup_{i=1}^{l} \bigcup_{j=1}^{l_{i}} \mathbf{S}_{n}\left(\mathcal{A}_{i}, H_{i, j}\right)\right| \quad \text { as } n \rightarrow \infty .
$$

Proof (i) By Corollary 3.7, there is an integer $T$ such that

$$
\left|\mathbf{S}_{n}\left(\mathrm{spt}^{*} \geq m\right)\right| \sim\left|\mathbf{S}_{n}\left(m \leq \mathrm{spt}^{*} \leq T\right)\right| \quad \text { as } n \rightarrow \infty .
$$

Since $\mathbf{S}_{n}\left(m \leq \mathrm{spt}^{*} \leq T\right)=\bigcup_{i=m}^{T} \mathbf{S}_{n}\left(\mathrm{spt}^{*}=m\right)$, part (i) follows from Lemma 4.2.

(ii) By Corollary 3.7, there is $T$ such that

$$
\left|\mathbf{S}_{n}(\mathrm{spt} \geq m)\right| \sim\left|\mathbf{S}_{n}(\mathrm{spt} \geq m) \cap \mathbf{S}_{n}\left(\mathrm{spt}^{*} \leq T\right)\right| \quad \text { as } n \rightarrow \infty .
$$


As every $\mathcal{M} \in \mathbf{S}_{n}(\mathrm{spt} \geq m) \cap \mathbf{S}_{n}\left(\mathrm{spt}^{*} \leq T\right)$ belongs to $\mathbf{S}_{n}\left(\mathrm{spt}^{*}=p\right)$ for some $m \leq p \leq T$, we get (ii) from Lemma 4.2.

(iii) By Corollary 3.8, there is an integer $T$ such that

$$
\left|\left\{\mathcal{M} \in \mathbf{S}_{n}: G \leq \operatorname{Aut}(\mathcal{M})\right\}\right| \sim \mid\left\{\mathcal{M} \in \mathbf{S}_{n}: G \leq \operatorname{Aut}(\mathcal{M}) \text { and } \operatorname{spt}^{*}(\mathcal{M}) \leq T\right\} \mid
$$

as $n \rightarrow \infty$. Since every $\mathcal{M} \in\left\{\mathcal{M} \in \mathbf{S}_{n}: G \leq \operatorname{Aut}(\mathcal{M})\right.$ and $\left.\operatorname{spt}^{*}(\mathcal{M}) \leq T\right\}$ belongs to $\mathbf{S}_{n}\left(\right.$ spt $\left.^{*}=p\right)$ for some $p \leq T$, we also get part (iii) from Lemma 4.2 and its proof, which shows that we only need to consider $\mathcal{A}_{i}$ and $H_{i, j}$ such that $G \leq H_{i, j}$.

As suggested by the previous lemma, an essential step towards the main results is to asymptotically estimate $\left|\mathbf{S}_{n}(\mathcal{A}, H)\right|$ for any $\mathcal{A} \in \mathbf{S}$ without a fixed point and any subgroup $H \subseteq \operatorname{Aut}(\mathcal{A})$ without a fixed point.

Proposition 4.4 Suppose that $\mathcal{A} \in \mathbf{S}$ has no fixed point. Let $H$ be a subgroup of $\operatorname{Aut}(\mathcal{A})$ such that $H$ has no fixed point. Let $p=|A|$, for every $i=1, \ldots, r-1$ let $q_{i}$ be the number of orbits of $H$ on $A^{i}$ and, for every $i=1, \ldots r$, let $k_{i}$ be the number of relation symbols with arity $i$. There is an integer $c(\mathcal{A}, H)>0$, depending only on $\mathcal{A}$, $H$ and the vocabulary, such that

$$
\left|\mathbf{S}_{n}(\mathcal{A}, H)\right| \sim c(\mathcal{A}, H)\left(\begin{array}{l}
n \\
p
\end{array}\right) \exp _{2}\left(\sum_{i=1}^{r} k_{i}(n-p)^{i}+\sum_{j=2}^{r} \sum_{i=1}^{j-1} k_{j}\left(\begin{array}{l}
j \\
i
\end{array}\right) q_{i}(n-p)^{j-i}\right) .
$$

As will be explained below, Proposition 4.4 is a consequence of Lemma 4.6 which in turn follows from Lemmas 4.9-4.12.

Assumption 4.5 For the rest of this section we assume the following, although the assumptions may be restated:

Suppose that $\mathcal{A} \in \mathbf{S}$ has no fixed point.

Let $H$ be a subgroup of $\operatorname{Aut}(\mathcal{A})$ such that $H$ has no fixed point.

Also let

$$
p=|A|,
$$

for every $i=1, \ldots, r-1$ let $q_{i}$ be the number of orbits of $H$ on $A^{i}$ and,

for every $i=1, \ldots r$, let $k_{i}$ be the number of relation symbols with arity $i$. 
We consider the number of ways in which the relation symbols can be interpreted on $[n]$ so that the resulting structure belongs to $\mathbf{S}_{n}(\mathcal{A}, H)$. Let $c_{\mathcal{A}}$ be the number of structures in $\mathbf{S}_{p}$ that are isomorphic to $\mathcal{A}$. First, it is clear that we can choose the set $X \subseteq[n]$ which is going to be the support of the structure in $\left(\begin{array}{l}n \\ p\end{array}\right)$ ways, since we want that $|X|=p=|A|$. Then we can choose interpretations of the relation symbols on $X$ in $c_{\mathcal{A}}$ ways so that the resulting substructure with universe $X$, call it $\mathcal{A}_{X}$, is isomorphic to $\mathcal{A}$. Now suppose that $X \subseteq[n]$ of cardinality $p$ and $\mathcal{A}_{X} \cong \mathcal{A}$ with universe $X$ are fixed. Let

$$
\mathbf{S}_{n}\left(\mathcal{A}_{X}, H\right)=\left\{\mathcal{M} \in \mathbf{S}_{n}(\mathcal{A}, H): \mathcal{M}\left\lceil\operatorname{Spt}^{*}(\mathcal{M})=\mathcal{A}_{X}\right\} .\right.
$$

Note that the condition $\mathcal{M}\left\lceil\operatorname{Spt}^{*}(\mathcal{M})=\mathcal{A}_{X}\right.$ means that $\mathcal{M}\left\lceil\operatorname{Spt}^{*}(\mathcal{M})\right.$ is identical with $\mathcal{A}_{X}$. Also observe that if $X, X^{\prime} \subseteq[n]$ and $X \neq X^{\prime}$ then $\mathbf{S}_{n}\left(\mathcal{A}_{X}, H\right)$ and $\mathbf{S}_{n}\left(\mathcal{A}_{X^{\prime}}, H\right)$ are disjoint. Moreover, if both $\mathcal{A}_{X}^{\prime}$ and $\mathcal{A}_{X}$ have universe $X$ and are isomorphic with $\mathcal{A}$, but $\mathcal{A}_{X}^{\prime} \neq \mathcal{A}_{X}$, then $\mathbf{S}_{n}\left(\mathcal{A}_{X}, H\right)$ and $\mathbf{S}_{n}\left(\mathcal{A}_{X}^{\prime}, H\right)$ are disjoint. Therefore Proposition 4.4 follows from the following:

Lemma 4.6 Suppose that $X \subseteq[n]$ and $|X|=|A|=p$. There is an integer $d(\mathcal{A}, H)>0$, depending only on $\mathcal{A}, H$ and the vocabulary, such that

$$
\left|\mathbf{S}_{n}\left(\mathcal{A}_{X}, H\right)\right| \sim d(\mathcal{A}, H) \exp _{2}\left(\sum_{i=1}^{r} k_{i}(n-p)^{i}+\sum_{j=2}^{r} \sum_{i=1}^{j-1} k_{j}\left(\begin{array}{l}
j \\
i
\end{array}\right) q_{i}(n-p)^{j-i}\right) .
$$

Lemma 4.6 follows from Lemmas 4.9-4.12, as we will show after proving them. We begin with some preparatory work. Until Lemma 4.6 has been proved we fix $X \subseteq[n]$ such that $|X|=|A|=p$ and $\mathcal{A}_{X} \cong \mathcal{A}$ with universe $X$. For every isomorphism $f: \mathcal{A} \rightarrow \mathcal{A}_{X}$, let

$$
H_{f}=\left\{f \sigma f^{-1}: \sigma \in H\right\}
$$

so $H_{f}$ is a subgroup of $\operatorname{Aut}\left(\mathcal{A}_{X}\right)$ and $H_{f} \cong_{P} H$.

Suppose that $\mathcal{M} \in \mathbf{S}_{n}\left(\mathcal{A}_{X}, H\right)$. By the definition of $\mathbf{S}_{n}\left(\mathcal{A}_{X}, H\right), \mathcal{M}\left\lceil\operatorname{Spt}^{*}(\mathcal{M})=\mathcal{A}_{X}\right.$ and there is an isomorphism $f: \mathcal{A} \rightarrow \mathcal{A}_{X}$ such that $H_{f}$ is a subgroup of $\operatorname{Aut}(\mathcal{M})\left\lceil\operatorname{Spt}^{*}(\mathcal{M})\right.$. For each $t=1, \ldots, r-1$, the orbits of $H_{f}$ on $X^{t}$ forms a partition of $X^{t}$. If, for each $t=1, \ldots, r-1$, this partition is denoted $\Pi_{t}$, then since $\operatorname{Spt}^{*}(\mathcal{M})=X$, the following holds for $\mathcal{M}$ :

(a) Whenever $2 \leq j \leq r, R \in\left\{R_{1}, \ldots, R_{\rho}\right\}$ is a $j$-ary relation symbol, $1 \leq i<j$, $\left(a_{1}, \ldots, a_{i}\right) \in X^{i}$ and $\left(a_{1}^{\prime}, \ldots, a_{i}^{\prime}\right) \in X^{i}$ belong to the same part of $\Pi_{i}$ and $\left(a_{i+1}, \ldots, a_{j}\right)=\left(a_{i+1}^{\prime}, \ldots, a_{j}^{\prime}\right) \in([n] \backslash X)^{j-i}$, then for every $\pi \in S_{y m_{j}}$, either both of

$$
\left(a_{\pi(1)}, \ldots, a_{\pi(j)}\right) \text { and }\left(a_{\pi(1)}^{\prime}, \ldots, a_{\pi(j)}^{\prime}\right)
$$


or neither of them, belong to the interpretation of $R$.

Definition 4.7 If, for every $t=1, \ldots, r-1, \Pi_{t}$ is a partition of $X^{t}$ such that (a) holds (for $\mathcal{M}$ ), then we say that $\mathcal{M}$ respects (the sequence of partitions) $\Pi_{1}, \ldots, \Pi_{r-1}$.

Note that in the above definition there is no requirement that the partition $\Pi_{t}$ is the set of orbits of a permutation group.

Definition 4.8 A sequence $\Pi_{1}, \ldots, \Pi_{r-1}$ is called a sequence of $\left(\mathcal{A}_{X}, H\right)$-partitions if the following holds:

(b) there is an isomorphism $f: \mathcal{A} \rightarrow \mathcal{A}_{X}$ such that, for each $t=1, \ldots, r-1, \Pi_{t}$ is the set of orbits of $H_{f}$ on $X^{t}$.

We note the following: If $\mathcal{M} \in \mathbf{S}_{n}\left(\mathcal{A}_{X}, H\right)$ then $\mathcal{M}\left\lceil\operatorname{Spt}^{*}(\mathcal{M})=\mathcal{A}_{X}\right.$ and, for each $t=1, \ldots, r-1$, there is a partition $\Pi_{t}$ of $X^{t}$ such that $\mathcal{M}$ respects $\left(\Pi_{1}, \ldots, \Pi_{r-1}\right)$ and for some isomorphism $f: \mathcal{A} \rightarrow \mathcal{A}_{X}, \Pi_{t}$ is the set of orbits of $H_{f}$ on $X^{t}$ for $t=1, \ldots, r-1$. Conversely, if $\mathcal{M} \in \mathbf{S}_{n}$ is such that $\mathcal{M}\left\lceil\operatorname{Spt}^{*}(\mathcal{M})=\mathcal{A}_{X}\right.$ and, for each $t=1, \ldots, r-1$, there is a partition $\Pi_{t}$ of $X^{t}$ such that $\mathcal{M}$ respects $\left(\Pi_{1}, \ldots, \Pi_{r-1}\right)$ and for some isomorphism $f: \mathcal{A} \rightarrow \mathcal{A}_{X}, \Pi_{t}$ is the set of orbits of $H_{f}$ on $X^{t}$ for $t=1, \ldots, r-1$, then $H_{f}$ is a subgroup of $\mathcal{M}\left\lceil\operatorname{Spt}^{*}(\mathcal{M})\right.$ and therefore $\mathcal{M} \in \mathbf{S}_{n}\left(\mathcal{A}_{X}, H\right)$.

For every sequence of $\left(\mathcal{A}_{X}, H\right)$-partitions $\Pi_{1}, \ldots, \Pi_{r-1}$ we define

$$
\begin{aligned}
& \mathbf{S}_{n}\left(\mathcal{A}_{X}, \Pi_{1}, \ldots, \Pi_{r-1}\right)= \\
& \left\{\mathcal{M} \in \mathbf{S}_{n}: \mathcal{M}\left\lceil X=\mathcal{A}_{X}, \operatorname{Spt}^{*}(\mathcal{M})=X \text { and } \mathcal{M} \text { respects } \Pi_{1}, \ldots, \Pi_{r-1}\right\}\right.
\end{aligned}
$$

and

$$
\begin{aligned}
& \mathbf{T}_{n}\left(\mathcal{A}_{X}, \Pi_{1}, \ldots, \Pi_{r-1}\right)= \\
& \left\{\mathcal{M} \in \mathbf{S}_{n}: \mathcal{M}\left\lceil X=\mathcal{A}_{X} \text { and } \mathcal{M} \text { respects } \Pi_{1}, \ldots, \Pi_{r-1}\right\}\right.
\end{aligned}
$$

It follows directly from the definition that

$$
\mathbf{S}_{n}\left(\mathcal{A}_{X}, \Pi_{1}, \ldots, \Pi_{r-1}\right) \subseteq \mathbf{T}_{n}\left(\mathcal{A}_{X}, \Pi_{1}, \ldots, \Pi_{r-1}\right) .
$$

From the argument before the definition of $\mathbf{S}_{n}\left(\mathcal{A}_{X}, \Pi_{1}, \ldots, \Pi_{r-1}\right)$ it follows that

$$
\mathbf{S}_{n}\left(\mathcal{A}_{X}, H\right)=\bigcup_{\Pi_{1}, \ldots, \Pi_{r-1}} \mathbf{S}_{n}\left(\mathcal{A}_{X}, \Pi_{1}, \ldots, \Pi_{r-1}\right),
$$

where the union ranges over all sequences $\Pi_{1}, \ldots, \Pi_{r-1}$ of $\left(\mathcal{A}_{X}, H\right)$-partitions. The next step in the proof of Lemma 4.6 is to estimate $\left|\mathbf{S}_{n}\left(\mathcal{A}_{X}, \Pi_{1}, \ldots, \Pi_{r-1}\right)\right|$. Then we 
deal with the slightly problematic issue that even if $\Pi_{1}, \ldots, \Pi_{r-1}$ and $\Pi_{1}^{\prime}, \ldots, \Pi_{r-1}^{\prime}$ are different sequences of $\left(\mathcal{A}_{X}, H\right)$-partitions it may be the case that $\mathbf{S}\left(\mathcal{A}_{X}, \Pi_{1}, \ldots, \Pi_{r-1}\right)$ and $\mathbf{S}\left(\mathcal{A}_{X}, \Pi_{1}^{\prime}, \ldots, \Pi_{r-1}^{\prime}\right)$ have nonempty intersection. However, as we will show, their intersection will always be negligibly small, which implies that we can add the asymptotic estimates of the cardinalities of all $\mathbf{S}_{n}\left(\mathcal{A}_{X}, \Pi_{1}, \ldots, \Pi_{r-1}\right)$ to get an asymptotic estimate of the cardinality of $\mathbf{S}_{n}\left(\mathcal{A}_{X}, H\right)$. Recall that for $i=1, \ldots, r, k_{i}$ is the number of $i$-ary relation symbols. Also, $p=|A|=|X|$ and, for $i=1, \ldots, r-1, q_{i}$ is the number of orbits of $H$ on $A^{i}$.

Lemma 4.9 If $\Pi_{1}, \ldots, \Pi_{r-1}$ is a sequence of $\left(\mathcal{A}_{X}, H\right)$-partitions, then

$$
\left|\mathbf{T}_{n}\left(\mathcal{A}_{X}, \Pi_{1}, \ldots, \Pi_{r-1}\right)\right|=\exp _{2}\left(\sum_{i=1}^{r} k_{i}(n-p)^{i}+\sum_{j=2}^{r} \sum_{i=1}^{j-1} k_{j}\left(\begin{array}{l}
j \\
i
\end{array}\right) q_{i}(n-p)^{j-i}\right) .
$$

Moreover, there is $\varepsilon: \mathbb{N} \rightarrow \mathbb{R}$, depending only on $\mathcal{A}, H$ and the vocabulary, such that $\lim _{n \rightarrow \infty} \varepsilon(n)=0$ and for all large enough $n$ the proportion of $\mathcal{M} \in$ $\mathbf{T}_{n}\left(\mathcal{A}_{X}, \Pi_{1}, \ldots, \Pi_{r-1}\right)$ such that $\mathcal{M} \notin \mathcal{S}_{n}\left(\mathcal{A}_{X}, \Pi_{1}, \ldots, \Pi_{r-1}\right)$ is at most $\varepsilon(n)$.

Proof Suppose that $\Pi_{1}, \ldots, \Pi_{r-1}$ is a sequence of $\left(\mathcal{A}_{X}, H\right)$-partitions, so there is an isomorphism $f: \mathcal{A} \rightarrow \mathcal{A}_{X}$ such that, for each $t=1, \ldots, r-1, \Pi_{t}$ is the set of orbits of $H_{f}$ on $X^{t}$. Since $H_{f} \cong_{P} H$ it follows that $\Pi_{t}$ partitions $X^{t}$ into $q_{t}$ parts, for every $t=1, \ldots, r-1$. Let

$$
\gamma(n)=\exp _{2}\left(\sum_{i=1}^{r} k_{i}(n-p)^{i}+\sum_{j=2}^{r} \sum_{i=1}^{j-1} k_{j}\left(\begin{array}{l}
j \\
i
\end{array}\right) q_{i}(n-p)^{j-i}\right) .
$$

First we will prove that $\left|\mathbf{T}_{n}\left(\mathcal{A}_{X}, \Pi_{1}, \ldots, \Pi_{r-1}\right)\right|=\gamma(n)$. As observed before Lemma 4.9,

$$
\mathbf{S}_{n}\left(\mathcal{A}_{X}, \Pi_{1}, \ldots, \Pi_{r-1}\right) \subseteq \mathbf{T}_{n}\left(\mathcal{A}_{X}, \Pi_{1}, \ldots, \Pi_{r-1}\right)
$$

and $X \subseteq \operatorname{Spt}^{*}(\mathcal{M})$ for every $\mathcal{M} \in \mathbf{T}_{n}\left(\mathcal{A}_{X}, \Pi_{1}, \ldots, \Pi_{r-1}\right)$. Then we show that the proportion of $\mathcal{M} \in \mathbf{T}_{n}\left(\mathcal{A}_{X}, \Pi_{1}, \ldots, \Pi_{r-1}\right)$ such that $X$ is a proper subset of $\operatorname{Spt}^{*}(\mathcal{M})$ approaches 0 as $n \rightarrow \infty$. Moreover, we will get a bound $\varepsilon(n)$ as in the lemma. For the rest of the proof of this lemma we use the abbreviation

$$
\mathbf{T}_{n}=\mathbf{T}_{n}\left(\mathcal{A}_{X}, \Pi_{1}, \ldots, \Pi_{r-1}\right) .
$$

To determine $\left|\mathbf{T}_{n}\right|$ we consider the number of ways in which the relation symbols can be interpreted on $[n]$ so that the resulting structure $\mathcal{M}$ has the properties that $\mathcal{M} \uparrow X=\mathcal{A}_{X}$ and $\mathcal{M}$ respects $\Pi_{1}, \ldots, \Pi_{r-1}$, that is, (a) holds for $\mathcal{M}$. Since the substructure on $X$ must be $\mathcal{A}_{X}$, there is only one choice for the interpretations on tuples all of which coordinates belong to $X$. 
Now we consider in how many ways the relation symbols can be interpreted on tuples that intersect both $X$ and $[n] \backslash X$ so that resulting structure respects $\Pi_{1}, \ldots, \Pi_{r-1}$, so in this stage we only consider relation symbols of arity at least 2 . Let $R \in\left\{R_{1}, \ldots, R_{\rho}\right\}$ be a relation symbol of arity $j \geq 2$ and let $1 \leq i \leq j-1$. We consider the number of ways in which $R$ can be interpreted on $j$-tuples $\bar{a} \in[n]^{j}$ with exactly $i$ coordinates of $\bar{a}$ from $X$ in such a way that the resulting structure respects $\Pi_{1}, \ldots, \Pi_{r-1}$.

Suppose that

$$
a_{1}, \ldots, a_{i}, a_{1}^{\prime}, \ldots, a_{i}^{\prime} \in X \text { and } b_{i+1}, \ldots, b_{j} \in[n] \backslash X
$$

and that the $i$-tuples $\left(a_{1}, \ldots, a_{i}\right)$ and $\left(a_{1}^{\prime}, \ldots, a_{i}^{\prime}\right)$ belong to the same part of $\Pi_{i}$. Since we want (a) to be satisfied we have the choice of letting both $j$-tuples

$$
\left(a_{1}, \ldots, a_{i}, b_{i+1}, \ldots, b_{j}\right) \text { and }\left(a_{1}^{\prime}, \ldots, a_{i}^{\prime}, b_{i+1}, \ldots, b_{j}\right),
$$

or none of them, belong to the interpretation of $R$ (and this independently of other choices). We considered the case when $a_{1}, \ldots, a_{i}$ and $a_{1}^{\prime}, \ldots, a_{i}^{\prime}$ occurred in the first $i$ positions of the respective $j$-tuple, but the same is clearly true if $a_{1}, \ldots, a_{i}$ and $a_{1}^{\prime}, \ldots, a_{i}^{\prime}$ take other positions in the respective $j$-tuples, but still so that $a_{l}$ precedes $a_{l^{\prime}}$ if $l<l^{\prime}$ and $a_{l}$ takes position $t$ if and only if $a_{l}^{\prime}$ takes position $t .^{5}$ There are $\left(\begin{array}{l}j \\ i\end{array}\right)$ ways in which $i$ positions in an $j$-tuple can be chosen. Therefore the number of ways to choose the interpretation of $R$ on $j$-tuples with exactly $i$ coordinates in $X$ in such a way that (a) is satisfied is

$$
\exp _{2}\left(\left(\begin{array}{l}
j \\
i
\end{array}\right) q_{i}(n-p)^{j-i}\right)
$$

where we recall that $q_{i}$ is the number of parts of the partition $\Pi_{i}$ of $X^{i} .^{6}$ If $i^{\prime} \neq i$ and $1 \leq i^{\prime} \leq j-1$ then the corresponding number of choices for $j$-tuples with exactly $i^{\prime}$ coordinates in $X$ is independent from the previously made choices. Therefore the number of ways in which $R$ can be interpreted on tuples that intersect both $X$ and $[n] \backslash X$ is

$$
\exp _{2}\left(\sum_{i=1}^{j-1}\left(\begin{array}{l}
j \\
i
\end{array}\right) q_{i}(n-p)^{j-i}\right) .
$$

The same argument can be carried out for every relation symbol $R$ of arity at least 2 . The number of choices for each such $R$ is independent of previously made choices.

\footnotetext{
${ }^{5}$ We consider only the given order of $a_{1}, \ldots, a_{i}$ and $a_{1}^{\prime}, \ldots, a_{i}^{\prime}$ because, in general, an $i$-tuple obtained by reordering $a_{1}, \ldots, a_{i}$ need not belong to the same part of $\Pi_{i}$ as $\left(a_{1}, \ldots, a_{i}\right)$.

${ }^{6}$ If we assume that $R$ is always interpreted as an irreflexive and symmetric relation, then the corresponding number is $\exp _{2}\left(q_{i}^{\prime}\left(\begin{array}{c}n-p \\ j-i\end{array}\right)\right)$ where $q_{i}^{\prime}$ is the number of orbits of the action of $H$ on $\{B \subseteq A:|B|=i\}$ given by $h\left(\left\{b_{1}, \ldots, b_{i}\right\}\right)=\left\{h\left(b_{1}\right), \ldots, h\left(b_{i}\right)\right\}$ for every $h \in H$ and $i$-subset $\left\{b_{1}, \ldots, b_{i}\right\}$ of $A$.
} 
Therefore the number of ways in which all relation symbols with arity at least 2 can be interpreted on tuples that intersect both $X$ and $[n] \backslash X$ in such a way that (a) is satisfied is

$$
\exp _{2}\left(\sum_{j=2}^{r} \sum_{i=1}^{j-1} k_{j}\left(\begin{array}{l}
j \\
i
\end{array}\right) q_{i}(n-p)^{j-i}\right)
$$

Finally we consider interpretations on tuples $\bar{a}$ such that none of the coordinates of $\bar{a}$ belongs to $X$. If $R$ has arity $i$, then there are $2^{(n-p)^{i}}$ ways in which to interpret $R$ on tuples $\bar{a} \in([n] \backslash X)^{i}$, independently of other choices. As there are $k_{i}$ relation symbols of arity $i$, the number of ways to interpret all relation symbols on $[n] \backslash X$ is

$$
\exp _{2}\left(\sum_{i=1}^{r} k_{i}(n-p)^{i}\right)
$$

Suppose that a structure $\mathcal{M}$ has been constructed by making the choices described above. Then, by construction, $\mathcal{M}\left\lceil X=\mathcal{A}_{X}\right.$ and $\mathcal{M}$ respects $\Pi_{1}, \ldots, \Pi_{r-1}$. By assumption, $H$ has no fixed point which implies that every part of the partition $\Pi_{1}$ of $X$ has at least two members. Since $\mathcal{M}$ respects $\Pi_{1}, \ldots, \Pi_{r-1}$ and $\Pi_{1}, \ldots, \Pi_{r-1}$ is a sequence of $\left(\mathcal{A}_{X}, H\right)$-partitions it follows that $X \subseteq \operatorname{Spt}^{*}(\mathcal{M})$. It is also clear that every member of $\mathbf{T}_{n}$ can be obtained in exactly one way by making choices as described by the construction. Hence, by multiplying (4-6) and (4-5), we see that $\left|\mathbf{T}_{n}\right|=\gamma(n)$.

It remains to prove that for all large enough $n$,

$$
\frac{\left|\left\{\mathcal{M} \in \mathbf{T}_{n}: \operatorname{Spt}^{*}(\mathcal{M}) \neq X\right\}\right|}{\left|\mathbf{T}_{n}\right|} \leq \varepsilon(n),
$$

where $\lim _{n \rightarrow \infty} \varepsilon(n)=0$ and $\varepsilon$ depends only on $\mathcal{A}, H$ and the vocabulary. After defining $\mathbf{T}_{n}=\mathbf{T}_{n}\left(\mathcal{A}_{X}, \Pi_{1}, \ldots, \Pi_{r-1}\right)$, see (4-3), we observed that if $\mathcal{M} \in \mathbf{T}_{n}$ then $X \subseteq \operatorname{Spt}^{*}(\mathcal{M})$. Since $\Pi_{1}, \ldots, \Pi_{r-1}$ is a sequence of $\left(\mathcal{A}_{X}, H\right)$-partitions, there is an isomorphism $f: \mathcal{A} \rightarrow \mathcal{A}_{X}$ such that, for each $t=1, \ldots, r-1, \Pi_{t}$ is the set of orbits of $H_{f}=\left\{f \sigma f^{-1}: \sigma \in H\right\}$ on $X^{t}$. Let $H_{f}=\left\{h_{1}, \ldots, h_{s}\right\}$ and extend every $h_{i} \in H_{f}$ to $h_{i}^{\prime} \in \operatorname{Sym}_{n}$ by $h_{i}^{\prime}(x)=h_{i}(x)$ if $x \in X$ and $h_{i}^{\prime}(x)=x$ if $x \in[n] \backslash X$. Then $\operatorname{Spt}\left(h_{1}^{\prime}, \ldots, h_{s}^{\prime}\right)=X$ and hence $\operatorname{spt}\left(h_{1}^{\prime}, \ldots, h_{s}^{\prime}\right)=|X|=|A|=p$.

If $\mathcal{M} \in \mathbf{S}_{n}$, then $\mathcal{M}$ belongs to $\mathbf{S}_{n}\left(h_{1}^{\prime}, \ldots, h_{s}^{\prime}\right)$ if and only if the following condition holds: for every $t=1, \ldots, r$ and every $t$-ary relation symbol $R$, if $\bar{a}$ and $\bar{b}$ are two $t$ tuples from the same orbit of $\left\langle h_{1}^{\prime}, \ldots, h_{s}^{\prime}\right\rangle$ on $[n]^{t}$ (which here denotes the set of ordered $t$-tuples of elements from $[n]$ ), then either $\mathcal{M} \models R(\bar{a}) \wedge R(\bar{b})$ or $\mathcal{M} \models \neg R(\bar{a}) \wedge \neg R(\bar{b})$. As $X$ is a union of orbits of $\left\langle h_{1}^{\prime}, \ldots, h_{s}^{\prime}\right\rangle$ it follows that if we define

$$
\mathbf{S}_{n}\left(h_{1}^{\prime}, \ldots, h_{s}^{\prime}, \mathcal{A}_{X}\right)=\left\{\mathcal{M} \in \mathbf{S}_{n}\left(h_{1}^{\prime}, \ldots, h_{s}^{\prime}\right): \mathcal{M}\left\lceil X=\mathcal{A}_{X}\right\}\right.
$$


then there is a constant $0<c \leq 1$, depending only on $\mathcal{A}, H$ and the vocabulary, such that

$$
\left|\mathbf{S}_{n}\left(h_{1}^{\prime}, \ldots, h_{s}^{\prime}, \mathcal{A}_{X}\right)\right|=c\left|\mathbf{S}_{n}\left(h_{1}^{\prime}, \ldots, h_{s}^{\prime}\right)\right| .
$$

From the definition of $h_{1}^{\prime}, \ldots, h_{s}^{\prime}$ it follows that

$$
\mathbf{S}_{n}\left(h_{1}^{\prime}, \ldots, h_{s}^{\prime}, \mathcal{A}_{X}\right) \subseteq \mathbf{T}_{n} .
$$

By (4-8), (4-9) and Propositions 2.3 and 3.5, there are $\lambda, p_{0}>0$, depending only on $\mathcal{A}, H$ and the vocabulary, such that for all sufficiently large $n$,

$$
\frac{\left|\mathbf{S}_{n}\left(\mathrm{spt}^{*}>p_{0}\right)\right|}{\left|\mathbf{T}_{n}\right|} \leq \frac{\left|\mathbf{S}_{n}\left(\mathrm{spt}^{*}>p_{0}\right)\right|}{c\left|\mathbf{S}_{n}\left(h_{1}^{\prime}, \ldots, h_{s}^{\prime}\right)\right|} \leq 2^{-\lambda n^{r-1}} .
$$

Hence, for all large enough $n$, the proportion of $\mathcal{M} \in \mathbf{T}_{n}$ such that $\operatorname{spt}^{*}(\mathcal{M}) \leq p_{0}$ is at least $1-2^{-\lambda n^{r-1}}$.

Fix any $a \in[n] \backslash X$ and $a^{\prime} \in[n]$ such that $a \neq a^{\prime}$. From the definition of $\mathbf{T}_{n}$ it is clear that for every sequence of distinct $(r-1)$-tuples $\bar{b}_{1}, \ldots, \bar{b}_{\kappa} \in\left([n] \backslash\left(X \cup\left\{a, a^{\prime}\right\}\right)\right)^{r-1}$, the proportion of $\mathcal{M} \in \mathbf{T}_{n}$ that satisfies the following is $2^{-\kappa}$ :

$$
\text { for every } i=1, \ldots, \kappa, \mathcal{M} \models R\left(a, \bar{b}_{i}\right) \Longleftrightarrow \mathcal{M}=R\left(a^{\prime}, \bar{b}_{i}\right) \text {. }
$$

Observe that if $\mathcal{M} \in \mathbf{T}_{n}, \operatorname{spt}^{*}(\mathcal{M}) \leq p_{0}$ and $g(a)=a^{\prime}$ for some $g \in \operatorname{Aut}(\mathcal{M})$, then there is a sequence of distinct $(r-1)$-tuples $\bar{b}_{1}, \ldots, \bar{b}_{\kappa} \in\left([n] \backslash\left(X \cup\left\{a, a^{\prime}\right\}\right)\right)^{r-1}$ such that $\kappa=2^{\left(n-p_{0}-2\right)^{r-1}}$ and (4-10) is satisfied. Hence the proportion of $\mathcal{M} \in \mathbf{T}_{n}$ such that $\operatorname{spt}^{*}(\mathcal{M}) \leq p_{0}$ and $g(a)=a^{\prime}$ for some $g \in \operatorname{Aut}(\mathcal{M})$ is at most $2^{-\left(n-p_{0}-2\right)^{r-1}}$. As the proportion of $\mathcal{M} \in \mathbf{T}_{n}$ such that $\operatorname{spt}^{*}(\mathcal{M}) \leq p_{0}$ is at least $1-2^{-\lambda n^{r-1}}$, it follows that the proportion of $\mathcal{M} \in \mathbf{T}_{n}$ with an automorphism $g$ such that $g(a)=a^{\prime}$ is at most $2^{-\left(n-p_{0}-2\right)^{r-1}}+2^{-\lambda n^{r-1}}$. It follows that the proportion of $\mathcal{M} \in \mathbf{T}_{n}$ which have distinct elements $a \in[n] \backslash X$ and $a^{\prime} \in[n]$ and an automorphism $g$ such that $g(a)=a^{\prime}$ is at most $n^{2}\left(2^{-\left(n-p_{0}-2\right)^{r-1}}+2^{-\lambda n^{r-1}}\right)$. This immediately implies (4-7), so the proof of Lemma 4.9 is finished.

Remark 4.10 If we assume that all relation symbols are always interpreted as irreflexive and symmetric relations then we get

$$
\left|\mathbf{T}_{n}\left(\mathcal{A}_{X}, \Pi_{1}, \ldots, \Pi_{r-1}\right)\right|=\exp _{2}\left(\sum_{i=1}^{r} k_{i}\left(\begin{array}{c}
n-p \\
i
\end{array}\right)+\sum_{j=2}^{r} \sum_{i=1}^{j-1} k_{j} q_{i}^{\prime}\left(\begin{array}{c}
n-p \\
j-i
\end{array}\right)\right),
$$

where $q_{i}^{\prime}$ is the number of orbits of the action of $H$ on $\{B \subseteq A:|B|=i\}$ given by $h\left(\left\{b_{1}, \ldots, b_{i}\right\}\right)=\left\{h\left(b_{1}\right), \ldots, h\left(b_{i}\right)\right\}$ for every $h \in H$ and $i$-subset $\left\{b_{1}, \ldots, b_{i}\right\}$ of $A$. Under the same assumptions we still have

$$
\left|\mathbf{S}_{n}\left(\mathcal{A}_{X}, \Pi_{1}, \ldots, \Pi_{r-1}\right)\right| \sim\left|\mathbf{T}_{n}\left(\mathcal{A}_{X}, \Pi_{1}, \ldots, \Pi_{r-1}\right)\right|
$$


by the same argument as above (and a modification of Proposition 2.3).

Lemma 4.11 Suppose that $\Pi_{1}, \ldots, \Pi_{r-1}$ is a sequence of $\left(\mathcal{A}_{X}, H\right)$-partitions. For each $1 \leq i<r$, the proportion of $\mathcal{M} \in \mathbf{S}_{n}\left(\mathcal{A}_{X}, \Pi_{1}, \ldots, \Pi_{r-1}\right)$ with the following property is at most $\varepsilon(n)$ where $\varepsilon(n) \rightarrow 0$ as $n \rightarrow 0$ and the function $\varepsilon$ depends only on $\mathcal{A}, H$ and the vocabulary:

(†) There are an $r$-ary relation symbol $R$, different parts $P, P^{\prime} \in \Pi_{i}, \bar{a}=$ $\left(a_{1}, \ldots, a_{i}\right) \in P$ and $\bar{a}^{\prime}=\left(a_{1}^{\prime}, \ldots, a_{i}^{\prime}\right) \in P^{\prime}$ such that for every $\bar{b}=\left(b_{i+1}, \ldots, b_{r}\right) \in([n] \backslash X)^{r-i}$,

$$
\mathcal{M} \models R(\bar{a}, \bar{b}) \Longleftrightarrow \mathcal{M} \models R\left(\bar{a}^{\prime}, \bar{b}\right) .
$$

Proof By Lemma 4.9 it suffices to prove that the proportion of

$\mathcal{M} \in \mathbf{T}_{n}\left(\mathcal{A}_{X}, \Pi_{1}, \ldots, \Pi_{r-1}\right)$ with property ( $\left.\dagger\right)$ is at most $\varepsilon(n)$ where $\varepsilon(n) \rightarrow 0$ as $n \rightarrow 0$ and $\varepsilon$ depends only on $\mathcal{A}, H$ and the vocabulary. Suppose that $\mathcal{M} \in \mathbf{T}_{n}\left(\mathcal{A}_{X}, \Pi_{1}, \ldots, \Pi_{t}\right)$ and $(\dagger)$ holds, so there are different parts $P, P^{\prime} \in \Pi_{i}, \bar{a}=\left(a_{1}, \ldots, a_{i}\right) \in P$ and $\bar{a}^{\prime}=\left(a_{1}^{\prime}, \ldots, a_{i}^{\prime}\right) \in P^{\prime}$ such that for every $\bar{b}=\left(b_{i+1}, \ldots, b_{r}\right) \in([n] \backslash X)^{r-i}$,

$$
\mathcal{M} \models R(\bar{a}, \bar{b}) \Longleftrightarrow \mathcal{M} \models R\left(\bar{a}^{\prime}, \bar{b}\right) .
$$

Fix these tuples $\bar{a}$ and $\bar{a}^{\prime}$. The number of ways in which we can interpret $R$, in such $\mathcal{M}$, on tuples of the form $\bar{a} \bar{b}$ and $\bar{a}^{\prime} \bar{b}$ where $\bar{b} \in([n] \backslash X)^{r-i}$ is $2^{(n-p)^{r-i}}$, independently of how $R$ is interpreted on other tuples and independently of how other relation symbols are interpreted.

On the other hand, for $\mathcal{M} \in \mathbf{T}_{n}\left(\mathcal{A}_{X}, \Pi_{1}, \ldots, \Pi_{r-1}\right)$ without property $(\dagger)$, the number of ways in which $R$ can be interpreted on tuples of the form $\bar{a} \bar{b}$ and $\bar{a}^{\prime} \bar{b}$ where $\bar{b} \in([n] \backslash X)^{r-i}$ is $4^{(n-p)^{r-i}}$, independently of how $R$ is interpreted on other tuples and independently of how other relation symbols are interpreted. Therefore the proportion of $\mathcal{M} \in \mathbf{T}_{n}\left(\mathcal{A}_{X}, \Pi_{1}, \ldots, \Pi_{r-1}\right)$ with property $(\dagger)$ is at most $2^{(n-p)^{r-i}} / 4^{(n-p)^{r-i}} \leq$ $2^{-(n-p)}$.

Lemma 4.12 If $\Pi_{1}, \ldots, \Pi_{r-1}$ and $\Pi_{1}^{\prime}, \ldots, \Pi_{r-1}^{\prime}$ are two different sequences of $\left(\mathcal{A}_{X}, H\right)$-partitions, then

$$
\frac{\left|\mathbf{S}_{n}\left(\mathcal{A}_{X}, \Pi_{1}, \ldots, \Pi_{r-1}\right) \cap \mathbf{S}_{n}\left(\mathcal{A}_{X}, \Pi_{1}^{\prime}, \ldots, \Pi_{r-1}^{\prime}\right)\right|}{\left|\mathbf{S}_{n}\left(\mathcal{A}_{X}, \Pi_{1}, \ldots, \Pi_{r-1}\right) \cup \mathbf{S}_{n}\left(\mathcal{A}_{X}, \Pi_{1}^{\prime}, \ldots, \Pi_{r-1}^{\prime}\right)\right|} \leq \varepsilon(n) .
$$

where $\varepsilon(n) \rightarrow 0$ as $n \rightarrow 0$ and the function $\varepsilon$ depends only on $\mathcal{A}, H$ and the vocabulary. 
Proof Suppose that $\Pi_{1}, \ldots, \Pi_{r-1}$ and $\Pi_{1}^{\prime}, \ldots, \Pi_{r-1}^{\prime}$ are different sequences of $\left(\mathcal{A}_{X}, H\right)$-partitions and that

$$
\mathcal{M} \in \mathbf{S}_{n}\left(\mathcal{A}_{X}, \Pi_{1}, \ldots, \Pi_{r-1}\right) \cap \mathbf{S}_{n}\left(\mathcal{A}_{X}, \Pi_{1}^{\prime}, \ldots, \Pi_{r-1}^{\prime}\right) .
$$

Then for some $1 \leq i<r$, there are $\bar{a}, \bar{a}^{\prime} \in X^{i}$ such that $\bar{a}$ and $\bar{a}^{\prime}$ are in the same part of the partition $\Pi_{i}^{\prime}$ but in different parts of the partition $\Pi_{i}$, or vice versa. In the first case, $\mathcal{M}$ has property $(\dagger$ ) from Lemma 4.11 (for every $r$-ary relation symbol $R$ ) when seen as a member of $\mathbf{S}_{n}\left(X, \Pi_{1}, \ldots, \Pi_{r-1}\right)$. In the second case, $\mathcal{M}$ has property ( $\dagger$ ) when seen as a member of $\mathbf{S}_{n}\left(X, \Pi_{1}^{\prime}, \ldots, \Pi_{r-1}^{\prime}\right)$. Therefore, using Lemma 4.11, the quotient of the lemma is at most $2 \varepsilon(n)$ where $\varepsilon(n) \rightarrow 0$ as $n \rightarrow 0$ and the function $\varepsilon$ depends only on $\mathcal{A}, H$ and the vocabulary..

Proof of Lemma 4.6. Let

$$
\gamma(n)=\exp _{2}\left(\sum_{i=1}^{r} k_{i}(n-p)^{i}+\sum_{j=2}^{r} \sum_{i=1}^{j-1} k_{j}\left(\begin{array}{l}
j \\
i
\end{array}\right) q_{i}(n-p)^{j-i}\right)
$$

and let $d(\mathcal{A}, H)$ be the number of different sequences $\Pi_{1}, \ldots, \Pi_{r-1}$ of $\left(\mathcal{A}_{X}, H\right)$ partitions. Hence, $d(\mathcal{A}, H)$ is finite and depends only on $\mathcal{A}, H$ and the vocabulary. We prove that $\left|\mathbf{S}_{n}\left(\mathcal{A}_{X}, H\right)\right| \sim d(\mathcal{A}, H) \gamma(n)$. From (4-4) it follows that

$$
\left|\mathbf{S}_{n}\left(\mathcal{A}_{X}, H\right)\right| \leq d(\mathcal{A}, H) \gamma(n) .
$$

Let $\mathbf{U}_{n}$ be the union of all intersections

$$
\mathbf{S}_{n}\left(\mathcal{A}_{X}, \Pi_{1}, \ldots, \Pi_{r-1}\right) \cap \mathbf{S}_{n}\left(\mathcal{A}_{X}, \Pi_{1}^{\prime}, \ldots, \Pi_{r-1}^{\prime}\right)
$$

where $\Pi_{1}, \ldots, \Pi_{r-1}$ and $\Pi_{1}^{\prime}, \ldots, \Pi_{r-1}^{\prime}$ range over all unordered pairs of different sequences of $\left(\mathcal{A}_{X}, H\right)$-partitions. If the sums below ranges over such unordered pairs, then, by Lemma 4.12, we have

$$
\begin{aligned}
\left|\mathbf{U}_{n}\right| & \leq \sum\left|\mathbf{S}_{n}\left(\mathcal{A}_{X}, \Pi_{1}, \ldots, \Pi_{r-1}\right) \cap \mathbf{S}_{n}\left(\mathcal{A}_{X}, \Pi_{1}^{\prime}, \ldots, \Pi_{r-1}^{\prime}\right)\right| \\
& \leq \varepsilon(n) \sum\left(\left|\mathbf{S}_{n}\left(\mathcal{A}_{X}, \Pi_{1}, \ldots, \Pi_{r-1}\right)\right|+\left|\mathbf{S}_{n}\left(\mathcal{A}_{X}, \Pi_{1}^{\prime}, \ldots, \Pi_{r-1}^{\prime}\right)\right|\right) \\
& \sim \varepsilon(n) \cdot\left(\begin{array}{c}
d(\mathcal{A}, H) \\
2
\end{array}\right) \cdot 2 \gamma(n),
\end{aligned}
$$

where $\varepsilon(n) \rightarrow 0$ as $n \rightarrow \infty$. By Lemma $4.9, \mathbf{S}_{n}\left(\mathcal{A}_{X}, \Pi_{1}, \ldots, \Pi_{r-1}\right) \sim \gamma(n)$ for every sequence $\Pi_{1}, \ldots, \Pi_{r-1}$ of $\left(\mathcal{A}_{X}, H\right)$-partitions. It follows that, for every such sequence,

$$
\left|\mathbf{S}_{n}\left(\mathcal{A}_{X}, \Pi_{1}, \ldots, \Pi_{r-1}\right)\right|-\left|\mathbf{U}_{n}\right| \sim \gamma(n) .
$$


Since $\mathbf{S}_{n}\left(\mathcal{A}_{X}, \Pi_{1}, \ldots, \Pi_{r-1}\right) \backslash \mathbf{U}_{n}$ and $\mathbf{S}_{n}\left(\mathcal{A}_{X}, \Pi_{1}^{\prime}, \ldots, \Pi_{r-1}^{\prime}\right) \backslash \mathbf{U}_{n}$ are disjoint if $\Pi_{1}, \ldots, \Pi_{r-1}$ and $\Pi_{1}^{\prime}, \ldots, \Pi_{r-1}^{\prime}$ are different sequences, it follows that

$$
\begin{aligned}
\left|\mathbf{S}_{n}\left(\mathcal{A}_{X}, H\right)\right| & \geq \sum\left|\mathbf{S}_{n}\left(\mathcal{A}_{X}, \Pi_{1}, \ldots, \Pi_{r-1}\right) \backslash \mathbf{U}_{n}\right| \\
& \geq \sum\left(\left|\mathbf{S}_{n}\left(\mathcal{A}_{X}, \Pi_{1}, \ldots, \Pi_{r-1}\right)\right|-\left|\mathbf{U}_{n}\right|\right) \sim d(\mathcal{A}, H) \gamma(n),
\end{aligned}
$$

where the sums range over all sequences $\Pi_{1}, \ldots, \Pi_{r-1}$ of $\left(\mathcal{A}_{X}, H\right)$-partitions. This together with (4-11) implies that $\left|\mathbf{S}_{n}\left(\mathcal{A}_{X}, H\right)\right| \sim d(\mathcal{A}, H) \gamma(n)$, so Lemma 4.6 is proved

As explained in the paragraph after the statement of Proposition 4.4, it follows from Lemma 4.6, so now we have also proved Proposition 4.4. We can now derive two corollaries of this proposition. These corollaries, as well as the proposition itself will be used in the next section. It will be convenient to use the following notation:

Definition 4.13 Suppose that $H$ is a group of permutations of the set $\Omega$. Then $p(H)=|\Omega|, q(H)$ is the number of orbits of $H$ on $\Omega$ and $s(H)$ is the number of orbits of $H$ on $\Omega^{2}$.

Corollary 4.14 Suppose that $r=2$, that $\mathcal{A} \in \mathbf{S}$ has no fixed point and let $H$ be a subgroup of $\operatorname{Aut}(\mathcal{A})$ without any fixed point. Let $p=p(H)=|A|$, let $q=q(H)$ and for $i=1,2$ let $k_{i}$ be the number of relation symbols of arity $i$. Then there is an integer $c(\mathcal{A}, H)>0$, depending only on $\mathcal{A}, H$ and the vocabulary, such that

$$
\left|\mathbf{S}_{n}(\mathcal{A}, H)\right| c(\mathcal{A}, H)\left(\begin{array}{l}
n \\
p
\end{array}\right) \exp _{2}\left(k_{2} n^{2}-2 k_{2}(p-q) n+k_{1} n+k_{2} p^{2}-k_{1} p\right) .
$$

Proof By Proposition 4.4 with $r=2$ and $q=q_{1}$, there is an integer $c(\mathcal{A}, H)>0$, depending only on $\mathcal{A}, H$ and the vocabulary, such that

$$
\begin{aligned}
\left|\mathbf{S}_{n}(\mathcal{A}, H)\right| & \sim c(\mathcal{A}, H)\left(\begin{array}{l}
n \\
p
\end{array}\right) \exp _{2}\left(\sum_{i=1}^{2} k_{i}(n-p)^{i}+2 k_{2} q(n-p)\right) \\
& =c(\mathcal{A}, H)\left(\begin{array}{l}
n \\
p
\end{array}\right) \exp _{2}\left(k_{2} n^{2}-2 k_{2}(p-q) n+k_{1} n+k_{2} p^{2}-k_{1} p\right) .
\end{aligned}
$$

Corollary 4.15 Suppose that $r>2$, that $\mathcal{A} \in \mathbf{S}$ has no fixed point and that $H$ is a subgroup of $\operatorname{Aut}(\mathcal{A})$ without any fixed point. Let $p=p(H)=|A|$, let $q=q(H)$ and $s=s(H)$. Moreover, let $k$ be the number of $r$-ary relation symbols, let $l$ be the number 
of $(r-1)$-ary relation symbols, let $m$ be the number of $(r-2)$-ary relation symbols and define

$$
\beta(x, y, z)=k\left(\begin{array}{l}
r \\
2
\end{array}\right) x^{2}-k r(r-1) x y-l(r-1) x+l(r-1) y+k\left(\begin{array}{l}
r \\
2
\end{array}\right) z .
$$

Then there is an integer $c(\mathcal{A}, H)$, depending only on $\mathcal{A}, H$ and the vocabulary, such that

$$
\begin{aligned}
\left|\mathbf{S}_{n}(\mathcal{A}, H)\right| \sim c(\mathcal{A}, H)\left(\begin{array}{l}
n \\
p
\end{array}\right) \exp _{2}\left(k n^{r}-(k r(p-q)-l) n^{r-1}\right. & \\
& \left.+(\beta(p, q, s)+m) n^{r-2}+\mathcal{O}\left(n^{r-3}\right)\right) .
\end{aligned}
$$

Proof For every $i=1, \ldots r-1$, let $q_{i}$ be the number of orbits of $H$ on $A^{i}$. For every $j=1, \ldots, r$, let $k_{j}$ be the number of relation symbols of arity $j$. So we have $q_{1}=q, q_{2}=s, k_{r}=k, k_{r-1}=l$ and $k_{r-2}=m$. By Proposition 4.4, there is an integer $c(\mathcal{A}, H)>0$, depending only on $\mathcal{A}, H$ and the vocabulary, such that

$$
\left|\mathbf{S}_{n}(\mathcal{A}, H)\right| \sim c(\mathcal{A}, H)\left(\begin{array}{l}
n \\
p
\end{array}\right) \exp _{2}(\lambda(n)),
$$

where

$$
\begin{aligned}
\lambda(n) & =\sum_{i=1}^{r} k_{i}(n-p)^{i}+\sum_{j=2}^{r} \sum_{i=1}^{j-1} k_{j}\left(\begin{array}{l}
j \\
i
\end{array}\right) q_{i}(n-p)^{j-i} \\
& =\left(\sum_{i=1}^{r-3} k_{i}(n-p)^{i}\right)+m(n-p)^{r-2}+l(n-p)^{r-1}+k(n-p)^{r} \\
& +\left(\sum_{j=2}^{r-2} \sum_{i=1}^{j-1} k_{j}\left(\begin{array}{l}
j \\
i
\end{array}\right) q_{i}(n-p)^{j-i}\right)+\left(\sum_{i=1}^{r-2} l\left(\begin{array}{c}
r-1 \\
i
\end{array}\right) q_{i}(n-p)^{r-1-i}\right) \\
& +\sum_{i=1}^{r-1} k\left(\begin{array}{c}
r \\
i
\end{array}\right)(n-p)^{r-i} \\
& =m(n-p)^{r-2}+l(n-p)^{r-1}+k(n-p)^{r} \\
& +l(r-1) q(n-p)^{r-2}+k r q(n-p)^{r-1}+k\left(\begin{array}{c}
r \\
2
\end{array}\right) s(n-p)^{r-2}+\mathcal{O}\left(n^{r-3}\right) \\
& =k n^{r}-(k r(p-q)-l) n^{r-1}+(\beta(p, q, s)+m) n^{r-2}+\mathcal{O}\left(n^{r-3}\right) .
\end{aligned}
$$




\section{Comparing different groups}

In this section we use the analysis from Section 4 to prove Theorem 1.1, which collects the statements of Propositions 5.10, 5.15 and 5.16. The main technical result of the section is Proposition 5.9 which helps to break down more complex problems to problems about quotients of the form $\mathbf{S}_{n}(\mathcal{A}, H) / \mathbf{S}_{n}\left(\mathcal{A}^{\prime}, H^{\prime}\right)$, where the meaning of $\mathbf{S}_{n}(\mathcal{A}, H)$ was given by Definition 4.1. Also recall Definition 4.13 of $p(H), q(H)$ and $s(H)$ for a permutation group $H$. As usual, $r$ denotes the maximal arity, and in this section $k$ denotes the number of $r$-ary relation symbols and $l$ denotes the number of $(r-1)$-ary relation symbols.

Proposition 5.1 Suppose that $\mathcal{A}, \mathcal{A}^{\prime} \in \mathbf{S}$ are such that neither $\operatorname{Aut}(\mathcal{A})$ nor $\operatorname{Aut}\left(\mathcal{A}^{\prime}\right)$ has a fixed point. Moreover, suppose that $H$ is a subgroup of $\operatorname{Aut}(\mathcal{A})$ without fixed any point and that $H^{\prime}$ is a subgroup of $\operatorname{Aut}\left(\mathcal{A}^{\prime}\right)$ without any fixed point. Let $p=p(H)$, $q=q(H), s=s(H), p^{\prime}=p\left(H^{\prime}\right), q^{\prime}=q\left(H^{\prime}\right)$ and $s^{\prime}=s\left(H^{\prime}\right)$.

(i) The following limit exists in $\mathbb{Q} \cup\{\infty\}$ :

$$
\lim _{n \rightarrow \infty} \frac{\left|\mathbf{S}_{n}\left(\mathcal{A}^{\prime}, H^{\prime}\right)\right|}{\left|\mathbf{S}_{n}(\mathcal{A}, H)\right|} .
$$

(ii) Suppose that $r=2$.

(a) If $p-q<p^{\prime}-q^{\prime}$ or if $p-q=p^{\prime}-q^{\prime}$ and $p>p^{\prime}$, then

$$
\lim _{n \rightarrow \infty} \frac{\left|\mathbf{S}_{n}\left(\mathcal{A}^{\prime}, H^{\prime}\right)\right|}{\left|\mathbf{S}_{n}(\mathcal{A}, H)\right|}=0 .
$$

(b) If $p-q=p-q^{\prime}$ and $p=p^{\prime}$ then there is a rational number $a>0$, depending only on $\mathcal{A}, \mathcal{A}^{\prime}, H, H^{\prime}$ and the vocabulary, such that

$$
\lim _{n \rightarrow \infty} \frac{\left|\mathbf{S}_{n}\left(\mathcal{A}^{\prime}, H^{\prime}\right)\right|}{\left|\mathbf{S}_{n}(\mathcal{A}, H)\right|}=a .
$$

(iii) Suppose that $r>2$ and let $\beta(x, y, z)$ be as in Corollary 4.15. If any one of the two conditions

$$
\begin{aligned}
& p-q<p^{\prime}-q^{\prime}, \text { or } \\
& p-q=p^{\prime}-q^{\prime} \text { and } \beta(p, q, s)>\beta\left(p^{\prime}, q^{\prime}, s^{\prime}\right)
\end{aligned}
$$

hold, then

$$
\lim _{n \rightarrow \infty} \frac{\left|\mathbf{S}_{n}\left(\mathcal{A}^{\prime}, H^{\prime}\right)\right|}{\left|\mathbf{S}_{n}(\mathcal{A}, H)\right|}=0
$$


Proof (i) From Proposition 4.4 it follows that there are integers $C, C^{\prime}>0$ and polynomials $\lambda(x), \lambda^{\prime}(x)$ with integer coefficients, depending only on $\mathcal{A}, \mathcal{A}^{\prime}, H, H^{\prime}$ and the vocabulary, such that

$$
\frac{\left|\mathbf{S}_{n}\left(\mathcal{A}^{\prime}, H^{\prime}\right)\right|}{\left|\mathbf{S}_{n}(\mathcal{A}, H)\right|} \sim \frac{C^{\prime}\left(\begin{array}{c}
n \\
p^{\prime}
\end{array}\right)}{C\left(\begin{array}{l}
n \\
p
\end{array}\right)} \exp _{2}\left(\lambda^{\prime}(n)-\lambda(n)\right) .
$$

Depending on whether the leading term in the polynomial $\lambda^{\prime}(n)-\lambda(n)$ has positive degree and negative coefficient, positive degree and positive coefficient, or is constant,

$$
\exp _{2}\left(\lambda^{\prime}(n)-\lambda(n)\right)
$$

approaches $0, \infty$, or a positive real as $n \rightarrow \infty$, respectively. In the first case

$$
\left|\mathbf{S}_{n}\left(\mathcal{A}^{\prime}, H^{\prime}\right)\right| /\left|\mathbf{S}_{n}(\mathcal{A}, H)\right|
$$

approaches 0 . In the second case it approaches $\infty$. In the third case, when $\lambda^{\prime}(n)-\lambda(n)$ is constant, we get the conclusion by considering whether $p>p^{\prime}, p=p^{\prime}$ or $p<p^{\prime}$.

(ii) Suppose that $r=2$. Then Corollary 4.14 says that for some positive integers $C$ and $C^{\prime}$, depending only on $\mathcal{A}, \mathcal{A}^{\prime}, H, H^{\prime}$ and the vocabulary, we have

$$
\frac{\left|\mathbf{S}_{n}\left(\mathcal{A}^{\prime}, H^{\prime}\right)\right|}{\left|\mathbf{S}_{n}(\mathcal{A}, H)\right|} \sim \frac{C^{\prime}\left(\begin{array}{c}
n \\
p^{\prime}
\end{array}\right)}{C\left(\begin{array}{c}
n \\
p
\end{array}\right)} \exp _{2}\left(2 k\left[(p-q)-\left(p^{\prime}-q^{\prime}\right)\right] n+k\left[\left(p^{\prime}\right)^{2}-(p)^{2}\right]+l\left[p-p^{\prime}\right]\right) .
$$

From this we immediately get claims (a) and (b).

(iii) Suppose that $r>2$. Then Corollary 4.15 implies that for some positive integers $C$ and $C^{\prime}$, depending only on $\mathcal{A}, \mathcal{A}^{\prime}, H, H^{\prime}$ and the vocabulary, we have

$$
\begin{aligned}
\frac{\left|\mathbf{S}_{n}\left(\mathcal{A}^{\prime}, H^{\prime}\right)\right|}{\left|\mathbf{S}_{n}(\mathcal{A}, H)\right|} \sim \frac{C^{\prime}\left(\begin{array}{c}
n \\
p^{\prime}
\end{array}\right)}{C\left(\begin{array}{l}
n \\
p
\end{array}\right)} \exp _{2}\left(k r\left[(p-q)-\left(p^{\prime}-q^{\prime}\right)\right] n^{r-1}\right. \\
\left.\quad+\left[\beta\left(p^{\prime}, q^{\prime}, s^{\prime}\right)-\beta(p, q, s)\right] n^{r-2}+\mathcal{O}\left(n^{r-3}\right)\right) .
\end{aligned}
$$

So if $p-q<p^{\prime}-q^{\prime}$ or if $p-q=p^{\prime}-q^{\prime}$ and $\beta(p, q, s)>\beta\left(p^{\prime}, q^{\prime}, s^{\prime}\right)$, then this quotient approaches 0 as $n \rightarrow \infty$.

For the rest of this section, whenever we denote structures by $\mathcal{A}$ or $\mathcal{A}^{\prime}$, sometimes with indices, we assume that they have no fixed point. Also, whenever we denote groups by $H$ or $H^{\prime}$, sometimes with indices, we assume that they have no fixed point. Sometimes these assumptions are repeated and sometimes they are not necessary.

For different subgroups $H$ and $H^{\prime}$ of $\operatorname{Aut}(\mathcal{A})$ the sets $\mathbf{S}_{n}(\mathcal{A}, H)$ and $\mathbf{S}_{n}\left(\mathcal{A}, H^{\prime}\right)$ may have nonempty intersections, which complicates the analysis of an asymptotic estimate of the 
cardinality of a union like $\bigcup_{i=1}^{m} \mathbf{S}_{n}\left(\mathcal{A}, H_{i}\right)$. However, it turns out that for subgroups $H$ and $H^{\prime}$ of $\operatorname{Aut}(\mathcal{A})$, either $\mathbf{S}_{n}(\mathcal{A}, H)=\mathbf{S}_{n}\left(\mathcal{A}, H^{\prime}\right)$ or $\left|\mathbf{S}_{n}(\mathcal{A}, H) \cap \mathbf{S}_{n}\left(\mathcal{A}, H^{\prime}\right)\right|$ is negligibly small for large enough $n$. The results 5.3-5.8 make this statement precise.

Definition 5.2 Suppose that $\mathcal{A} \in \mathbf{S}$ and that $H$ and $H^{\prime}$ are subgroups of $\operatorname{Aut}(\mathcal{A})$. We write $H \approx_{\mathcal{A}} H^{\prime}$ if there is an automorphism $g \in \operatorname{Aut}(\mathcal{A})$ such that, for every $t=1, \ldots, r-1$, and every orbit $O$ of $H$ on $A^{t}$,

$$
g(O)=\left\{\left(g\left(a_{1}\right), \ldots, g\left(a_{t}\right)\right):\left(a_{1}, \ldots, a_{t}\right) \in O\right\}
$$

is an orbit of $H^{\prime}$ on $A^{t}$.

Observe that $\approx_{\mathcal{A}}$ is an equivalence relation on the set of subgroups of $\operatorname{Aut}(\mathcal{A})$.

Lemma 5.3 Suppose that $\mathcal{A} \in \mathbf{S}$ and that $H$ and $H^{\prime}$ are subgroups of $\operatorname{Aut}(\mathcal{A})$. If $H \approx_{\mathcal{A}} H^{\prime}$ then $\mathbf{S}_{n}(\mathcal{A}, H)=\mathbf{S}_{n}\left(\mathcal{A}, H^{\prime}\right)$.

Proof Suppose that $H \approx_{\mathcal{A}} H^{\prime}$. Recall from the discussion after the statement of Proposition 4.4 that $\mathbf{S}_{n}(\mathcal{A}, H)$ is the disjoint union of all sets of the form

$$
\mathbf{S}_{n}\left(\mathcal{A}_{X}, H\right)=\left\{\mathcal{M} \in \mathbf{S}_{n}(\mathcal{A}, H): \mathcal{M}\left\lceil\operatorname{Spt}^{*}(\mathcal{M})=\mathcal{A}_{X}\right\}\right.
$$

where $X \subseteq[n],|X|=|A|, \mathcal{A}_{X}$ has universe $X$ and $\mathcal{A}_{X} \cong \mathcal{A}$; and similarly for $H^{\prime}$. Therefore it suffices to prove that for all such $X \subseteq[n]$ and $\mathcal{A}_{X}$ we have $\mathbf{S}_{n}\left(\mathcal{A}_{X}, H\right)=\mathbf{S}_{n}\left(\mathcal{A}_{X}, H^{\prime}\right)$. By (4-4),

$$
\mathbf{S}_{n}\left(\mathcal{A}_{X}, H\right)=\bigcup_{\Pi_{1}, \ldots, \Pi_{r-1}} \mathbf{S}_{n}\left(\mathcal{A}_{X}, \Pi_{1}, \ldots, \Pi_{r-1}\right),
$$

where the union ranges over all sequences $\Pi_{1}, \ldots, \Pi_{r-1}$ of $\left(\mathcal{A}_{X}, H\right)$-partitions (see Definition 4.8) and $\mathbf{S}_{n}\left(\mathcal{A}_{X}, \Pi_{1}, \ldots, \Pi_{r-1}\right)$ was defined in (4-2). The same holds for $H^{\prime}$. Hence it suffices to show that if $\Pi_{1}, \ldots, \Pi_{r-1}$ is a sequence of $\left(\mathcal{A}_{X}, H\right)$-partitions, then $\Pi_{1}, \ldots, \Pi_{r-1}$ is a sequence of $\left(\mathcal{A}_{X}, H^{\prime}\right)$-partitions.

So suppose that $\Pi_{1}, \ldots, \Pi_{r-1}$ is a sequence of $\left(\mathcal{A}_{X}, H\right)$-partitions and hence there is an isomorphism $f: \mathcal{A} \rightarrow \mathcal{A}_{X}$ such that, for each $t=1, \ldots, r-1, \Pi_{t}$ is the set of orbits of $H_{f}=\left\{f \sigma f^{-1}: \sigma \in H\right\}$ on $X^{t}$. As we assume that $H \approx_{\mathcal{A}} H^{\prime}$, there is an automorphism $g \in \operatorname{Aut}(\mathcal{A})$ such that, for every $t=1, \ldots, r-1$ and every orbit $O$ of $H$ on $A^{t}, g(O)$ is an orbit of $H^{\prime}$ on $A^{t}$. It follows that $f^{\prime}=f g^{-1}: \mathcal{A} \rightarrow \mathcal{A}_{X}$ is an isomorphism and for each $t$ and each orbit $O^{\prime}$ of $H^{\prime}$ on $A^{t}, g^{-1}\left(O^{\prime}\right)$ is an orbit of $H$ on $A^{t}$. Consequently, for each $t, \Pi_{t}$ is the set of orbits of $H_{f^{\prime}}=\left\{f^{\prime} \sigma\left(f^{\prime}\right)^{-1}: \sigma \in H^{\prime}\right\}$ on $X^{t}$, so $\Pi_{1}, \ldots, \Pi_{r-1}$ is a sequence of $\left(\mathcal{A}_{X}, H^{\prime}\right)$-partitions. 
Lemma 5.4 Suppose that $\mathcal{A} \in \mathbf{S}$ and that $H$ and $H^{\prime}$ are subgroups of $\operatorname{Aut}(\mathcal{A})$ such that $H \not_{\mathcal{A}} H^{\prime}$. Let $X \subseteq[n],|X|=|A|$ and let $\mathcal{A}_{X}$ have universe $X$ and $\mathcal{A}_{X} \cong \mathcal{A}$. If $\Pi_{1}, \ldots, \Pi_{r-1}$ is a sequence of $\left(\mathcal{A}_{X}, H\right)$-partitions and $\Pi_{1}^{\prime}, \ldots, \Pi_{r-1}^{\prime}$ is a sequence of $\left(\mathcal{A}_{X}, H^{\prime}\right)$-partitions, then $\left(\Pi_{1}, \ldots, \Pi_{r-1}\right) \neq\left(\Pi_{1}^{\prime}, \ldots, \Pi_{r-1}^{\prime}\right)$.

Proof Suppose that $H \nsim_{\mathcal{A}} H^{\prime}, \Pi_{1}, \ldots, \Pi_{r-1}$ is a sequence of $\left(\mathcal{A}_{X}, H\right)$-partitions and $\Pi_{1}^{\prime}, \ldots, \Pi_{r-1}^{\prime}$ is a sequence of $\left(\mathcal{A}_{X}, H^{\prime}\right)$-partitions. Towards a contradiction, assume that $\left(\Pi_{1}, \ldots, \Pi_{r-1}\right)=\left(\Pi_{1}^{\prime}, \ldots, \Pi_{r-1}^{\prime}\right)$. Then there are isomorphisms $f: \mathcal{A} \rightarrow \mathcal{A}_{X}$ and $f^{\prime}: \mathcal{A} \rightarrow \mathcal{A}_{X}$ such that, for every $t=1, \ldots, r-1, \Pi_{t}$ is the set of orbits of $H_{f}=\left\{f \sigma f^{-1}: \sigma \in H\right\}$ on $X^{t}$ and $\Pi_{t}$ is also the set of orbits of $H_{f^{\prime}}=\left\{f^{\prime} \sigma\left(f^{\prime}\right)^{-1}\right.$ : $\left.\sigma \in H^{\prime}\right\}$ on $X^{t}$. So $H_{f}$ and $H_{f^{\prime}}$ have the same orbits on $X^{t}$, for each $t$. It follows that $g=\left(f^{\prime}\right)^{-1} f: \mathcal{A} \rightarrow \mathcal{A}$ is an automorphism such that for every $t=1, \ldots, r-1$ and every orbit $O$ of $H$ on $A^{t}, g(O)$ is an orbit of $H^{\prime}$ on $A^{t}$. Hence $H \approx_{\mathcal{A}} H^{\prime}$ which contradicts our assumption.

Lemma 5.5 Suppose that $\mathcal{A} \in \mathbf{S}$ and that $H$ and $H^{\prime}$ are subgroups of $\operatorname{Aut}(\mathcal{A})$ such that $H \approx_{\mathcal{A}} H^{\prime}$. Suppose that $X \subseteq[n],|X|=|A|$ and that $\mathcal{A}_{X}$ is a structure with universe $X$ such that $\mathcal{A}_{X} \cong \mathcal{A}$. If $\Pi_{1}, \ldots, \Pi_{r-1}$ is a sequence of $\left(\mathcal{A}_{X}, H\right)$-partitions and $\Pi_{1}^{\prime}, \ldots, \Pi_{r-1}^{\prime}$ is a sequence of $\left(\mathcal{A}_{X}, H^{\prime}\right)$-partitions, then

$$
\frac{\left|\mathbf{S}_{n}\left(\mathcal{A}_{X}, \Pi_{1}, \ldots, \Pi_{r-1}\right) \cap \mathbf{S}_{n}\left(\mathcal{A}_{X}, \Pi_{1}^{\prime}, \ldots, \Pi_{r-1}^{\prime}\right)\right|}{\left|\mathbf{S}_{n}\left(\mathcal{A}_{X}, \Pi_{1}, \ldots, \Pi_{r-1}\right) \cup \mathbf{S}_{n}\left(\mathcal{A}_{X}, \Pi_{1}^{\prime}, \ldots, \Pi_{r-1}^{\prime}\right)\right|} \leq \varepsilon(n),
$$

where $\varepsilon(n) \rightarrow 0$ as $n \rightarrow \infty$ and the function $\varepsilon: \mathbb{N} \rightarrow \mathbb{R}$ only depends on $\mathcal{A}, H, H^{\prime}$ and the vocabulary.

Proof The assumptions and Lemma 5.4 imply that $\left(\Pi_{1}, \ldots, \Pi_{r-1}\right) \neq\left(\Pi_{1}^{\prime}, \ldots, \Pi_{r-1}^{\prime}\right)$. Lemma 4.11 is applicable to each one of the sequences $\Pi_{1}, \ldots, \Pi_{r-1}$ and $\Pi_{1}^{\prime}, \ldots, \Pi_{r-1}^{\prime}$. Now observe that the proof of Lemma 4.12 works out in exactly the same way even if $\Pi_{1}, \ldots, \Pi_{r-1}$ is a sequence of $\left(\mathcal{A}_{X}, H\right)$-partitions and $\Pi_{1}^{\prime}, \ldots, \Pi_{r-1}^{\prime}$ is a sequence of $\left(\mathcal{A}_{X}, H^{\prime}\right)$-partitions; the proof of Lemma 4.12 only uses the assumption that the sequences $\Pi_{1}, \ldots, \Pi_{r-1}$ and $\Pi_{1}^{\prime}, \ldots, \Pi_{r-1}^{\prime}$ are different. Hence Lemma 5.5 is proved.

Remark 5.6 If $\mathcal{A} \in \mathbf{S}$ and $H \subseteq \operatorname{Aut}(\mathcal{A})$ is a subgroup, then, by Lemma 4.6 and the argument between Proposition 4.4 and Lemma 4.6,

$$
\left|\mathbf{S}_{n}(\mathcal{A}, H)\right| \sim C\left(\begin{array}{c}
n \\
|A|
\end{array}\right)\left|\mathbf{S}_{n}\left(\mathcal{A}_{X}, H\right)\right|
$$

where $C$ is a constant that depends only on $\mathcal{A}, H$ and the vocabulary, $X \subseteq[n], \mathcal{A}_{X}$ is a structure with universe $X$ such that $\mathcal{A}_{X} \cong \mathcal{A}$ (and $\mathbf{S}_{n}\left(\mathcal{A}_{X}, H\right)$ is as defined in (4-1)). 
Corollary 5.7 Suppose that $\mathcal{A} \in \mathbf{S}$ and that $H$ and $H^{\prime}$ are subgroups of $\operatorname{Aut}(\mathcal{A})$ such that $H \nsim_{\mathcal{A}} H^{\prime}$. Then

$$
\frac{\left|\mathbf{S}_{n}(\mathcal{A}, H) \cap \mathbf{S}_{n}\left(\mathcal{A}, H^{\prime}\right)\right|}{\left|\mathbf{S}_{n}(\mathcal{A}, H) \cup \mathbf{S}_{n}\left(\mathcal{A}, H^{\prime}\right)\right|} \leq \varepsilon(n),
$$

where $\varepsilon(n) \rightarrow 0$ as $n \rightarrow \infty$ and the function $\varepsilon$ only depends on $\mathcal{A}, H, H^{\prime}$ and the vocabulary.

Proof Suppose that $\mathcal{A} \in \mathbf{S}$ and that $H$ and $H^{\prime}$ are subgroups of $\operatorname{Aut}(\mathcal{A})$ such that $H \nsim_{\mathcal{A}} H^{\prime}$. By Remark 5.6, it suffices to prove that there is a function $\varepsilon(n)$, depending only on $\mathcal{A}, H$ and the vocabulary, such that $\lim _{n \rightarrow \infty} \varepsilon(n)=0$ and for every $X \subseteq[n]$ and $\mathcal{A}_{X}$ as above,

$$
\frac{\left|\mathbf{S}_{n}\left(\mathcal{A}_{X}, H\right) \cap \mathbf{S}_{n}\left(\mathcal{A}_{X}, H^{\prime}\right)\right|}{\left|\mathbf{S}_{n}\left(\mathcal{A}_{X}, H\right) \cup \mathbf{S}_{n}\left(\mathcal{A}_{X}, H^{\prime}\right)\right|} \leq \varepsilon(n) .
$$

Recall from (4-4) that

$$
\mathbf{S}_{n}\left(\mathcal{A}_{X}, H\right)=\bigcup_{\Pi_{1}, \ldots, \Pi_{r-1}} \mathbf{S}_{n}\left(\mathcal{A}_{X}, \Pi_{1}, \ldots, \Pi_{r-1}\right)
$$

where the union ranges over all sequences of $\left(\mathcal{A}_{X}, H\right)$-partitions. Given $X$ and $\mathcal{A}_{X}$ there is a finite bound $\alpha$, depending only on $\mathcal{A}, H, H^{\prime}$ and the vocabulary, such that there are at most $\alpha$ sequences $\Pi_{1}, \ldots, \Pi_{r-1}$ of $\left(\mathcal{A}_{X}, H\right)$-partitions and at most $\alpha$ sequences $\Pi_{1}^{\prime}, \ldots, \Pi_{r-1}^{\prime}$ of $\left(\mathcal{A}_{X}, H^{\prime}\right)$-partitions. Therefore the bound we are looking for is a fixed multiple of the bound given by Lemma 5.5.

Lemma 5.8 Suppose that $\mathcal{A} \in \mathbf{S}$ and that $H_{i}, i=1, \ldots, m$, are subgroups of $\operatorname{Aut}(\mathcal{A})$ such that if $i \neq j$, then $H_{i} \not \sim_{\mathcal{A}} H_{j}$ and $\left|\mathbf{S}_{n}\left(\mathcal{A}, H_{i}\right)\right| /\left|\mathbf{S}_{n}\left(\mathcal{A}, H_{j}\right)\right|$ converges to a positive rational number. Then

$$
\left|\bigcup_{i=1}^{m} \mathbf{S}_{n}\left(\mathcal{A}, H_{i}\right)\right| \sim \sum_{i=1}^{m}\left|\mathbf{S}_{n}\left(\mathcal{A}, H_{i}\right)\right| .
$$

Proof From Corollary 5.7 it follows that if $i \neq i^{\prime}$ then

$$
\left|\mathbf{S}_{n}\left(\mathcal{A}, H_{i}\right) \cap \mathbf{S}_{n}\left(\mathcal{A}, H_{i^{\prime}}\right)\right| \leq o(1)\left(\left|\mathbf{S}_{n}\left(\mathcal{A}, H_{i}\right)\right|+\left|\mathbf{S}_{n}\left(\mathcal{A}, H_{i^{\prime}}\right)\right|\right),
$$

where the bound $o(1)$ depends only on $\mathcal{A}, H_{1}, \ldots, H_{m}$ and the vocabulary. Now the assumption that $\left|\mathbf{S}_{n}\left(\mathcal{A}, H_{i}\right)\right| /\left|\mathbf{S}_{n}\left(\mathcal{A}, H_{i^{\prime}}\right)\right|$ converges to a positive rational number and (5-1) implies that if $i \neq i^{\prime}$, then

$$
\left|\mathbf{S}_{n}\left(\mathcal{A}, H_{i}\right) \cap \mathbf{S}_{n}\left(\mathcal{A}, H_{i^{\prime}}\right)\right| \leq o(1)\left|\mathbf{S}_{n}\left(\mathcal{A}, H_{i}\right)\right|,
$$


for some bound $o(1)$ which depends only on $\mathcal{A}, H_{1}, \ldots, H_{m}$ and the vocabulary. If we let $\mathbf{U}_{n}$ be the union of all intersections

$$
\mathbf{S}_{n}\left(\mathcal{A}, H_{i}\right) \cap \mathbf{S}_{n}\left(\mathcal{A}, H_{i^{\prime}}\right)
$$

where $\left\{i, i^{\prime}\right\}$ range over all subsets of $[m]$ with cardinality 2 , then we get, for every $i$,

$$
\left|\mathbf{S}_{n}\left(\mathcal{A}, H_{i}\right)\right|-\left|\mathbf{U}_{n}\right| \geq(1-o(1))\left|\mathbf{S}_{n}\left(\mathcal{A}, H_{i}\right)\right|,
$$

where the bound $o(1)$ depends only on $\mathcal{A}$ and $H_{1}, \ldots, H_{m}$. Now we get

$$
\left|\bigcup_{i=1}^{m} \mathbf{S}_{n}\left(\mathcal{A}, H_{i}\right)\right| \geq \sum_{i=1}^{m}\left(\left|\mathbf{S}_{n}\left(\mathcal{A}, H_{i}\right)\right|-\left|\mathbf{U}_{n}\right|\right) \geq(1-o(1)) \sum_{i=1}^{m}\left|\mathbf{S}_{n}\left(\mathcal{A}, H_{i}\right)\right| .
$$

Since also

$$
\left|\bigcup_{i=1}^{m} \mathbf{S}_{n}\left(\mathcal{A}, H_{i}\right)\right| \leq \sum_{i=1}^{m}\left|\mathbf{S}_{n}\left(\mathcal{A}, H_{i}\right)\right|
$$

the proof of the lemma is finished.

Proposition 5.9 Let $\mathcal{A}_{1}, \ldots, \mathcal{A}_{m}, \mathcal{A}_{1}^{\prime}, \ldots, \mathcal{A}_{m^{\prime}}^{\prime} \in \mathbf{S}$ be such that none of them has any fixed point. Suppose that for every $i=1, \ldots, m$ and $j=1, \ldots, l_{i}, H_{i, j}$ is a subgroup of $\operatorname{Aut}\left(\mathcal{A}_{i}\right)$ without any fixed point and that for every $i=1, \ldots, m^{\prime}$ and $j=1, \ldots, l_{i}^{\prime} H_{i, j}^{\prime}$ is a subgroup of $\operatorname{Aut}\left(\mathcal{A}_{i}^{\prime}\right)$ without any fixed point. Then the following limit exists in $\mathbb{Q} \cup\{\infty\}:$

$$
\lim _{n \rightarrow \infty} \frac{\left|\bigcup_{i=1}^{m^{\prime}} \bigcup_{j=1}^{l_{i}^{\prime}} \mathbf{S}_{n}\left(\mathcal{A}_{i}^{\prime}, H_{i, j}^{\prime}\right)\right|}{\left|\bigcup_{i=1}^{m} \bigcup_{j=1}^{l_{i}} \mathbf{S}_{n}\left(\mathcal{A}_{i}, H_{i, j}\right)\right|}
$$

Proof By just removing if necessary some $\mathcal{A}_{i}$ or $\mathcal{A}_{i}^{\prime}$ and relabelling the rest of the structures and groups, we may assume that $\mathcal{A}_{i} \neq \mathcal{A}_{j}$ if $i \neq j$ and $\mathcal{A}_{i}^{\prime} \neq \mathcal{A}_{j}^{\prime}$ if $i \neq j$. Also, by Lemma 5.3, we may assume that $H_{i, j} \not \mathcal{A}_{\mathcal{A}_{i}} H_{i, j^{\prime}}$ if $j \neq j^{\prime}$ and that $H_{i, j}^{\prime} \not_{\mathcal{A}_{i}^{\prime}} H_{i, j^{\prime}}^{\prime}$ if $j \neq j^{\prime}$.

By Proposition 5.1 (i), for all $1 \leq i \leq m$ and all $1 \leq j, j^{\prime} \leq l_{i}$,

$$
\left|\mathbf{S}_{n}\left(\mathcal{A}_{i}, H_{i, j^{\prime}}\right)\right| /\left|\mathbf{S}_{n}\left(\mathcal{A}_{i}, H_{i, j}\right)\right|
$$

converges to a rational number or approaches infinity, as $n \rightarrow \infty$. The same holds for all $1 \leq i \leq m^{\prime}$, all $1 \leq j, j^{\prime} \leq l_{i}^{\prime}$ and $\left|\mathbf{S}_{n}\left(\mathcal{A}_{i}^{\prime}, H_{i, j^{\prime}}^{\prime}\right)\right| /\left|\mathbf{S}_{n}\left(\mathcal{A}_{i}^{\prime}, H_{i, j}^{\prime}\right)\right|$. Therefore it suffices to prove (5-2) under the assumption that for all $1 \leq i \leq m$ and all $1 \leq j, j^{\prime} \leq l_{i},\left|\mathbf{S}_{n}\left(\mathcal{A}_{i}, H_{i, j^{\prime}}\right)\right| /\left|\mathbf{S}_{n}\left(\mathcal{A}_{i}, H_{i, j}\right)\right|$ converges to a positive rational number and 
for all $1 \leq i \leq m^{\prime}$ and all $1 \leq j, j^{\prime} \leq l_{i}^{\prime},\left|\mathbf{S}_{n}\left(\mathcal{A}_{i}^{\prime}, H_{i, j^{\prime}}^{\prime}\right)\right| /\left|\mathbf{S}_{n}\left(\mathcal{A}_{i}^{\prime}, H_{i, j}^{\prime}\right)\right|$ converges to a positive rational number.

From our assumptions we have $\mathbf{S}_{n}\left(\mathcal{A}_{i}, H_{i, j}\right) \cap \mathbf{S}_{n}\left(\mathcal{A}_{i^{\prime}}, H_{i^{\prime}, j^{\prime}}\right)=\emptyset$ if $i \neq i^{\prime}$ (and the same for $\mathcal{A}_{i}^{\prime}, \mathcal{A}_{j}^{\prime}, H_{i, j}^{\prime}$ and $H_{i, j^{\prime}}^{\prime}$ ). By applying Lemma 5.8 and the assumptions, we now get

$$
\begin{aligned}
& \frac{\left|\bigcup_{i=1}^{m^{\prime}} \bigcup_{j=1}^{l_{i}^{\prime}} \mathbf{S}_{n}\left(\mathcal{A}_{i}^{\prime}, H_{i, j}^{\prime}\right)\right|}{\left|\bigcup_{i=1}^{m} \bigcup_{j=1}^{l_{i}} \mathbf{S}_{n}\left(\mathcal{A}_{i}, H_{i, j}\right)\right|}=\frac{\sum_{i=1}^{m^{\prime}} \sum_{j=1}^{l_{i}^{\prime}}\left|\mathbf{S}_{n}\left(\mathcal{A}_{i}^{\prime}, H_{i, j}^{\prime}\right)\right|}{\sum_{i=1}^{m} \sum_{j=1}^{l_{i}}\left|\mathbf{S}_{n}\left(\mathcal{A}_{i}, H_{i, j}\right)\right|} \\
= & \frac{\left|\mathbf{S}_{n}\left(\mathcal{A}_{1}^{\prime}, H_{1,1}\right)\right|}{\sum_{i=1}^{m} \sum_{j=1}^{l_{i}}\left|\mathbf{S}_{n}\left(\mathcal{A}_{i}\right)\right|}+\ldots+\frac{\left|\mathbf{S}_{n}\left(\mathcal{A}_{m^{\prime}}^{\prime}, H_{m^{\prime}} l_{m^{\prime}}\right)\right|}{\sum_{i=1}^{m} \sum_{j=1}^{l_{i}}\left|\mathbf{S}_{n}\left(\mathcal{A}_{i}, H_{i, j}\right)\right|} \\
= & \left(\sum_{i=1}^{m} \sum_{j=1}^{l_{i}} \frac{\left|\mathbf{S}_{n}\left(\mathcal{A}_{i}, H_{i, j}\right)\right|}{\left|\mathbf{S}_{n}\left(A_{1}^{\prime}, H_{1,1}^{\prime}\right)\right|}\right)^{-1}+\ldots+\left(\sum_{i=1}^{m} \sum_{j=1}^{l_{i}} \frac{\left|\mathbf{S}_{n}\left(\mathcal{A}_{i}, H_{i, j}\right)\right|}{\mid \mathbf{S}_{n}\left(A_{m^{\prime}}^{\prime}, H_{m^{\prime}, l_{m^{\prime}}}^{\prime}\right)}\right)^{-1} .
\end{aligned}
$$

Note that Proposition 4.4 implies that, for all $i, j$ and all sufficiently large $n$, $\left|\mathbf{S}_{n}\left(\mathcal{A}_{i}, H_{i, j}\right)\right|>0$, and similarly for and $\mathcal{A}_{i}^{\prime}$ and $H_{i, j}^{\prime}$, so we do not divide by zero in the above expression if $n$ is large enough. By Proposition 5.1 (i), for every choice of $i, i^{\prime}, j$ and $j^{\prime},\left|\mathbf{S}_{n}\left(\mathcal{A}_{i}, H_{i, j}\right)\right| /\left|\mathbf{S}_{n}\left(\mathcal{A}_{i^{\prime}}^{\prime}, H_{i^{\prime}, j^{\prime}}^{\prime}\right)\right|$ converges to a rational number or approaches $\infty$. This implies (5-2) so the proposition is proved.

Proposition 5.10 Let $G$ and $G^{\prime}$ be finite groups. Then the following limit exists in $\mathbb{Q} \cup\{\infty\}:$

$$
\lim _{n \rightarrow \infty} \frac{\left|\left\{\mathcal{M} \in \mathbf{S}_{n}: G^{\prime} \leq \operatorname{Aut}(\mathcal{M})\right\}\right|}{\left|\left\{\mathcal{M} \in \mathbf{S}_{n}: G \leq \operatorname{Aut}(\mathcal{M})\right\}\right|} .
$$

Proof Let $G$ and $G^{\prime}$ be finite groups. Lemma 4.3 implies that there are finitely many

$$
\begin{aligned}
& \text { structures } \mathcal{A}_{1}, \ldots, \mathcal{A}_{m}, \mathcal{A}_{1}^{\prime}, \ldots, \mathcal{A}_{m^{\prime}}^{\prime} \in \mathbf{S}, \\
& \text { subgroups } H_{i, 1}, \ldots, H_{i, l_{i}} \subseteq \operatorname{Aut}\left(\mathcal{A}_{i}\right), \text { for } i=1, \ldots, m, \text { and } \\
& \text { subgroups } H_{i, 1}^{\prime}, \ldots, H_{i, l_{i}^{\prime}}^{\prime} \subseteq \operatorname{Aut}\left(\mathcal{A}_{i}^{\prime}\right), \text { for } i=1, \ldots, m^{\prime},
\end{aligned}
$$

such that the following hold:

(i) None of the permutation groups $\operatorname{Aut}\left(\mathcal{A}_{i}\right), \operatorname{Aut}\left(\mathcal{A}_{i}^{\prime}\right), H_{i, j}$ or $H_{i, j}$ has any fixed point.

(ii) $\left|\left\{\mathcal{M} \in \mathbf{S}_{n}: G \leq \operatorname{Aut}(\mathcal{M})\right\}\right| \sim\left|\bigcup_{i=1}^{m} \bigcup_{j=1}^{l_{i}} \mathbf{S}_{n}\left(\mathcal{A}_{i}, H_{i, j}\right)\right|$ as $n \rightarrow \infty$.

(iii) $\left|\left\{\mathcal{M} \in \mathbf{S}_{n}: G^{\prime} \leq \operatorname{Aut}(\mathcal{M})\right\}\right| \sim\left|\bigcup_{i=1}^{m^{\prime}} \bigcup_{j=1}^{l_{i}^{\prime}} \mathbf{S}_{n}\left(\mathcal{A}_{i}^{\prime}, H_{i, j}^{\prime}\right)\right|$ as $n \rightarrow \infty$. 
Hence it suffices to prove that

$$
\lim _{n \rightarrow \infty} \frac{\left|\bigcup_{i=1}^{m^{\prime}} \bigcup_{j=1}^{l_{i}^{\prime}} \mathbf{S}_{n}\left(\mathcal{A}_{i}^{\prime}, H_{i, j}^{\prime}\right)\right|}{\left|\bigcup_{i=1}^{m} \bigcup_{j=1}^{l_{i}} \mathbf{S}_{n}\left(\mathcal{A}_{i}, H_{i, j}\right)\right|}
$$

exists in $\mathbb{Q} \cup\{\infty\}$. But this follows immediately from Proposition 5.9.

By the definition of $\mathbf{S}_{n}(\mathcal{A}, H)$ (Definition 4.1), for every $\mathcal{M} \in \mathbf{S}_{n}(\mathcal{A}, H)$, $\operatorname{Aut}(\mathcal{M}) \mid$ $\operatorname{Spt}^{*}(\mathcal{M})$ has a subgroup $H_{f}$ such that $H_{f} \cong_{P} H$. The next lemma shows that for almost all $\mathcal{M} \in \mathbf{S}(\mathcal{A}, H)$ any such $H_{f}$ has the same orbits as $\operatorname{Aut}(\mathcal{M})\left\lceil\operatorname{Spt}^{*}(\mathcal{M})\right.$.

Lemma 5.11 Suppose that $\mathcal{A} \in \mathbf{S}$ has no fixed point and that $H$ is a subgroup of $\operatorname{Aut}(\mathcal{A})$ without any fixed point. There is a function $\varepsilon(n)$, depending only on $\mathcal{A}, H$ and the vocabulary, such that $\lim _{n \rightarrow \infty} \varepsilon(n)=0$ and the proportion of $\mathcal{M} \in \mathbf{S}_{n}(\mathcal{A}, H)$ with the following property is at most $\varepsilon(n)$ :

(*) For some isomorphism $f: \mathcal{A} \rightarrow \mathcal{M}\left\lceil\operatorname{Spt}^{*}(\mathcal{M})\right.$ such that $H_{f}=\left\{f \sigma f^{-1}: \sigma \in H\right\}$ is a subgroup of $\operatorname{Aut}(\mathcal{M})\left\lceil\operatorname{Spt}^{*}(\mathcal{M})\right.$, there is $t \in\{1, \ldots, r-1\}$ such that the orbits of $\operatorname{Aut}(\mathcal{M}) \mid \operatorname{Spt}^{*}(\mathcal{M})$ on $\operatorname{Spt}^{*}(\mathcal{M})^{t}$ are not the same as the orbits of $H_{f}$ on $\operatorname{Spt}^{*}(\mathcal{M})^{t}$.

Proof Let $\mathcal{M} \in \mathbf{S}_{n}(\mathcal{A}, H), X=\operatorname{Spt}^{*}(\mathcal{M})$ and $\mathcal{A}_{X}=\mathcal{M} \uparrow X$, so $\mathcal{A}_{X} \cong \mathcal{A}$. Moreover, let $f: \mathcal{A} \rightarrow \mathcal{A}_{X}$ be an isomorphism and assume that $H_{f}=\left\{f \sigma f^{-1}: \sigma \in H\right\}$ is a subgroup of $\operatorname{Aut}(\mathcal{M}) \mid X$. Suppose that for some $t \in\{1, \ldots, r-1\}$ the orbits of $\operatorname{Aut}(\mathcal{M}) \mid X$ on $X^{t}$ are not the same as the orbits of $H_{f}$ on $X^{t}$. It follows that $\operatorname{Aut}(\mathcal{M}) \mid X$ has fewer orbits on $X^{t}$ than $H_{f}$. Hence there is a subgroup $H^{\prime}$ of $\operatorname{Aut}(\mathcal{A})$ such that $H \subseteq H^{\prime}, H^{\prime}$ has fewer orbits than $H$ on $A^{t}$ and $\mathcal{M} \in \mathbf{S}_{n}\left(\mathcal{A}, H^{\prime}\right)$. It follows that $H^{\prime} \approx_{\mathcal{A}} H$ and that

$$
\mathcal{M} \in \mathbf{S}_{n}\left(\mathcal{A}, H^{\prime}\right) \cap \mathbf{S}_{n}(\mathcal{A}, H) .
$$

Now Corollary 5.7 implies that

$$
\left|\mathbf{S}_{n}(\mathcal{A}, H) \cap \mathbf{S}_{n}\left(\mathcal{A}, H^{\prime}\right)\right| \leq \varepsilon(n)\left|\mathbf{S}_{n}(\mathcal{A}, H) \cup \mathbf{S}_{n}\left(\mathcal{A}, H^{\prime}\right)\right|,
$$

where $\varepsilon(n) \rightarrow 0$ as $n \rightarrow \infty$ and $\varepsilon(n)$ only depends on $\mathcal{A}, H, H^{\prime}$ and the vocabulary. Since $H$ is a subgroup of $H^{\prime}$ we have

$$
\mathbf{S}_{n}\left(\mathcal{A}, H^{\prime}\right) \subseteq \mathbf{S}_{n}(\mathcal{A}, H)
$$

which implies that

$$
\left|\mathbf{S}_{n}\left(\mathcal{A}, H^{\prime}\right)\right| \leq \varepsilon(n)\left|\mathbf{S}_{n}(\mathcal{A}, H)\right|
$$


We have proved that if $\mathcal{M} \in \mathbf{S}_{n}(\mathcal{A}, H)$ and satisfies $(*)$ then $\mathcal{M} \in \mathbf{S}_{n}\left(\mathcal{A}, H^{\prime}\right)$ for some subgroup $H^{\prime}$ of $\operatorname{Aut}(\mathcal{A})$ such that (5-3) holds. As the number of subgroups $H^{\prime}$ of $\operatorname{Aut}(\mathcal{A})$ is finite and depends only on $\mathcal{A}$ the lemma follows.

Definition 5.12 Suppose that $\mathcal{A} \in \mathbf{S}$ has no fixed point and that $H$ is a subgroup of $\operatorname{Aut}(\mathcal{A})$ without any fixed point. For $\mathcal{M} \in \mathbf{S}_{n}(\mathcal{A}, H)$ we say that $H$ is the full automorphism group of $\mathcal{M}$ if for every isomorphism $f: \mathcal{A} \rightarrow \mathcal{M}\left\lceil\operatorname{Spt}^{*}(\mathcal{M})\right.$ such that $H_{f}=\left\{f \sigma f^{-1}: \sigma \in H\right\}$ is a subgroup of $\operatorname{Aut}(\mathcal{M})\left\lceil\operatorname{Spt}^{*}(\mathcal{M})\right.$ we have $H_{f}=\operatorname{Aut}(\mathcal{M})\left\lceil\operatorname{Spt}^{*}(\mathcal{M})\right.$.

Lemma 5.13 Suppose that $\mathcal{A} \in \mathbf{S}$ has no fixed point and that $H$ is a subgroup of $\operatorname{Aut}(\mathcal{A})$ without any fixed point. The proportion of $\mathcal{M} \in \mathbf{S}_{n}(\mathcal{A}, H)$ such that $H$ is the full automorphism group of $\mathcal{M}$ converges to either 0 or 1 as $n \rightarrow \infty$.

Proof By Lemma 5.11, it suffices to consider $\mathcal{M} \in \mathbf{S}_{n}(\mathcal{A}, H)$ with the following property:

For every isomorphism $f: \mathcal{A} \rightarrow \mathcal{M}\left\lceil\operatorname{Spt}^{*}(\mathcal{M})\right.$ such that $H_{f}$ is a subgroup of $\operatorname{Aut}(\mathcal{M})\left\lceil\operatorname{Spt}^{*}(\mathcal{M}), H_{f}\right.$ and $\operatorname{Aut}(\mathcal{M})\left\lceil\operatorname{Spt}^{*}(\mathcal{M})\right.$ have the same orbits on $\operatorname{Spt}^{*}(\mathcal{M})^{t}$ for all $t=1, \ldots, r-1$.

For such $\mathcal{M}$ the question whether there is $g \in \operatorname{Aut}(\mathcal{M})\left\lceil\operatorname{Spt}^{*}(\mathcal{M})\right.$ such that $g \notin H_{f}$ depends only on the isomorphism type of $\mathcal{A}, H$ and the isomorphism $f: \mathcal{A} \rightarrow \mathcal{M}$ 个 $\operatorname{Spt}^{*}(\mathcal{M})$. In fact, it depends only on the isomorphism type of $\mathcal{A}$ and $H$. For if $f$ and $f^{\prime}$ are isomorphisms from $\mathcal{A}$ to $\mathcal{M}\left\lceil\operatorname{Spt}^{*}(\mathcal{M}), H_{f}=\operatorname{Aut}(\mathcal{M})\left\lceil\operatorname{Spt}^{*}(\mathcal{M})\right.\right.$ and $g \in \operatorname{Aut}(\mathcal{M})\left\lceil\operatorname{Spt}^{*}(\mathcal{M})\right.$ does not belong to $H_{f^{\prime}}$, then, since $\left|H_{f}\right|=\left|H_{f^{\prime}}\right|$ (because $f^{\prime} f^{-1}$ is an isomorphism from $H_{f}$ to $H_{f^{\prime}}$ as permutation groups), we get $\left|H_{f^{\prime}}\right|<|\operatorname{Aut}(\mathcal{M})|=$ $\left|H_{f}\right|=\left|H_{f^{\prime}}\right|$, which is impossible.

Lemma 5.14 Suppose that $\mathcal{A} \in \mathbf{S}$ has no fixed point and that $H \subseteq \operatorname{Aut}(\mathcal{A})$ is a subgroup without any fixed point. For every group $G \leq H$, the proportion of $\mathcal{M} \in \mathbf{S}_{n}(\mathcal{A}, H)$ such that $G \cong \operatorname{Aut}(\mathcal{M})$ converges to either 0 or 1 as $n \rightarrow \infty$.

Proof Suppose that $\mathcal{A} \in \mathbf{S}$ has no fixed point, that $H \subseteq \operatorname{Aut}(\mathcal{A})$ is a subgroup without any fixed point and $G \leq H$. Since $\operatorname{Aut}(\mathcal{M}) \cong \operatorname{Aut}(\mathcal{M})\left\lceil\operatorname{Spt}^{*}(\mathcal{M})\right.$ for every $\mathcal{M} \in \mathbf{S}$, Lemma 5.13 implies that the proportion of $\mathcal{M} \in \mathbf{S}_{n}(\mathcal{A}, H)$ such that $H \cong \operatorname{Aut}(\mathcal{M})$ converges to either 0 or 1 as $n \rightarrow \infty$. If $G \cong H$ it follows that the proportion of $\mathcal{M} \in \mathbf{S}_{n}(\mathcal{A}, H)$ such that $G \cong \operatorname{Aut}(\mathcal{M})$ converges to either 0 or 1 as $n \rightarrow \infty$. If $G$ is isomorphic to a proper subgroup of $H$ then, since $H \leq \operatorname{Aut}(\mathcal{M})$ for every $\mathcal{M} \in \mathbf{S}_{n}(\mathcal{A}, H)$, it follows that $G \neq \operatorname{Aut}(\mathcal{M})$ for every $\mathcal{M} \in \mathbf{S}_{n}(\mathcal{A}, H)$. 
Proposition 5.15 If $G$ is a finite group then there is a rational number $0 \leq a \leq 1$ such that

$$
\lim _{n \rightarrow \infty} \frac{\left|\left\{\mathcal{M} \in \mathbf{S}_{n}: G \cong \operatorname{Aut}(\mathcal{M})\right\}\right|}{\left|\left\{\mathcal{M} \in \mathbf{S}_{n}: G \leq \operatorname{Aut}(\mathcal{M})\right\}\right|}=a
$$

Proof Let $G$ be a finite group. By Lemma 4.3, there are finitely many

$$
\begin{aligned}
& \text { structures } \mathcal{A}_{1}, \ldots, \mathcal{A}_{m} \in \mathbf{S} \text { and } \\
& \text { subgroups } H_{i, 1}, \ldots, H_{i, l_{i}} \subseteq \operatorname{Aut}\left(\mathcal{A}_{i}\right), \text { for } i=1, \ldots, m
\end{aligned}
$$

such that:

(i) None of the permutation groups $\operatorname{Aut}\left(\mathcal{A}_{i}\right)$ or $H_{i, j}$ has a fixed point.

(ii) $G \leq H_{i, j}$ for all $i$ and $j$.

(iii) $\left|\left\{\mathcal{M} \in \mathbf{S}_{n}: G \leq \operatorname{Aut}(\mathcal{M})\right\}\right| \sim\left|\bigcup_{i=1}^{m} \bigcup_{j=1}^{l_{i}} \mathbf{S}_{n}\left(\mathcal{A}_{i}, H_{i, j}\right)\right|$ as $n \rightarrow \infty$.

Lemma 5.14 says that for every $\mathcal{A}_{i}$ and every $H_{i, j}$ the proportion of $\mathcal{M} \in \mathbf{S}_{n}\left(\mathcal{A}_{i}, H_{i, j}\right)$ such that $G \cong \operatorname{Aut}(\mathcal{M})$ converges to either 0 or 1 . Let $\left(\mathcal{A}_{i}^{\prime}, H_{i, j}^{\prime}\right), i=1, \ldots, m^{\prime}$, $j=1, \ldots, l_{i}^{\prime}$, enumerate the pairs $\left(\mathcal{A}_{i}, H_{i, j}\right)$ such that the proportion of $\mathcal{M} \in \mathbf{S}_{n}\left(\mathcal{A}_{i}, H_{i, j}\right)$ for which $G \cong \operatorname{Aut}(\mathcal{M})$ converges to 1 . Then

$$
\frac{\left|\left\{\mathcal{M} \in \mathbf{S}_{n}: G \cong \operatorname{Aut}(\mathcal{M})\right\}\right|}{\left|\left\{\mathcal{M} \in \mathbf{S}_{n}: G \leq \operatorname{Aut}(\mathcal{M})\right\}\right|} \sim \frac{\left|\bigcup_{i=1}^{m^{\prime}} \bigcup_{j=1}^{l_{i}^{\prime}} \mathbf{S}_{n}\left(\mathcal{A}_{i}^{\prime}, H_{i, j}^{\prime}\right)\right|}{\left|\bigcup_{i=1}^{m} \bigcup_{j=1}^{l_{i}} \mathbf{S}_{n}\left(\mathcal{A}_{i}, H_{i, j}\right)\right|} \rightarrow a \text { as } n \rightarrow \infty
$$

for some rational $0 \leq a \leq 1$, by Proposition 5.9.

Proposition 5.16 Let $G$ and $G^{\prime}$ be finite groups. Then the following limit exists in $\mathbb{Q} \cup\{\infty\}:$

$$
\lim _{n \rightarrow \infty} \frac{\left|\left\{\mathcal{M} \in \mathbf{S}_{n}: G^{\prime} \cong \operatorname{Aut}(\mathcal{M})\right\}\right|}{\left|\left\{\mathcal{M} \in \mathbf{S}_{n}: G \cong \operatorname{Aut}(\mathcal{M})\right\}\right|}
$$

Proof By Lemma 4.3, there are finitely many

$$
\begin{aligned}
& \text { structures } \mathcal{A}_{1}, \ldots, \mathcal{A}_{m}, \mathcal{A}_{1}^{\prime}, \ldots, \mathcal{A}_{m^{\prime}}^{\prime} \in \mathbf{S}, \\
& \text { subgroups } H_{i, 1}, \ldots, H_{i, l_{i}} \subseteq \operatorname{Aut}\left(\mathcal{A}_{i}\right), \text { for } i=1, \ldots, m, \text { and } \\
& \text { subgroups } H_{i, 1}^{\prime}, \ldots, H_{i, l_{i}^{\prime}}^{\prime} \subseteq \operatorname{Aut}\left(\mathcal{A}_{i}^{\prime}\right), \text { for } i=1, \ldots, m^{\prime},
\end{aligned}
$$

such that:

(i) None of the permutation groups $\operatorname{Aut}\left(\mathcal{A}_{i}\right), \operatorname{Aut}\left(\mathcal{A}_{i}^{\prime}\right), H_{i, j}$ or $H_{i, j}$ has any fixed point. 
(ii) $G \leq H_{i, j}$ and $G^{\prime} \leq H_{i, j}^{\prime}$ for all $i, j$.

(iii) $\left|\left\{\mathcal{M} \in \mathbf{S}_{n}: G \leq \operatorname{Aut}(\mathcal{M})\right\}\right| \sim\left|\bigcup_{i=1}^{m} \bigcup_{j=1}^{l_{i}} \mathbf{S}_{n}\left(\mathcal{A}_{i}, H_{i, j}\right)\right|$ as $n \rightarrow \infty$.

(iv) $\left|\left\{\mathcal{M} \in \mathbf{S}_{n}: G^{\prime} \leq \operatorname{Aut}(\mathcal{M})\right\}\right| \sim\left|\bigcup_{i=1}^{m^{\prime}} \bigcup_{j=1}^{l_{i}^{\prime}} \mathbf{S}_{n}\left(\mathcal{A}_{i}^{\prime}, H_{i, j}^{\prime}\right)\right|$ as $n \rightarrow \infty$.

As in the proof of Proposition 5.15 we now use Lemma 5.14. So let $\left(\mathcal{A}_{i}^{*}, H_{i, j}^{*}\right)$, $i=1, \ldots, m^{*}, j=1, \ldots, l_{i}^{*}$, enumerate all pairs $\left(\mathcal{A}_{i}, H_{i, j}\right)$ such that the proportion of $\mathcal{M} \in \mathbf{S}_{n}\left(\mathcal{A}_{i}, H_{i, j}\right)$ for which $G \cong \operatorname{Aut}(\mathcal{M})$ converges to 1 . Similarly, let $\left(\mathcal{A}_{i}^{+}, H_{i, j}^{+}\right)$, $i=1, \ldots, m^{+}, j=1, \ldots, l_{i}^{+}$, enumerate all pairs $\left(\mathcal{A}_{i}^{\prime}, H_{i, j}^{\prime}\right)$ such that the proportion of $\mathcal{M} \in \mathbf{S}_{n}\left(\mathcal{A}_{i}^{\prime}, H_{i, j}^{\prime}\right)$ for which $G^{\prime} \cong \operatorname{Aut}(\mathcal{M})$ converges to 1 . Then

$$
\frac{\left|\left\{\mathcal{M} \in \mathbf{S}_{n}: G^{\prime} \cong \operatorname{Aut}(\mathcal{M})\right\}\right|}{\left|\left\{\mathcal{M} \in \mathbf{S}_{n}: G \cong \operatorname{Aut}(\mathcal{M})\right\}\right|} \sim \frac{\left|\bigcup_{i=1}^{m^{+}} \bigcup_{j=1}^{l_{i}^{+}} \mathbf{S}_{n}\left(\mathcal{A}_{i}^{+}, H_{i, j}^{+}\right)\right|}{\left|\bigcup_{i=1}^{m^{*}} \bigcup_{j=1}^{l_{i}^{*}} \mathbf{S}_{n}\left(\mathcal{A}_{i}^{*}, H_{i, j}^{*}\right)\right|}
$$

where, by Proposition 5.9, the right side converges to a rational number or tends to infinity as $n \rightarrow \infty$.

Remark 5.17 By the use of Lemma 4.3 and arguments similar to those already carried out one can prove that $a_{n} / b_{n}$ converges to a rational number as $n \rightarrow \infty$ if, for example, $a_{n}=\left|\mathbf{S}_{n}\left(\mathrm{spt} \geq k_{1}\right)\right|$ and $b_{n}=\left|\mathbf{S}_{n}\left(\mathrm{spt} \geq k_{2}\right)\right|$.

\section{Logical limit laws}

The main results of this section are Theorems 6.1 and 6.2, where the later implies Theorem 1.2. In Remark 6.9 we observe that we do not have a zero-one law in Theorems 6.2 or 1.2 .

Theorem 6.1 Suppose that $\mathcal{A} \in \mathbf{S}$ has no fixed point and let $H$ be a subgroup of $\operatorname{Aut}(\mathcal{A})$ without any fixed point. Then $\mathbf{S}(\mathcal{A}, H)$ has a zero-one law.

Before proving Theorem 6.1 we derive:

Theorem 6.2 (i) Let $\mathcal{A}_{1}, \ldots, \mathcal{A}_{m} \in \mathbf{S}$ be such that none of them has any fixed point. Suppose that for every $i=1, \ldots, m$ and $j=1, \ldots, l_{i}, H_{i, j}$ is a subgroup of $\operatorname{Aut}\left(\mathcal{A}_{i}\right)$ without any fixed point. Then $\bigcup_{i=1}^{m} \bigcup_{j=1}^{l_{i}} \mathbf{S}\left(\mathcal{A}_{i}, H_{i, j}\right)$ has a limit law. Moreover, there is a finite set $Q$ of rational numbers that depends only on the $\mathcal{A}_{i}$ and $H_{i, j}$ such that for every 
sentence $\varphi$, the proportion of $n$-element structures in the double union in which $\varphi$ is true converges to a number in $Q$ as $n \rightarrow \infty$.

(ii) The following sets have limit laws: for every finite group $G,\{\mathcal{M} \in \mathbf{S}: G \cong$ $\operatorname{Aut}(\mathcal{M})\}$ and $\{\mathcal{M} \in \mathbf{S}: G \leq \operatorname{Aut}(\mathcal{M})\}$, and for every integer $m \geq 2, \mathbf{S}\left(\mathrm{spt}^{*}=m\right)$, $\mathbf{S}(\mathrm{spt} \geq m)$ and $\mathbf{S}\left(\mathrm{spt}^{*} \geq m\right)$. Moreover, in each case there is a finite set $Q \subseteq \mathbb{Q}$ such that for every sentence $\varphi$, the proportion of $n$-element structures in which $\varphi$ is true converges to a number in $Q$ as $n \rightarrow \infty$.

Proof Part (i) is immediate from Theorem 6.1 and Proposition 5.9. For part (ii) let $\mathbf{X}$ be any one of the sets of structures considered. By Lemmas 4.2 and 4.3 (and in one case the proof of Proposition 5.15), there are structures $\mathcal{A}_{1}, \ldots, \mathcal{A}_{l} \in \mathbf{S}$ without any fixed point and for every $i=1, \ldots, l$ and $j=1, \ldots, l_{i}$, a subgroup $H_{i, j}$ of $\operatorname{Aut}\left(\mathcal{A}_{i}\right)$ without any fixed point, such that if $\mathbf{X}_{n}=\mathbf{X} \cap \mathbf{S}_{n}$ then

$$
\left|\mathbf{X}_{n}\right| \sim\left|\bigcup_{i=1}^{l} \bigcup_{j=1}^{l_{i}} \mathbf{S}_{n}\left(\mathcal{A}_{i}, H_{i, j}\right)\right| .
$$

Now part (ii) follows from part (i).

\subsection{Proof of Theorem 6.1}

Suppose that $\mathcal{A} \in \mathbf{S}$ has no fixed point and let $H$ be a subgroup of $\operatorname{Aut}(\mathcal{A})$ without any fixed point. We will define a theory $T_{\mathcal{A}, H}$ and show that it is consistent and complete and that for every finite subset $\Delta \subseteq T_{\mathcal{A}, H}$, the proportion of $\mathcal{M} \in \mathbf{S}_{n}(\mathcal{A}, H)$ such that $\mathcal{M} \models \Delta$ approaches 1 as $n \rightarrow \infty$. Then the compactness theorem implies that if $T_{\mathcal{A}, H}=\varphi$ then the proportion of $\mathcal{M} \in \mathbf{S}_{n}(\mathcal{A}, H)$ in which $\varphi$ is true approaches 1 as $n \rightarrow \infty$; otherwise that proportion approaches 0 . The argument follows a well known path, using so-called extension axioms. What makes things more complicated here, compared with Fagin's original proof Fagin ([8] or Ebbinghaus and Flum [6]) that for every $k \in \mathbb{N}$ the proportion of $\mathcal{M} \in \mathbf{S}_{n}$ satisfying the $k$-extension axiom approaches 1 as $n \rightarrow \infty$, is that all members of $\mathbf{S}_{n}(\mathcal{A}, H)$ have nonempty support (of cardinality $|A|$ ). We will define $T_{\mathcal{A}, H}$ to consist of a sentence $\psi$ implying that the support is isomorphic to $\mathcal{A}$ and, for each $k \in \mathbb{N}$, a $k$-extension axiom $\varphi_{k}$ which takes the support in careful consideration.

To make the main ideas of the argument more transparent, to avoid heavy formulations and notation and to expose more clearly how our argument differs from the "standard argument" (in $[6,8]$ for example), we will prove Theorem 6.1 in the special case when 
the vocabulary consists of only one binary relation symbol $R$. It is straightforward to generalise the argument to any finite relational vocabulary with at least one relation symbol of arity at least 2, but it comes at the expense of longer definitions and heavier notation and formulations in order to keep track of all data and how it can be combined. Moreover, the arguments can be modified to the case when we assume that some (possibly all) relation symbols are always interpreted as irreflexive relations, or when we assume that some (possibly all) relation symbols are always interpreted as irreflexive and symmetric relations.

For any structure $\mathcal{M}$ and formula $\varphi(x)$ we let

$$
\varphi(\mathcal{M})=\{a \in M: \mathcal{M} \models \varphi(a)\} .
$$

Let $p=|A|, A=\left\{a_{1}, \ldots, a_{p}\right\}$ and let $x_{1}, \ldots, x_{p}$ be distinct variables. For $a_{i}, a_{j} \in A$, let $a_{i} \approx a_{j}$ mean that $a_{i}$ and $a_{j}$ belong to the same orbit of $H$. Let $\chi_{\mathcal{A}}\left(x_{1}, \ldots, x_{p}\right)$ be a formula which describes the isomorphism type of $\mathcal{A}$. More precisely, $\chi_{\mathcal{A}}\left(x_{1}, \ldots, x_{p}\right)$ is the conjunction of all formulas of the following form: $x_{i} \neq x_{j}$ for $i \neq j ; R\left(x_{i}, x_{j}\right)$ if $\mathcal{A} \models R\left(a_{i}, a_{j}\right)$; and $\neg R\left(x_{i}, x_{j}\right)$ if $\mathcal{A} \models \neg R\left(a_{i}, a_{j}\right)$.

We will define formulas $\theta(x), \xi(x, y)$ such that the proportion of $\mathcal{M} \in \mathbf{S}_{n}(\mathcal{A}, H)$ such that the following hold approaches 1 as $n \rightarrow \infty$ :

(I) For every $a \in M, \mathcal{M} \models \theta(a)$ if and only if $a \in \operatorname{Spt}^{*}(\mathcal{M})$.

(II) $\mathcal{M}$ satisfies the following sentence, denoted by $\psi$ :

$$
\begin{aligned}
& \exists x_{1}, \ldots, x_{m}\left(\chi_{\mathcal{A}}\left(x_{1}, \ldots, x_{m}\right) \wedge \forall y\left[\theta(y) \longleftrightarrow \bigvee_{i=1}^{m} y=x_{i}\right] \wedge\right. \\
& \bigwedge_{a_{i} \approx a_{j}} \xi\left(x_{i}, x_{j}\right) \wedge \bigwedge_{a_{i} \approx a_{j}} \neg \xi\left(x_{i}, x_{j}\right) \wedge \\
& \bigwedge_{i, j=1}^{m} \forall y\left[\neg \theta(y) \wedge \xi\left(x_{i}, x_{j}\right) \longrightarrow\right. \\
&\left.\left.\left(\xi\left(y, x_{i}\right) \longleftrightarrow \xi\left(y, x_{j}\right)\right) \wedge\left(\xi\left(x_{i}, y\right) \longleftrightarrow \xi\left(x_{j}, y\right)\right)\right]\right) .
\end{aligned}
$$

Then $\psi$ says that there exists a copy of $\mathcal{A}$ whose universe is defined by $\theta$ and whose orbit equivalence relation is defined by $\xi$. It is straightforward to define, for every $k \in \mathbb{N}$, a sentence $\varphi_{k}$ such that for every, possibly infinite, structure $\mathcal{M}$ :

(III) If $\mathcal{M}=\varphi_{k}$ then the following hold:

(a) $|\theta(\mathcal{M})|=m$ and the relation defined by $\xi(x, y)$ restricted to $\theta(\mathcal{M})$ is an equivalence relation. 
(b) For every choice of $i \in\{0,1\}, B \subseteq M \backslash \theta(\mathcal{M})$ with $|B|=k$, sets $E, E^{\prime}$ of $\xi$-equivalence classes on $\theta(\mathcal{M})$ and $Y, Y^{\prime} \subseteq B$, there is $c \in M \backslash \theta(\mathcal{M})$ such that

(i) $\mathcal{M}=R(c, c) \Longleftrightarrow i=1$,

(ii) for every $a \in \theta(\mathcal{M})$,

$\mathcal{M} \models R(c, a) \Longleftrightarrow a$ belongs to some class in $E$ and

$\mathcal{M} \models R(a, c) \Longleftrightarrow a$ belongs to some class in $E^{\prime}$, and

(iii) for every $b \in B$,

$$
\begin{aligned}
& \mathcal{M}=R(c, b) \Longleftrightarrow b \in Y \text { and } \\
& \mathcal{M}=R(b, c) \Longleftrightarrow b \in Y^{\prime} .
\end{aligned}
$$

We call $\varphi_{k}$ the $k$-extension axiom. Assuming that we have $\theta(x), \xi(x, y), \psi$ and $\varphi_{k}$, $k \in \mathbb{N}$, as above, we let

$$
T_{\mathcal{A}, H}=\{\psi\} \cup\left\{\varphi_{k}: k \in \mathbb{N}\right\} .
$$

Note that every model of $T_{\mathcal{A}, H}$ is infinite. We postpone the proof that $T_{\mathcal{A}, H}$ has a model to the end of the argument. By Łos' and Vaught's categoricity theorem (see Rothmaler [19, Theorem 8.5.1], for instance), $T_{\mathcal{A}, H}$ is complete if we can prove that it is countably categorical.

Lemma 6.3 If $\mathcal{M}_{1}$ and $\mathcal{M}_{2}$ are countable models of $T_{\mathcal{A}, H}$ then $\mathcal{M}_{1} \cong \mathcal{M}_{2}$.

Proof This is a standard back-and-forth argument, so we only sketch it. Suppose that $\mathcal{M}_{1}$ and $\mathcal{M}_{2}$ are countable models of $T_{\mathcal{A}, H}$. Since both $\mathcal{M}_{1}$ and $\mathcal{M}_{2}$ satisfy $\psi$ it follows that $\theta\left(\mathcal{M}_{1}\right)$ and $\theta\left(\mathcal{M}_{2}\right)$ are finite and that there is an isomorphism $f_{0}: \mathcal{M}_{1} \uparrow \theta\left(\mathcal{M}_{1}\right) \rightarrow \mathcal{M}_{2}\left\lceil\theta\left(\mathcal{M}_{2}\right)\right.$ such that for all $a, b \in \theta\left(\mathcal{M}_{1}\right), \mathcal{M}_{1} \models \xi(a, b)$ if and only if $\mathcal{M}_{2} \models \xi\left(f_{0}(a), f_{0}(b)\right)$. Therefore it suffices to prove the following statement:

Claim Suppose that $B_{1} \subseteq M_{1} \backslash \theta\left(\mathcal{M}_{1}\right), B_{2} \subseteq M_{2} \backslash \theta\left(\mathcal{M}_{2}\right)$ and that

$$
f: \mathcal{M}_{1} \uparrow\left(\theta\left(\mathcal{M}_{1}\right) \cup B_{1}\right) \rightarrow \mathcal{M}_{2}\left\lceil\left(\theta\left(\mathcal{M}_{2}\right) \cup B_{2}\right)\right.
$$

is an isomorphism. If $c_{1} \in M_{1} \backslash\left(\theta\left(\mathcal{M}_{1}\right) \cup B_{1}\right)$ (or $c_{2} \in M_{2} \backslash\left(\theta\left(\mathcal{M}_{2}\right) \cup B_{2}\right)$ ) then there is $c_{2} \in M_{2} \backslash\left(\theta\left(\mathcal{M}_{2}\right) \cup B_{2}\right)$ (or $c_{1} \in M_{1} \backslash\left(\theta\left(\mathcal{M}_{1}\right) \cup B_{1}\right)$ ) such that $f$ can be extended to an isomorphism from $\mathcal{M}_{1}\left\lceil\left(\theta\left(\mathcal{M}_{1}\right) \cup B_{1} \cup\left\{c_{1}\right\}\right)\right.$ to $\mathcal{M}_{2}\left\lceil\left(\theta\left(\mathcal{M}_{2}\right) \cup B_{2} \cup\left\{c_{2}\right\}\right)\right.$.

Let $k=\left|B_{1}\right|=\left|B_{2}\right|$. The claim follows in a straightforward way since $\mathcal{M}$ and $\mathcal{N}$ are models of $\left\{\psi, \varphi_{k}\right\}$. 
It remains to show that there are $\theta(x)$ and $\xi(x, y)$ such that, for every $k$, the proportion of $\mathcal{M} \in \mathbf{S}_{n}(\mathcal{A}, H)$ that satisfy (I) and the sentences $\psi$ and $\varphi_{k}$ approaches 1 as $n \rightarrow \infty$. Recall that, with the notation from Section 4,

$$
\mathbf{S}_{n}(\mathcal{A}, H)=\bigcup_{X} \bigcup_{\mathcal{A}_{X}} \mathbf{S}_{X}\left(\mathcal{A}_{X}, H\right),
$$

where the first union ranges over all subsets of $[n]$ with cardinality $m=|A|$, and for each such subset $X$, the second union ranges over all structures $\mathcal{A}_{X}$ with universe $X$ that are isomorphic to $\mathcal{A}$. As observed in that section, if $X \neq X^{\prime}$ then $\mathbf{S}_{n}\left(\mathcal{A}_{X}, H\right)$ is disjoint from $\mathbf{S}_{n}\left(\mathcal{A}_{X^{\prime}}, H\right)$. Moreover, if $\mathcal{A}_{X}$ and $\mathcal{A}_{X}^{\prime}$ are different structures with universe $X$ then $\mathbf{S}_{n}\left(\mathcal{A}_{X}, H\right)$ is disjoint from $\mathbf{S}_{n}\left(\mathcal{A}_{X}^{\prime}, H\right)$. Recall our assumption in this proof that there is only one relation symbol $R$ and it has arity $r=2$. In Section 4 we also saw (recall (4-4)) that for each $\mathbf{S}_{n}\left(\mathcal{A}_{X}, H\right)$,

$$
\mathbf{S}_{n}\left(\mathcal{A}_{X}, H\right)=\bigcup_{\Pi_{1}} \mathbf{S}_{n}\left(\mathcal{A}_{X}, \Pi_{1}\right)
$$

where the union ranges over all $\left(\mathcal{A}_{X}, H\right)$-partitions $\Pi_{1}$ of $X$; see Definition 4.8. The number of $\left(\mathcal{A}_{X}, H\right)$-partitions of $X$ is the same for every sufficiently large $n$, every $X \subseteq[n]$ with $|X|=m$ and every $\mathcal{A}_{X} \cong \mathcal{A}$. Therefore it suffices to prove that there are $\theta(x), \xi(x, y)$ and, for every $k, 0<\alpha<1$ such that for every $X \subseteq[n]$ with $|X|=m$, every $\mathcal{A}_{X} \cong \mathcal{A}$ with universe $X$ and every $\left(\mathcal{A}_{X}, H\right)$-partition $\Pi_{1}$, the proportion of $\mathcal{M} \in \mathbf{S}_{n}\left(\mathcal{A}_{X}, \Pi_{1}\right)$ that satisfy (I) and the sentences $\psi$ and $\varphi_{k}$ is at least $1-\alpha^{n}$.

For the rest of this section we fix $X \subseteq[n]$ with $|X|=|A|=m$ and $\mathcal{A}_{X} \cong \mathcal{A}$ with universe $X$. The results below refer to all large enough $n$ with respect to other parameters that occur.

Definition 6.4 We say that $\mathcal{M} \in \mathbf{S}_{n}\left(\mathcal{A}_{X}, \Pi_{1}\right)$ has the $k$-extension property if (III) (b) holds when ' $\theta(\mathcal{M})$ ' is replaced by ' $X$ ' and ' $\xi$-equivalence classes' with 'parts of the partition $\Pi_{1}(\text { of } X)^{\prime}$.

Lemma 6.5 For every $k \in \mathbb{N}$ there is $0<\alpha_{k}<1$, depending only on $k$ and $\mathcal{A}$, such that the proportion of $\mathcal{M} \in \mathbf{S}_{n}\left(\mathcal{A}_{X}, \Pi_{1}\right)$ which does not have the $k$-extension property is at most $\alpha_{k}^{n}$.

Proof Recall that $\mathbf{S}_{n}\left(\mathcal{A}_{X}, \Pi_{1}\right) \subseteq \mathbf{T}_{n}\left(\mathcal{A}_{X}, \Pi_{1}\right)$, where $\mathbf{T}_{n}\left(\mathcal{A}_{X}, \Pi_{1}\right)$ was defined in (4-3) in Section 4. From Lemma 4.9 we know that $\left|\mathbf{S}_{n}\left(\mathcal{A}_{X}, \Pi_{1}\right)\right| /\left|\mathbf{T}_{n}\left(\mathcal{A}_{X}, \Pi_{1}\right)\right| \rightarrow 1$ as $n \rightarrow \infty$, so it suffices to prove the statement of the lemma for $\mathbf{T}_{n}\left(\mathcal{A}_{X}, \Pi_{1}\right)$ in the place of $\mathbf{S}_{n}\left(\mathcal{A}_{X}, \Pi_{1}\right)$. The reason for doing this is that $\mathbf{T}_{n}\left(\mathcal{A}_{X}, \Pi_{1}\right)$ is easier to work with 
because its members do not have the constraint that the support of the structure is exactly $X$ (but from the arguments in Section 4 we know that for every $\mathcal{M} \in \mathbf{T}_{n}\left(\mathcal{A}_{X}, \Pi_{1}\right)$, $\left.X \subseteq \operatorname{Spt}^{*}(\mathcal{M})\right)$.

Let $\mathcal{M} \in \mathbf{T}_{n}\left(\mathcal{A}_{X}, \Pi_{1}\right)$. A subset $B \subseteq[n] \backslash X$ of cardinality $k$ can be chosen in no more than $n^{k}$ ways. Once $B \subseteq[n] \backslash X$ with $|B|=k$ is fixed, the number of ways to choose $i \in\{0,1\}, E, E^{\prime} \subseteq \Pi_{1}$ and $Y, Y^{\prime} \subseteq B$ is bounded where the bound depends only on $k$ and $\mathcal{A}$. Therefore it suffices to show, for an arbitrary fixed $B \subseteq[n] \backslash X$ with $|B|=k$ and an arbitrary choice of $i \in\{0,1\}, E, E^{\prime} \subseteq \Pi_{1}$ and $Y, Y^{\prime} \subseteq B$, that the proportion of $\mathcal{M} \in \mathbf{T}_{n}\left(\mathcal{A}_{X}, \Pi_{1}\right)$ such that there is no $c \in M$ such that the conjunction of (i)-(iii) of (III) is satisfied is at most $\alpha_{k}^{n}$ for some constant $0<\alpha_{k}<1$ that depends only on $k$ and $\mathcal{A}$.

For arbitrary $c \in[n] \backslash(X \cup B)$ we estimate the probability that at least one of (i)-(iii) of (III) fails. We consider $\mathbf{T}_{n}\left(\mathcal{A}_{X}, \Pi_{1}\right)$ as a probability space by giving each member the same probability. From the definition of $\mathbf{T}_{n}\left(\mathcal{A}_{X}, \Pi_{1}\right)$ we see that the probability that $\mathcal{M} \in \mathbf{T}_{n}\left(\mathcal{A}_{X}, \Pi_{1}\right)$ satisfies (i)-(iii) of (III) is $2^{-\beta}$ for some $\beta>0$ depending only on $|X|$ and $|B|$, and independently of what the case is for other elements than $c$ in $[n] \backslash(X \cup B)$. The probability that, for every $c \in[n] \backslash(X \cup B)$, the conjunction of (i)-(iii) does not hold is therefore

$$
\left(1-2^{-\beta}\right)^{n-|X \cup B|} \text {. }
$$

As $B$ can be chosen in at most $n^{k}$ ways it follows that the probability that the conjunction of (i)-(iii) is not satisfied in $\mathcal{M} \in \mathbf{T}_{n}\left(\mathcal{A}_{X}, \Pi_{1}\right)$ is at most $\alpha_{k}^{n}$ for some $0<\alpha_{k}<1$ that depends only on $k$ and $\mathcal{A}$.

Remember that $m=|A|=|X|$. Let $\theta(x)$ denote the following formula:

$$
\begin{aligned}
& \exists y_{1}, \ldots, y_{m-1}\left(\bigwedge_{i=1}^{m-1} x \neq y_{i} \wedge \bigwedge_{i \neq j} y_{i} \neq y_{j} \wedge\right. \\
&\left.\forall z\left[\left(z \neq x \wedge \bigwedge_{i=1}^{m-1} z \neq y_{i}\right) \longrightarrow\left(R(x, z) \longleftrightarrow R\left(y_{1}, z\right)\right)\right]\right) .
\end{aligned}
$$

Lemma 6.6 Suppose that $\mathcal{M} \in \mathbf{S}_{n}\left(\mathcal{A}_{X}, \Pi_{1}\right)$ has the 2 -extension property. Then for all $a \in M, a \in X=\operatorname{Spt}^{*}(\mathcal{M})$ if and only if $\mathcal{M}=\theta(a)$.

Proof Suppose that $\mathcal{M}=\theta(a)$. Then there are distinct $b_{1}, \ldots, b_{m-1} \in M$ different from $a$ such that for all $c$ different from $b_{1}, \ldots, b_{m-1}$ and from $a$,

$$
\mathcal{M} \models R(a, c) \longleftrightarrow R\left(b_{1}, c\right)
$$


As $\mathcal{M}$ has the 2-extension property this is only possible if $a, b_{1} \in X=\operatorname{Spt}^{*}(\mathcal{M})$ and $a$ and $b_{1}$ belong to the same part of $\Pi_{1}$.

Now suppose that $a \in \operatorname{Spt}^{*}(\mathcal{M})=X$. Let $b_{1}, \ldots, b_{m-1}$ be such that

$$
X=\left\{a, b_{1}, \ldots, b_{m-1}\right\}
$$

and $a$ and $b_{1}$ belong to the same part of $\Pi_{1}$. By the definition of $\mathbf{S}_{n}\left(\mathcal{A}_{X}, \Pi_{1}\right)$, there is an automorphism of $\mathcal{M}$ which sends $a$ to $b_{1}$ and fixes every element outside of $X$ and therefore we must have

$$
\mathcal{M} \models \forall z\left[\left(z \neq a \wedge \bigwedge_{i=1}^{m-1} z \neq b_{i}\right) \longrightarrow\left(R(a, z) \longleftrightarrow R\left(b_{1}, z\right)\right)\right] .
$$

Let $\xi\left(x_{1}, x_{2}\right)$ be the formula

$$
\forall z\left(\neg \theta(z) \longrightarrow\left[R\left(z, x_{1}\right) \longleftrightarrow R\left(z, x_{2}\right)\right]\right) .
$$

Lemma 6.7 Suppose that $\mathcal{M} \in \mathbf{S}_{n}\left(\mathcal{A}_{X}, \Pi_{1}\right)$ has the 2-extension property. Then for all $a_{1}, a_{2} \in X=\operatorname{Spt}^{*}(\mathcal{M}), a_{1}$ and $a_{2}$ belong to the same part of $\Pi_{1}$ if and only if $\mathcal{M}=\xi\left(a_{1}, a_{2}\right)$.

Proof Suppose that $a_{1}, a_{2} \in X=\operatorname{Spt}^{*}(\mathcal{M})$ and $a_{1}$ and $a_{2}$ belong to the same part of $\Pi_{1}$. By the definition of $\mathbf{S}_{n}\left(\mathcal{A}_{X}, \Pi_{1}\right)$, for every $c \in M \backslash X$ there is an automorphism which sends $a_{1}$ to $a_{2}$ and fixes every element outside of $X$. From Lemma 6.6 it follows that $\mathcal{M}=\xi\left(a_{1}, a_{2}\right)$.

Now suppose that $a_{1}, a_{2} \in X=\operatorname{Spt}^{*}(\mathcal{M})$ and $\mathcal{M} \models \xi\left(a_{1}, a_{2}\right)$. From Lemma 6.6 it follows that for all $c \in M \backslash X$

$$
\mathcal{M} \models R\left(c, a_{1}\right) \Longleftrightarrow R\left(c, a_{2}\right) .
$$

Since we assume that $\mathcal{M}$ has the 2 -extension property this is only possible if $a_{1}$ and $a_{2}$ belong to the same part of $\Pi_{1}$.

According to the arguments before Definition 6.4 and the compactness theorem, the following corollary concludes the proof of Theorem 6.1 .

Corollary 6.8 For every $k \in \mathbb{N}$, there is $0<\alpha<1$, depending only on $k$ and $\mathcal{A}$, such that the proportion of $\mathcal{M} \in \mathbf{S}_{n}\left(\mathcal{A}_{X}, \Pi_{1}\right)$ that satisfy (I) and the sentences $\psi$ and $\varphi_{l}$ for $l=0, \ldots, k$ is at least $1-\alpha^{n}$. 
Proof Let $k^{\prime}=\max (2, k, m)$ (where $m=|A|$ ). By Lemma 6.5 there is $0<\alpha<1$, depending only on $k^{\prime}$ and $\mathcal{A}$ such that the proportion of $\mathcal{M} \in \mathbf{S}_{n}\left(\mathcal{A}_{X}, \Pi_{1}\right)$ with the $l$-extension property for every $l \leq k^{\prime}$ is at least $1-\alpha^{n}$. From Lemmas 6.6 and 6.7 it follows that all $\mathcal{M} \in \mathbf{S}_{n}\left(\mathcal{A}_{X}, \Pi_{1}\right)$ with the $l$-extension property for every $l \leq k^{\prime}$ satisfy (I) and the sentences $\psi$ and $\varphi_{l}$ for $l=0, \ldots, k$.

Remark 6.9 Let $\mathbf{S}^{\prime}$ be any one of the sets of structures in part (ii) of Theorem 6.2 and let $\mathbf{S}_{n}^{\prime}=\mathbf{S}^{\prime} \cap \mathbf{S}_{n}$. We assume that if a finite group $G$ is involved in the definition of $\mathbf{S}^{\prime}$ then $G$ is nontrivial. We will show that $\mathbf{S}^{\prime}$ does not satisfy a zero-one law. By Lemmas 4.2 and 4.3 (and in one case the proof of Proposition 5.15), there are mutually nonisomorphic $\mathcal{A}_{1}, \ldots, \mathcal{A}_{l} \in \mathbf{S}$ without any fixed point and, for $i=1, \ldots, l$ and $j=1, \ldots, l_{i}$, subgroups $H_{i, j} \subseteq \operatorname{Aut}\left(\mathcal{A}_{i}\right)$ without any fixed point such that

$$
\left|\mathbf{S}_{n}^{\prime}\right| \sim\left|\bigcup_{i=1}^{l} \bigcup_{j=1}^{l_{i}} \mathbf{S}_{n}\left(\mathcal{A}_{i}, H_{i, j}\right)\right| .
$$

If $\mathbf{S}^{\prime}$ is $\{\mathcal{M} \in \mathbf{S}: G \leq \operatorname{Aut}(\mathcal{M})\}$ or $\{\mathcal{M} \in \mathbf{S}: G \cong \operatorname{Aut}(\mathcal{M})\}$, then we may also assume that $G \leq H_{i, j}$ or $G \cong H_{i, j}$, respectively, for all $i$ and $j$.

Now observe the following: Suppose that $\mathcal{A} \in \mathbf{S}$ has no fixed point and that $H$ is a subgroup of $\operatorname{Aut}(\mathcal{A})$ without any fixed point. Let $\mathcal{A}^{\prime}$ and $\mathcal{A}^{\prime \prime}$ have the same universe $A$ as $\mathcal{A}$ and assume that for every relation symbol $R, R^{\mathcal{A}^{\prime}}=\emptyset$ and $R^{\mathcal{A}^{\prime \prime}}=A^{i}$ if $R$ is $i$-ary. Then $H$ is a subgroup of $\operatorname{Aut}\left(\mathcal{A}^{\prime}\right)$ and of $\operatorname{Aut}\left(\mathcal{A}^{\prime \prime}\right)$ and, from Proposition 4.4, it follows that

$$
\frac{\left|\mathbf{S}_{n}\left(\mathcal{A}^{\prime}, H\right)\right|}{\left|\mathbf{S}_{n}(\mathcal{A}, H)\right|} \quad \text { and } \quad \frac{\left|\mathbf{S}_{n}\left(\mathcal{A}^{\prime \prime}, H\right)\right|}{\left|\mathbf{S}_{n}(\mathcal{A}, H)\right|}
$$

converge to the same $c \in \mathbb{Q}$ as $n \rightarrow \infty$. From the assumption that $\mathbf{S}^{\prime}$ is one of the sets of structures in part (ii) of Theorem 6.2 (and $G$ is assumed to be nontrivial) it follows that there must be $i, i^{\prime}, j, j^{\prime}$ such that $\mathcal{A}_{i} \not \mathcal{A}_{i^{\prime}}$ and both $\left|\mathbf{S}_{n}\left(\mathcal{A}_{i}, H_{i, j}\right)\right| /\left|\mathbf{S}_{n}^{\prime}\right|$ and $\left|\mathbf{S}_{n}\left(\mathcal{A}_{i^{\prime}}, H_{i^{\prime}, j^{\prime}}\right)\right| /\left|\mathbf{S}_{n}^{\prime}\right|$ converge to positive numbers $c$ and $c^{\prime}$ as $n \rightarrow \infty$. With the help of the formula $\theta$ from the proof of Theorem 6.1 one can easily construct a sentence $\varphi$ which, in almost all $\mathcal{M} \in \mathbf{S}^{\prime}$, expresses that " $\mathcal{M}\left\lceil\operatorname{Spt}^{*}(\mathcal{M}) \cong \mathcal{A}_{i}\right.$ ". Then the proportion of $\mathcal{M} \in \mathbf{S}_{n}^{\prime}$ in which $\varphi$ is true converges to some number $0<d<1$.

\section{Unlabelled structures}

The main result of this final section is Theorem 7.7, which implies Theorem 1.3, which says that Theorems 1.1 and 1.2 hold also for unlabelled structures. 
Definition 7.1 (i) For every $\mathcal{M} \in \mathbf{S}$, let $[\mathcal{M}]=\{\mathcal{N} \in \mathbf{S}: \mathcal{N} \cong \mathcal{M}\}$.

(ii) For every $\mathbf{X} \subseteq \mathbf{S}$, let $[\mathbf{X}]=\{[\mathcal{M}]: \mathcal{M} \in \mathbf{X}\}$.

(iii) We say that a set $\mathbf{X} \subseteq \mathbf{S}$ is closed under isomorphism if $\mathcal{M} \in \mathbf{X}, \mathcal{N} \in \mathbf{S}$ and $\mathcal{N} \cong \mathcal{M}$ implies that $\mathcal{N} \in \mathbf{X}$.

The next lemma is a generalisation of Lemma 4.3.10 in Ebbinghaus and Flum [6].

Lemma 7.2 If $\mathbf{X}_{n} \subseteq \mathbf{S}_{n}$ is closed under isomorphism then

$$
\left|\left[\mathbf{X}_{n}\right]\right| n !=\sum_{\pi \in \text { Sym }_{n}}\left|\mathbf{S}_{n}(\pi) \cap \mathbf{X}_{n}\right|
$$

Proof For every $\mathcal{M} \in \mathbf{S}_{n}$ and $\pi \in \operatorname{Sym}_{n}$, let $\pi(\mathcal{M})$ denote the unique structure $\mathcal{N} \in \mathbf{S}_{n}$ such that $\pi$ is an isomorphism from $\mathcal{M}$ onto $\mathcal{N}$. Fix an arbitrary $\mathcal{M} \in \mathbf{X}_{n}$ and let $H=\operatorname{Aut}(\mathcal{M})$. Then $H$ is a subgroup of $S_{y m}$ and we consider the left cosets of $H$ in $S_{y m}$. Note that for every $\mathcal{N} \in \mathbf{X}_{n}$ we have $\mathcal{N} \cong \mathcal{M}$ if and only if there is $\pi \in \operatorname{Sym}_{n}$ such that $\pi(\mathcal{M})=\mathcal{N}$. For all $\pi, \sigma \in \operatorname{Sym}_{n}$ we have

$$
\pi H=\sigma H \Longleftrightarrow H=\pi^{-1} \sigma H \Longleftrightarrow \pi^{-1} \sigma \in H=\operatorname{Aut}(\mathcal{M}) \Longleftrightarrow \pi(\mathcal{M})=\sigma(\mathcal{M})
$$

As we assume that $\mathbf{X}_{n}$ is closed under isomorphism it follows that

$$
\left|\left\{\mathcal{N} \in \mathbf{X}_{n}: \mathcal{N} \cong \mathcal{M}\right\}\right|=\text { the number of cosets (the index) of } \operatorname{Aut}(\mathcal{M}) \text { in } \operatorname{Sym}_{n} .
$$

Hence

$$
|\operatorname{Aut}(\mathcal{M})| \cdot\left|\left\{\mathcal{N} \in \mathbf{X}_{n}: \mathcal{N} \cong \mathcal{M}\right\}\right|=\left|\operatorname{Sym}_{n}\right|=n !,
$$

and, as $|\operatorname{Aut}(\mathcal{N})|=|\operatorname{Aut}(\mathcal{M})|$ if $\mathcal{N} \cong \mathcal{M}$, we get

$$
\sum_{\substack{\mathcal{N} \in \mathbf{X}_{n} \\ \mathcal{N} \cong \mathcal{M}}}|\operatorname{Aut}(\mathcal{N})|=\sum_{\substack{\mathcal{N} \in \mathbf{X}_{n} \\ \mathcal{N} \cong \mathcal{M}}}|\operatorname{Aut}(\mathcal{M})|=\left|\left\{\mathcal{N} \in \mathbf{X}_{n}: \mathcal{N} \cong \mathcal{M}\right\}\right| \cdot|\operatorname{Aut}(\mathcal{M})|=n !
$$

If $\mathcal{M}_{1}, \ldots, \mathcal{M}_{m}$ is a sequence containing exactly one representative from every isomorphism class that is represented in $\mathbf{X}_{n}$, then $m=\left|\left[\mathbf{X}_{n}\right]\right|$ and

$$
\sum_{\mathcal{M} \in \mathbf{X}_{n}}|\operatorname{Aut}(\mathcal{M})|=\sum_{i=1}^{m} \sum_{\substack{\mathcal{N} \in \mathbf{X}_{n} \\ \mathcal{N} \cong \mathcal{M}_{i}}}|\operatorname{Aut}(\mathcal{N})|=\sum_{i=1}^{m} n !=\left|\left[\mathbf{X}_{n}\right]\right| \cdot n !
$$

We also have

$\sum_{\mathcal{M} \in \mathbf{X}_{n}}|\operatorname{Aut}(\mathcal{M})|=\mid\left\{(\mathcal{M}, \pi): \mathcal{M} \in \mathbf{X}_{n}\right.$ and $\left.\pi \in \operatorname{Aut}(\mathcal{M})\right\}\left|=\sum_{\pi \in \text { Sym }_{n}}\right| \mathbf{S}_{n}(\pi) \cap \mathbf{X}_{n} \mid$,

which concludes the proof of the lemma. 
Lemma 7.3 If $\mathbf{Y} \subseteq \mathbf{S}$ is closed under isomorphism and $p \geq 2$ is fixed, then

$$
\left|\mathbf{S}_{n}\left(\mathrm{spt}^{*} \leq p\right) \cap \mathbf{Y}\right| \sim n !\left|\left[\mathbf{S}_{n}\left(\mathrm{spt}^{*} \leq p\right) \cap \mathbf{Y}\right]\right| \quad \text { as } n \rightarrow \infty .
$$

Proof For every permutation $\pi$ of $[n]$ and $\mathcal{M} \in \mathbf{S}_{n}$, let $\pi(\mathcal{M})$ denote the unique structure $\mathcal{M}^{\prime} \in \mathbf{S}_{n}$ such that $\pi$ is an isomorphism from $\mathcal{M}$ to $\mathcal{M}^{\prime}$. If $\mathcal{M} \in \mathbf{S}_{n}$ ( spt $^{*} \leq p$ ), $\pi$ is a permutation of $[n]$ and $\pi(\mathcal{M})=\mathcal{M}$, then $\operatorname{Spt}(\pi) \subseteq \operatorname{Spt}^{*}(\mathcal{M})$. Hence there are at most $p$ ! permutations $\pi$ of $[n]$ such that $\pi(\mathcal{M})=\mathcal{M}$. Since we assume that $\mathbf{Y}$ is closed under isomorphism we get

$$
\left|\mathbf{S}_{n}\left(\mathrm{spt}^{*} \leq p\right) \cap \mathbf{Y}\right| \geq(n !-p !)\left|\left[\mathbf{S}_{n}\left(\mathrm{spt}^{*} \leq p\right) \cap \mathbf{Y}\right]\right| .
$$

It is also clear that

$$
\left|\mathbf{S}_{n}\left(\mathrm{spt}^{*} \leq p\right) \cap \mathbf{Y}\right| \leq n !\left|\left[\mathbf{S}_{n}\left(\mathrm{spt}^{*} \leq p\right) \cap \mathbf{Y}\right]\right| .
$$

Since $(n !-p !) \sim n$ ! as $n \rightarrow \infty$, it follows that

$$
\left|\mathbf{S}_{n}\left(\mathrm{spt}^{*} \leq p\right) \cap \mathbf{Y}\right| \sim n !\left|\left[\mathbf{S}_{n}\left(\mathrm{spt}^{*} \leq p\right) \cap \mathbf{Y}\right]\right| .
$$

Proposition 7.4 Suppose that $m, t \in \mathbb{N}, f_{1}, \ldots, f_{s} \in \operatorname{Sym}_{n}, \operatorname{spt}\left(f_{1}, \ldots, f_{s}\right)=m$ and $t>2 r(m !-1) m / m !+1$, where $r \geq 2$ is the maximal arity of the relation symbols. Then there is $\lambda>0$ such that for all sufficiently large $n$,

$$
\frac{\left|\left[\mathbf{S}_{n}(\mathrm{spt} \geq t)\right]\right|}{\left|\left[\mathbf{S}_{n}\left(f_{1}, \ldots, f_{s}\right)\right]\right|} \leq 2^{-\lambda n^{r-1}}
$$

Proof Suppose that $m \in \mathbb{N}, f_{1}, \ldots, f_{s} \in \operatorname{Sym}_{n}$ and $\operatorname{spt}\left(f_{1}, \ldots, f_{s}\right)=m$. Let

$$
\widehat{\mathbf{S}}_{n}\left(f_{1}, \ldots, f_{s}\right)=\left\{\mathcal{M} \in \mathbf{S}_{n}: \mathcal{M} \cong \mathcal{N} \text { for some } \mathcal{N} \in \mathbf{S}_{n}\left(f_{1}, \ldots, f_{s}\right)\right\}
$$

and observe that $\left[\widehat{\mathbf{S}}_{n}\left(f_{1}, \ldots, f_{s}\right)\right]=\left[\mathbf{S}_{n}\left(f_{1}, \ldots, f_{s}\right)\right]$. By Propositions 2.3 and 3.5, there are constants $p, \alpha>0$ such that for all sufficiently large $n$,

$$
\frac{\left|\mathbf{S}_{n}\left(\mathrm{spt}^{*}>p\right)\right|}{\left|\mathbf{S}_{n}\left(f_{1}, \ldots, f_{s}\right)\right|} \leq 2^{-\alpha n^{r-1} \pm \mathcal{O}\left(n^{r-2}\right)}
$$

Since $\left|\mathbf{S}_{n}\left(f_{1}, \ldots, f_{s}\right)\right| \leq\left|\widehat{\mathbf{S}}_{n}\left(f_{1}, \ldots, f_{s}\right)\right|$, we get

$$
\frac{\left|\mathbf{S}_{n}\left(\mathrm{spt}^{*}>p\right)\right|}{\left|\widehat{\mathbf{S}}_{n}\left(f_{1}, \ldots, f_{s}\right)\right|} \leq 2^{-\alpha n^{r-1} \pm \mathcal{O}\left(n^{r-2}\right)},
$$

which implies that

$$
\left|\widehat{\mathbf{S}}_{n}\left(f_{1}, \ldots, f_{s}\right) \cap \mathbf{S}_{n}\left(\mathrm{spt}^{*} \leq p\right)\right| \sim\left|\widehat{\mathbf{S}}_{n}\left(f_{1}, \ldots, f_{s}\right)\right| .
$$


Lemma 7.3 with $\mathbf{Y}=\widehat{\mathbf{S}}_{n}\left(f_{1}, \ldots, f_{s}\right)$ gives

$$
\left|\widehat{\mathbf{S}}_{n}\left(f_{1}, \ldots, f_{s}\right) \cap \mathbf{S}_{n}\left(\mathrm{spt}^{*} \leq p\right)\right| \sim n !\left|\left[\widehat{\mathbf{S}}_{n}\left(f_{1}, \ldots, f_{s}\right) \cap \mathbf{S}_{n}\left(\mathrm{spt}^{*} \leq p\right)\right]\right| .
$$

This and (7-1) gives

$$
n !\left|\left[\widehat{\mathbf{S}}_{n}\left(f_{1}, \ldots, f_{s}\right) \cap \mathbf{S}_{n}\left(\mathrm{spt}^{*} \leq p\right)\right]\right| \sim\left|\widehat{\mathbf{S}}_{n}\left(f_{1}, \ldots, f_{s}\right)\right| .
$$

Suppose that $t>2 r(m !-1) m / m !+1$. By Lemma 7.2 with $\mathbf{X}_{n}=\mathbf{S}_{n}(\mathrm{spt} \geq t)$ we get

$$
\left|\left[\mathbf{S}_{n}(\mathrm{spt} \geq t)\right]\right| \cdot n !=\sum_{\pi \in \operatorname{Sym}_{n}}\left|\mathbf{S}_{n}(\pi) \cap \mathbf{S}_{n}(\mathrm{spt} \geq t)\right| .
$$

For every $\pi \in \operatorname{Sym}_{n},\left|\mathbf{S}_{n}(\pi) \cap \mathbf{S}_{n}(\mathrm{spt} \geq t)\right| \leq\left|\mathbf{S}_{n}(\mathrm{spt} \geq t)\right|$ and there are not more than $(t-1) ! n^{t-1}$ permutations $\pi \in S y m_{n}$ such that $\operatorname{spt}(\pi)<t$. Therefore,

$$
\sum_{\substack{\pi \in S y m_{n} \\ \operatorname{spt}(\pi)<t}}\left|\mathbf{S}_{n}(\pi) \cap \mathbf{S}_{n}(\mathrm{spt} \geq t)\right| \leq(t-1) ! n^{t-1}\left|\mathbf{S}_{n}(\mathrm{spt} \geq t)\right| .
$$

If $\pi \in \operatorname{Sym}_{n}$ and $\operatorname{spt}(\pi) \geq t$ then $\mathbf{S}_{n}(\pi) \cap \mathbf{S}_{n}(\operatorname{spt} \geq t)=\mathbf{S}_{n}(\pi)$, so we get

$$
\sum_{\substack{\pi \in S y m_{n} \\ \operatorname{spt}(\pi) \geq t}}\left|\mathbf{S}_{n}(\pi) \cap \mathbf{S}_{n}(\mathrm{spt} \geq t)\right|=\sum_{\substack{\pi \in S y m_{n} \\ \operatorname{spt}(\pi) \geq t}}\left|\mathbf{S}_{n}(\pi)\right| .
$$

Now we get

$$
\begin{aligned}
& \sum_{\pi \in \operatorname{Sym}_{n}}\left|\mathbf{S}_{n}(\pi) \cap \mathbf{S}_{n}(\mathrm{spt} \geq t)\right| \\
& \leq(t-1) ! n^{t-1}\left|\mathbf{S}_{n}(\mathrm{spt} \geq t)\right|+\sum_{\substack{\pi \in S_{y y m_{n}} \\
\operatorname{spt}(\pi) \geq t}}\left|\mathbf{S}_{n}(\pi)\right| \quad \text { by (7-4) and (7-5) } \\
& \leq(t-1) ! n^{t-1} \sum_{\substack{\pi \in \operatorname{Sym}_{n} \\
\operatorname{spt}(\pi) \geq t}}\left|\mathbf{S}_{n}(\pi)\right|+\sum_{\substack{\pi \in \operatorname{Sym}_{n} \\
\operatorname{spt}(\pi) \geq t}}\left|\mathbf{S}_{n}(\pi)\right| \quad \text { by (2-2) } \\
& \leq 2(t-1) ! n^{t-1} \sum_{\substack{\pi \in S y m_{n} \\
\operatorname{spt}(\pi) \geq t}}\left|\mathbf{S}_{n}(\pi)\right| \text {. }
\end{aligned}
$$


Moreover, as $\left|\left[\mathbf{S}_{n}\left(f_{1}, \ldots, f_{s}\right)\right]\right|=\left|\left[\widehat{\mathbf{S}}_{n}\left(f_{1}, \ldots, f_{s}\right)\right]\right|$ we have

$$
\begin{aligned}
& \frac{\left|\left[\mathbf{S}_{n}(\mathrm{spt} \geq t)\right]\right|}{\left|\left[\mathbf{S}_{n}\left(f_{1}, \ldots, f_{s}\right)\right]\right|}=\frac{\left|\left[\mathbf{S}_{n}(\mathrm{spt} \geq t)\right]\right|}{\left|\left[\widehat{\mathbf{S}}_{n}\left(f_{1}, \ldots, f_{s}\right)\right]\right|} \\
& \leq \frac{\left|\left[\mathbf{S}_{n}(\mathrm{spt} \geq t)\right]\right|}{\left|\left[\widehat{\mathbf{S}}_{n}\left(f_{1}, \ldots, f_{s}\right) \cap \mathbf{S}_{n}\left(\mathrm{spt}^{*} \leq p\right)\right]\right|} \\
& =\frac{n ! \cdot\left|\left[\mathbf{S}_{n}(\mathrm{spt} \geq t)\right]\right|}{\left.n ! \cdot \mid \widehat{\mathbf{S}}_{n}\left(f_{1}, \ldots, f_{s}\right) \cap \mathbf{S}_{n}\left(\mathrm{spt}^{*} \leq p\right)\right] \mid} \\
& \sim \frac{\sum_{\pi \in \text { Sym }_{n}}\left|\mathbf{S}_{n}(\pi) \cap \mathbf{S}_{n}(\mathrm{spt} \geq t)\right|}{\left|\widehat{\mathbf{S}}_{n}\left(f_{1}, \ldots, f_{s}\right)\right|} \quad \text { by (7-3) and (7-2) } \\
& \leq \frac{2(t-1) ! n^{t-1} \sum_{\substack{\pi \in S_{\operatorname{Sym}} \\
\operatorname{spt}(\pi) \geq t}}\left|\mathbf{S}_{n}(\pi)\right|}{\left|\mathbf{S}_{n}\left(f_{1}, \ldots, f_{s}\right)\right|} \quad \text { by (7-6) } \\
& \leq 2(t-1) n^{t-1} \sum_{\substack{\pi \in S y m_{n} \\
\operatorname{spt}(\pi) \geq t}} \exp _{2}\left(\sum_{i=1}^{r} k_{i} \operatorname{orb}^{i}(\pi)-\sum_{i=1}^{r} k_{i} \operatorname{orb}^{i}\left(f_{1}, \ldots, f_{s}\right)\right)
\end{aligned}
$$

by $(2-1)$ and $(2-2)$.

Since $t>2 r(m !-1) m / m !+1$ it follows from the final estimates of the proof of Proposition 2.3 that there is $\beta>0$ such that

$$
\sum_{\substack{\pi \in S y m_{n} \\ \operatorname{spt}(\pi) \geq t}} \exp _{2}\left(\sum_{i=1}^{r} k_{i} \operatorname{orb}^{i}(\pi)-\sum_{i=1}^{r} k_{i} \operatorname{orb}^{i}\left(f_{1}, \ldots, f_{s}\right)\right) \leq 2^{-\beta n^{r-1} \pm \mathcal{O}\left(n^{r-2}\right)} .
$$

This together with (7-7) implies that there is $\lambda>0$ such that

$$
\frac{\left|\left[\mathbf{S}_{n}(\mathrm{spt} \geq t)\right]\right|}{\left|\left[\mathbf{S}_{n}\left(f_{1}, \ldots, f_{s}\right)\right]\right|} \leq 2^{-\lambda n^{r-1}}
$$

for all large enough $n$.

Corollary 7.5 Let $m, t \in \mathbb{N}$.

(i) If $t>2 r(m !-1) m / m$ ! +1 then

$$
\lim _{n \rightarrow \infty} \frac{\left|\left[\mathbf{S}_{n}(\mathrm{spt} \geq t)\right]\right|}{\left|\left[\mathbf{S}_{n}(\mathrm{spt} \geq m)\right]\right|}=\lim _{n \rightarrow \infty} \frac{\left|\left[\mathbf{S}_{n}(\mathrm{spt} \geq t)\right]\right|}{\left|\left[\mathbf{S}_{n}\left(\mathrm{spt}^{*} \geq m\right)\right]\right|}=0 .
$$

(ii) There is $T>m$ such that

$$
\lim _{n \rightarrow \infty} \frac{\left|\left[\mathbf{S}_{n}(\mathrm{spt} \geq m) \cap \mathbf{S}_{n}\left(\mathrm{spt}^{*} \leq T\right)\right]\right|}{\left|\left[\mathbf{S}_{n}(\mathrm{spt} \geq m)\right]\right|}=\lim _{n \rightarrow \infty} \frac{\left|\left[\mathbf{S}_{n}\left(\mathrm{spt}^{*} \geq m\right) \cap \mathbf{S}_{n}\left(\mathrm{spt}^{*} \leq T\right)\right]\right|}{\left|\left[\mathbf{S}_{n}\left(\mathrm{spt}^{*} \geq m\right)\right]\right|}=1
$$


Proof Part (i) follows immediately from Proposition 7.4, because if $f \in S y m_{n}$ and $\operatorname{spt}(f)=m$, then $\mathbf{S}_{n}(f) \subseteq \mathbf{S}_{n}(\mathrm{spt} \geq m) \subseteq \mathbf{S}_{n}\left(\mathrm{spt}^{*} \geq m\right)$.

Part (ii) is proved like Corollary 3.7, but with part (i) instead of Corollary 2.5.

Corollary 7.6 For every finite group $G$ there is $T \in \mathbb{N}$ such that

$$
\lim _{n \rightarrow \infty} \frac{\mid\left[\left\{\mathcal{M} \in \mathbf{S}_{n}: G \leq \operatorname{Aut}(\mathcal{M}) \text { and } \operatorname{spt}^{*}(\mathcal{M}) \leq T\right\}\right] \mid}{\left|\left[\left\{\mathcal{M} \in \mathbf{S}_{n}: G \leq \operatorname{Aut}(\mathcal{M})\right\}\right]\right|}=1
$$

Proof Let $G$ be isomorphic to a permutation group without fixed points on $[\mathrm{m}]$ for some $m \in \mathbb{N}^{+}$. Let $t=2 r(m !-1) m / m !+1$. In the same way as we proved Corollary 2.6, but using Proposition 7.4 instead of Proposition 2.3, we get

$$
\lim _{n \rightarrow \infty} \frac{\left.\mid\left[\left\{\mathcal{M} \in \mathbf{S}_{n}: G \leq \operatorname{Aut}(\mathcal{M}) \text { and } \operatorname{spt}(\mathcal{M}) \leq t\right)\right\}\right] \mid}{\left|\left[\left\{\mathcal{M} \in \mathbf{S}_{n}: G \leq \operatorname{Aut}(\mathcal{M})\right\}\right]\right|}=1 .
$$

By Proposition 3.5 the sought after $T$ exists.

Theorem 7.7 For each result in the previous sections which, for some sequence $\mathbf{S}_{n}^{\prime} \subseteq \mathbf{S}_{n}, n \in \mathbb{N}^{+}$, and set $\mathbf{X} \subseteq \mathbf{S}$ that is closed under isomorphism, can be stated in the form

$$
\lim _{n \rightarrow \infty} \frac{\left|\mathbf{S}_{n}^{\prime} \cap \mathbf{X}\right|}{\left|\mathbf{S}_{n}^{\prime}\right|}=c
$$

where $0 \leq c \leq 1$, we also have

$$
\lim _{n \rightarrow \infty} \frac{\left|\left[\mathbf{S}_{n}^{\prime} \cap \mathbf{X}\right]\right|}{\left|\left[\mathbf{S}_{n}^{\prime}\right]\right|}=c
$$

for the same constant $c$.

Remark 7.8 The statement in Theorem 7.7 that we get exactly the same limit $c$ in both the labelled and unlabelled case may seem counter intuitive, because we consider structures with a nontrivial automorphism. Roughly speaking, the reason why we indeed get exactly the same limit in the labelled and the unlabelled case is that for each $\mathbf{S}^{\prime}=\bigcup_{n \in \mathbb{N}^{+}} \mathbf{S}_{n}^{\prime}$ considered, there is $p$ such that $\left|\mathbf{S}_{n}^{\prime}\right| \sim\left|\mathbf{S}_{n}^{\prime} \cap \mathbf{S}_{n}\left(\operatorname{spt}^{*} \leq p\right)\right|$ and therefore Lemma 7.3 can be applied in the proof of Theorem 7.7.

Example 7.9 Here are two examples of applications of Theorem 7.7.

(i) Let $t \geq 2$, let $\varphi$ be a sentence and let

$$
\mathbf{X}_{\varphi}=\{\mathcal{M} \in \mathbf{S}: \mathcal{M}=\varphi\} .
$$

Journal of Logic \& Analysis 7:2 (2015) 
By Theorem 6.2, $\left|\mathbf{S}_{n}(\mathrm{spt} \geq t) \cap \mathbf{X}_{\varphi}\right| /\left|\mathbf{S}_{n}(\mathrm{spt} \geq t)\right|$ converges to some $0 \leq c \leq 1$ as $n \rightarrow \infty$. Now Theorem 7.7 implies that

$$
\lim _{n \rightarrow \infty} \frac{\left|\left[\mathbf{S}_{n}(\mathrm{spt} \geq t) \cap \mathbf{X}_{\varphi}\right]\right|}{\left|\left[\mathbf{S}_{n}(\mathrm{spt} \geq t)\right]\right|}=c .
$$

(ii) Let $G$ be a finite group, $\varphi$ a sentence and $\mathbf{X}_{\varphi}$ as above. By Theorem 6.2,

$$
\frac{\left|\left\{\mathcal{M} \in \mathbf{S}_{n}: G \leq \operatorname{Aut}(\mathcal{M})\right\} \cap \mathbf{X}_{\varphi}\right|}{\left|\left\{\mathcal{M} \in \mathbf{S}_{n}: G \leq \operatorname{Aut}(\mathcal{M})\right\}\right|}
$$

converges to some $0 \leq c \leq 1$. Theorem 7.7 implies that

$$
\lim _{n \rightarrow \infty} \frac{\left|\left[\left\{\mathcal{M} \in \mathbf{S}_{n}: G \leq \operatorname{Aut}(\mathcal{M})\right\} \cap \mathbf{X}_{\varphi}\right]\right|}{\left|\left[\left\{\mathcal{M} \in \mathbf{S}_{n}: G \leq \operatorname{Aut}(\mathcal{M})\right\}\right]\right|}=c .
$$

Proof of Theorem 7.7. Suppose that $\mathbf{S}_{n}^{\prime} \subseteq \mathbf{S}_{n}$, for $n \in \mathbb{N}^{+}$, that $\mathbf{X} \subseteq \mathbf{S}$ is closed under isomorphism and that we have proved (in previous sections) that

$$
\lim _{n \rightarrow \infty} \frac{\left|\mathbf{S}_{n}^{\prime} \cap \mathbf{X}\right|}{\left|\mathbf{S}_{n}^{\prime}\right|}=c
$$

for some $0 \leq c \leq 1$. In all of these cases it is clear that $\mathbf{S}^{\prime}=\bigcup_{n=1}^{\infty} \mathbf{S}_{n}^{\prime}$ is closed under isomorphism. It also follows, either by definition or by results that have been proved, that there is an integer $p$ such that

$$
\begin{aligned}
& \lim _{n \rightarrow \infty} \frac{\left|\mathbf{S}_{n}^{\prime} \cap \mathbf{S}_{n}\left(\mathrm{spt}^{*} \leq p\right)\right|}{\left|\mathbf{S}_{n}^{\prime}\right|}=1 \quad \text { and } \\
& \lim _{n \rightarrow \infty} \frac{\left|\left[\mathbf{S}_{n}^{\prime} \cap \mathbf{S}_{n}\left(\mathrm{spt}^{*} \leq p\right)\right]\right|}{\left|\left[\mathbf{S}_{n}^{\prime}\right]\right|}=1 .
\end{aligned}
$$

It follows from (7-8) and (7-9) that

$$
\lim _{n \rightarrow \infty} \frac{\left|\mathbf{S}_{n}\left(\mathrm{spt}^{*} \leq p\right) \cap \mathbf{S}^{\prime} \cap \mathbf{X}\right|}{\left|\mathbf{S}_{n}\left(\mathrm{spt}^{*} \leq p\right) \cap \mathbf{S}^{\prime}\right|}=c .
$$

Lemma 7.3 with $\mathbf{Y}=\mathbf{S}^{\prime}$ gives

$$
\left|\mathbf{S}_{n}\left(\mathrm{spt}^{*} \leq p\right) \cap \mathbf{S}^{\prime}\right| \sim n !\left|\left[\mathbf{S}_{n}\left(\mathrm{spt}^{*} \leq p\right) \cap \mathbf{S}^{\prime}\right]\right|,
$$

and with $\mathbf{Y}=\mathbf{S}^{\prime} \cap \mathbf{X}$ it gives

$$
\left|\mathbf{S}_{n}\left(\mathrm{spt}^{*} \leq p\right) \cap \mathbf{S}^{\prime} \cap \mathbf{X}\right| \sim n !\left|\left[\mathbf{S}_{n}\left(\mathrm{spt}^{*} \leq p\right) \cap \mathbf{S}^{\prime} \cap \mathbf{X}\right]\right| .
$$

This together with $(7-11)$ gives

$$
\begin{aligned}
\frac{\left|\left[\mathbf{S}_{n}\left(\mathrm{spt}^{*} \leq p\right) \cap \mathbf{S}^{\prime} \cap \mathbf{X}\right]\right|}{\left|\left[\mathbf{S}_{n}\left(\mathrm{spt}^{*} \leq p\right) \cap \mathbf{S}^{\prime}\right]\right|} & =\frac{n !\left|\left[\mathbf{S}_{n}\left(\mathrm{spt}^{*} \leq p\right) \cap \mathbf{S}^{\prime} \cap \mathbf{X}\right]\right|}{n !\left|\left[\mathbf{S}_{n}\left(\mathrm{spt}^{*} \leq p\right) \cap \mathbf{S}^{\prime}\right]\right|} \\
& \sim \frac{\left|\mathbf{S}_{n}\left(\mathrm{spt}^{*} \leq p\right) \cap \mathbf{S}^{\prime} \cap \mathbf{X}\right|}{\left|\mathbf{S}_{n}\left(\mathrm{spt}^{*} \leq p\right) \cap \mathbf{S}^{\prime}\right|} \rightarrow c \quad \text { as } n \rightarrow \infty .
\end{aligned}
$$


Combining this with (7-10) gives

$$
\text { (7-12) } \begin{aligned}
& \frac{\left|\left[\mathbf{S}_{n}\left(\mathrm{spt}^{*} \leq p\right) \cap \mathbf{S}_{n}^{\prime} \cap \mathbf{X}\right]\right|}{\left|\left[\mathbf{S}_{n}^{\prime}\right]\right|} \\
= & \frac{\left|\left[\mathbf{S}_{n}\left(\mathrm{spt}^{*} \leq p\right) \cap \mathbf{S}_{n}^{\prime} \cap \mathbf{X}\right]\right|}{\left|\left[\mathbf{S}_{n}\left(\mathrm{spt}^{*} \leq p\right) \cap \mathbf{S}_{n}^{\prime}\right]\right|} \cdot \frac{\left|\left[\mathbf{S}_{n}\left(\mathrm{spt}^{*} \leq p\right) \cap \mathbf{S}_{n}^{\prime}\right]\right|}{\left|\left[\mathbf{S}_{n}^{\prime}\right]\right|} \rightarrow c \quad \text { as } n \rightarrow \infty .
\end{aligned}
$$

Finally we have

$$
\frac{\left|\left[\mathbf{S}_{n}^{\prime} \cap \mathbf{X}\right]\right|}{\left|\left[\mathbf{S}_{n}^{\prime}\right]\right|}=\frac{\left|\left[\mathbf{S}_{n}\left(\mathrm{spt}^{*} \leq p\right) \cap \mathbf{S}_{n}^{\prime} \cap \mathbf{X}\right]\right|}{\left|\left[\mathbf{S}_{n}^{\prime}\right]\right|}+\frac{\left|\left[\mathbf{S}_{n}\left(\mathrm{spt}^{*} \geq p+1\right) \cap \mathbf{S}_{n}^{\prime} \cap \mathbf{X}\right]\right|}{\left|\left[\mathbf{S}_{n}^{\prime}\right]\right|}
$$

which tends to $c$ as $n \rightarrow \infty$, because of (7-12) and (7-10). This concludes the proof of Theorem 7.7.

\section{References}

[1] W Burnside, On the Theory of Groups of Finite Order, Proc. London Math. Soc. S2-7 (1897) 1-c

[2] P J Cameron, On graphs with given automorphism group, European J. Combin. 1 (1980) 91-96

[3] P J Cameron, On graphs with given automorphism group, from: "Topics in algebraic graph theory", (L W Beineke, R J Wilson, editors), Encyclopedia of Mathematics and its Applications 102, Cambridge University Press, Cambridge (2004) xvi+276

[4] K J Compton, The computational complexity of asymptotic problems. I. Partial orders, Inform. and Comput. 78 (1988) 108-123

[5] JD Dixon, B Mortimer, Permutation groups, volume 163 of Graduate Texts in Mathematics, Springer-Verlag, New York (1996)

[6] H-D Ebbinghaus, J Flum, Finite model theory, Perspectives in Mathematical Logic, Springer-Verlag, Berlin (1995)

[7] P Erdős, A Rényi, Asymmetric graphs, Acta Math. Acad. Sci. Hungar 14 (1963) 295-315

[8] R Fagin, Probabilities on finite models, J. Symbolic Logic 41 (1976) 50-58

[9] R Fagin, The number of finite relational structures, Discrete Math. 19 (1977) 17-21

[10] G W Ford, G E Uhlenbeck, Combinatorial problems in the theory of graphs. IV, Proc. Nat. Acad. Sci. U.S.A. 43 (1957) 163-167

[11] J V Glebskii, D I Kogan, M I Liogonkii, V A Talanov, Volume and fraction of satisfiability of formulas of the lower predicate calculus, Kibernetika (Kiev) (1969) $17-27$ 
[12] F Harary, Note on Carnap's relational asymptotic relative frequencies, J. Symb. Logic 23 (1958) 257-260

[13] D J Kleitman, B L Rothschild, Asymptotic enumeration of partial orders on a finite set, Trans. Amer. Math. Soc. 205 (1975) 205-220

[14] P G Kolaitis, H J Prömel, B L Rothschild, $K_{l+1}$-free graphs: asymptotic structure and a 0-1 law, Trans. Amer. Math. Soc. 303 (1987) 637-671

[15] V Koponen, Asymptotic probabilities of extension properties and random l-colourable structures, Ann. Pure Appl. Logic 163 (2012) 391-438

[16] V Koponen, Typical automorphism groups of finite nonrigid structures, Archive for Mathematical Logic (provisionally accepted for publication)

[17] D Marker, Model theory, volume 217 of Graduate Texts in Mathematics, SpringerVerlag, New York (2002)

[18] W Oberschelp, Strukturzahlen in endlichen Relationssystemen, from: "Contributions to Math. Logic (Colloquium, Hannover, 1966)", North-Holland, Amsterdam (1968) 199-213

[19] P Rothmaler, Introduction to model theory, volume 15 of Algebra, Logic and Applications, Gordon and Breach Science Publishers, Amsterdam (2000)

Department of Mathematics, Uppsala University, Box 480, 75106 Uppsala, Sweden

Department of Mathematics, Uppsala University, Box 480, 75106 Uppsala, Sweden

ove@math.uu.se, vera@math.uu.se

Received: 16 November $2013 \quad$ Revised: 19 March 2015 